- Supporting Information -

\title{
A Strategy for the Construction of Triply Interlocked Organometallic Cages by Rational Design of Poly-NHC Precursors
}

Ya-Wen Zhang, Sha Bai, Yao-Yu Wang, and Ying-Feng Han*

Key Laboratory of Synthetic and Natural Functional Molecule of the Ministry of Education, College of Chemistry and Materials Science, Northwest University, Xi'an 710127, P. R. China 


\section{Table of Contents:}

$\begin{array}{ll}\text { 1. Materials and methods } & \text { S2 }\end{array}$

2. Synthesis of imidazo[1,5-a]pyridine-based N-heterocyclic carbene precursors S2

3. Synthesis of compounds $\left[\mathrm{Ag}_{3}(\mathbf{L})_{2}\right]_{2}\left(\mathrm{PF}_{6}\right)_{6}(\mathbf{L}=\mathbf{1 a}-\mathbf{1 c}) \quad \mathrm{S} 16$

4. Synthesis of compounds $\left[\mathrm{Au}_{3}(\mathbf{L})_{2}\right]_{2}\left(\mathrm{PF}_{6}\right)_{6}(\mathbf{L}=\mathbf{1 b}, \mathbf{1 c}) \quad$ S31

5. UV-vis absorption spectrum of $\mathrm{H}_{3}-\mathbf{L}\left(\mathrm{PF}_{6}\right)_{3},\left[\mathrm{Ag}_{3}(\mathbf{L})_{2}\right]_{2}\left(\mathrm{PF}_{6}\right)_{6}(\mathbf{L}=\mathbf{1 a}-\mathbf{1 c})$ and $\left[\mathrm{Au}_{3}(\mathbf{L})_{2}\right]_{2}\left(\mathrm{PF}_{6}\right)_{6}(\mathbf{L}=\mathbf{1 b}, \mathbf{1 c}) \quad$ S39

6. Stability of Triply Interlocked $\left[\mathrm{M}_{3}(\mathbf{L})_{2}\right]_{2}(\mathrm{M}=\mathrm{Ag}, \mathrm{Au})$ Cages $\quad \mathrm{S} 40$

7. X-ray diffraction analysis of $\left[\mathrm{Ag}_{3}(\mathbf{1 a})_{2}\right]_{2}\left(\mathrm{PF}_{6}\right)_{6} \quad \mathrm{~S} 50$

8. References $\quad$ S53 


\section{Materials and methods}

All starting materials were used as received from commercial sources unless otherwise stated, while solvents were freshly distilled by standard procedures prior to use. The experiments were carried out under nitrogen atmosphere with standard Schlenk techniques. Compound [2,4,6-tris[4-(4,4,5,5-tetramethyl-1,3,2-dioxaborolan-2yl)phenyl]-1,3,5-triazine] was synthesized according to reported procedure. ${ }^{[\mathrm{S} 1]}$ The ${ }^{1} \mathrm{H}$, ${ }^{13} \mathrm{C}\left\{{ }^{1} \mathrm{H}\right\}$ and 2D NMR spectra were recorded on Bruker AVANCE III 400 or AVANCE III 600 spectrometers. Chemical shifts for ${ }^{1} \mathrm{H},{ }^{13} \mathrm{C}\left\{{ }^{1} \mathrm{H}\right\}$ and $2 \mathrm{D}$ NMR were reported in ppm on the $\delta$ scale; ${ }^{1} \mathrm{H}$ and ${ }^{13} \mathrm{C}\left\{{ }^{1} \mathrm{H}\right\}$ were referenced to the solvent residual peak. Coupling constants $(J)$ are reported in hertz $(\mathrm{Hz})$. The following abbreviations are used to describe signal multiplicity for ${ }^{1} \mathrm{H}$, and ${ }^{13} \mathrm{C}\left\{{ }^{1} \mathrm{H}\right\}$ NMR spectra: s: singlet, d: doublet, t: triplet, m: multiplet, br: broad. Mass spectra were obtained with a Bruker microTOFQ II mass spectrometer (Bruker Daltonics Corp., USA) or a Synapt G2 ESI-Q-TOF mass spectrometer in the electrospray ionization (ESI) mode. The UV-Vis experiments were conducted on an Agilent Cary-100 spectrophotometer. The fluorescence experiments were performed on a Horiba QM8000 spectrometer. 
2. Synthesis and characterization of $\mathrm{N}$-heterocyclic carbene precursors $\mathrm{H}_{3}-\mathrm{L}\left(\mathrm{PF}_{6}\right)_{3}$ (L = 1a-1c)

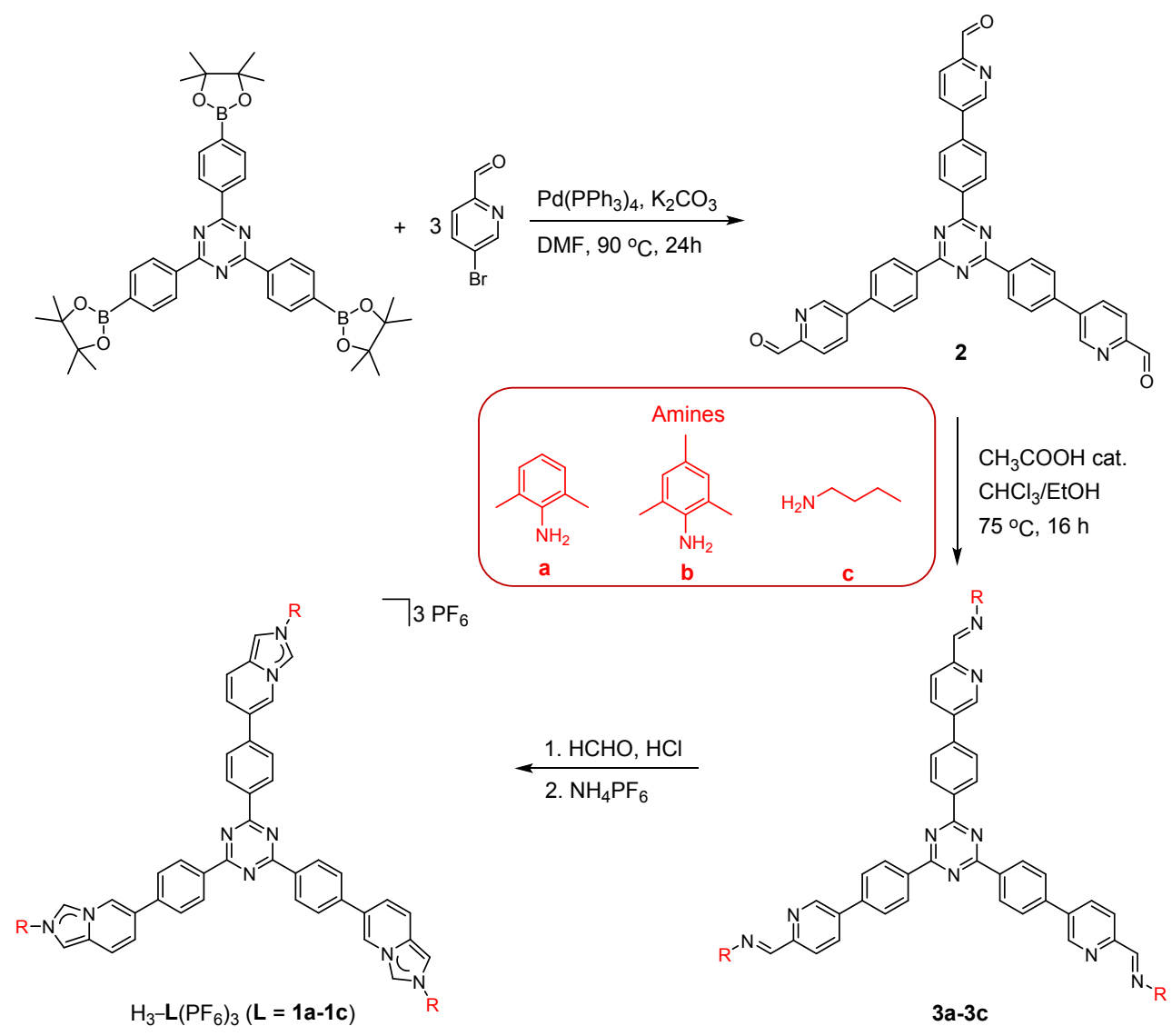

Scheme S1. Synthesis of imidazo[1,5-a]pyridine-based N-heterocyclic carbene precursors $\mathrm{H}_{3}-\mathbf{L}\left(\mathrm{PF}_{6}\right)_{3}(\mathbf{L}=\mathbf{1 a}-\mathbf{1 c})$.

\subsection{Synthesis of compound 2}

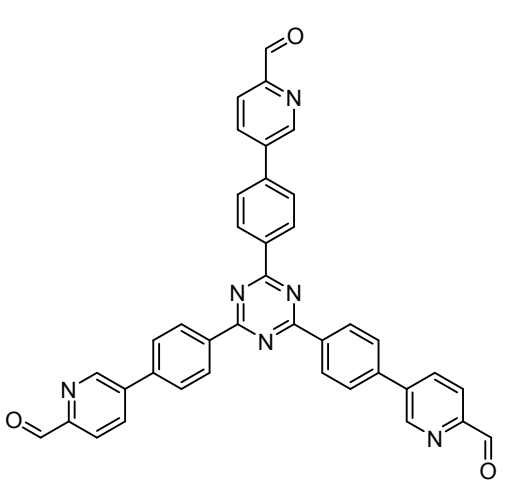

A mixture of 2,4,6-tris[4-(4,4,5,5-tetramethyl-1,3,2dioxaborolan-2-yl)phenyl]-1,3,5-triazine $\quad(500 \mathrm{mg}$, $0.73 \mathrm{mmol}$ ), 5-bromo-2-pyridinecarboxaldehyde (609 $\mathrm{mg}, 3.28 \mathrm{mmol}), \mathrm{K}_{2} \mathrm{CO}_{3}(1610 \mathrm{mg}, 11.68 \mathrm{mmol})$, $\operatorname{Pd}\left(\mathrm{PPh}_{3}\right)_{4}(253 \mathrm{mg}, 0.22 \mathrm{mmol})$ in anhydrous DMF $(80$ $\mathrm{mL}$ ) was degassed and stirred under $\mathrm{N}_{2}$ atmosphere at $90{ }^{\circ} \mathrm{C}$ for $24 \mathrm{~h}$. The solvent was 
removed under reduced pressure and the solid residue triturated with water, collected by filtration and washed with water $(3 \times 30 \mathrm{~mL})$, diethyl ether $(2 \times 10 \mathrm{~mL})$, and hexane $(2 \times 10 \mathrm{~mL})$. The dried solid was washed with a small amount of cold $\mathrm{CH}_{2} \mathrm{Cl}_{2}$ and collected again. The resulting product was re-dissolved in hot $\mathrm{CHCl}_{3}$, the insoluble materials filtered off and $\mathrm{Et}_{2} \mathrm{O}$ added to the filtrate to precipitate out pure product 2 as a pale yellow solid. Yield: $182 \mathrm{mg}(0.292 \mathrm{mmol} 40 \%) .{ }^{1} \mathrm{H}$ NMR $\left(400 \mathrm{MHz}, \mathrm{CDCl}_{3}\right) \delta$ $=10.17(\mathrm{~s}, 3 \mathrm{H}), 9.14(\mathrm{~s}, 3 \mathrm{H}), 8.96(\mathrm{~d}, J=8.3 \mathrm{~Hz}, 6 \mathrm{H}), 8.20(\mathrm{~d}, J=7.9 \mathrm{~Hz}, 3 \mathrm{H}), 8.12$ $(\mathrm{d}, J=8.0 \mathrm{~Hz}, 3 \mathrm{H}), 7.90(\mathrm{~d}, J=8.3 \mathrm{~Hz}, 6 \mathrm{H}) \mathrm{ppm} .{ }^{13} \mathrm{C}\left\{{ }^{1} \mathrm{H}\right\}$ NMR $\left(125 \mathrm{MHz}, \mathrm{CDCl}_{3}\right)$ : $\delta=193.1,171.4,152.2,148.9,140.8,139.9,136.8,135.6,130.1,127.9,122.1 \mathrm{ppm}$. HRMS (ESI, positive ions): $m / z=313.1146$ (calcd for $[2+2 \mathrm{H}]^{2+} 313.1028$ ).

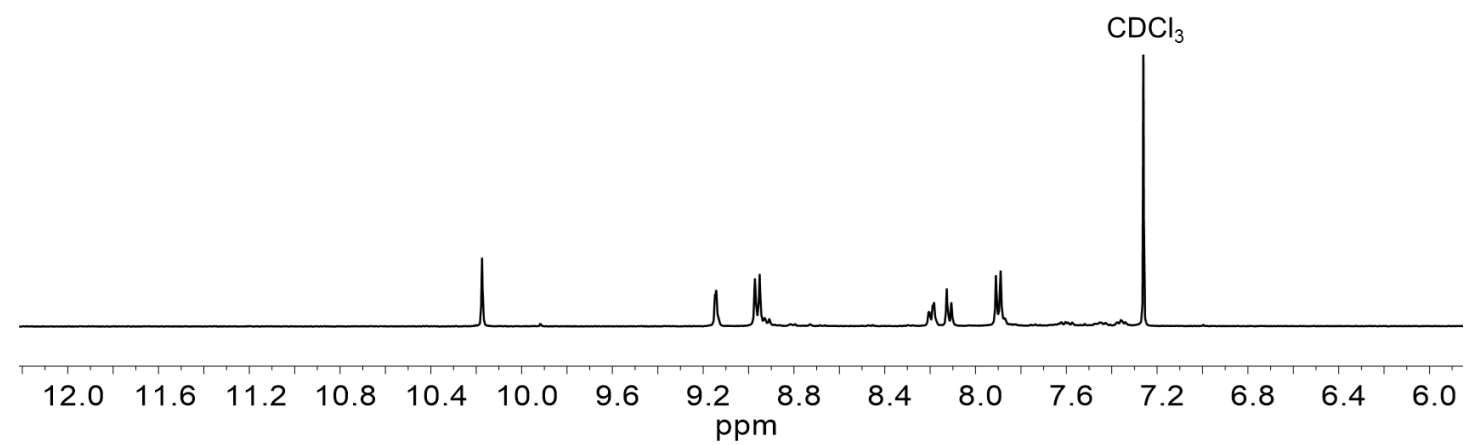

Figure S1. ${ }^{1} \mathrm{H}$ NMR spectrum $\left(400 \mathrm{MHz}, \mathrm{CDCl}_{3}\right)$ of 2.

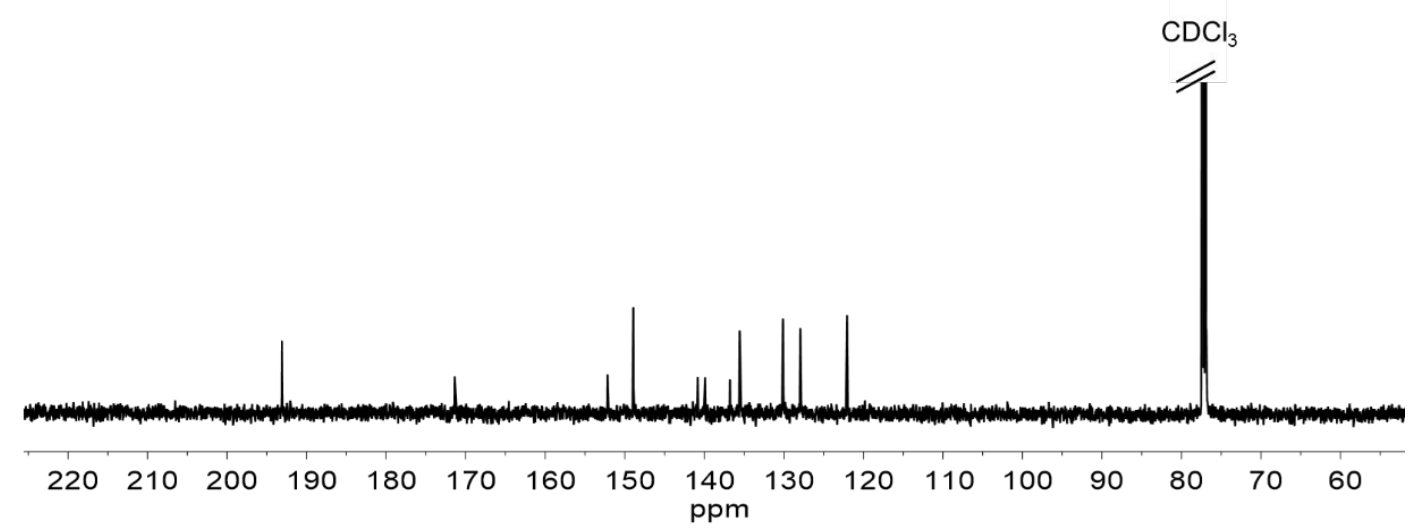

Figure S2. ${ }^{13} \mathrm{C}\left\{{ }^{1} \mathrm{H}\right\}$ NMR spectrum $\left(100 \mathrm{MHz}, \mathrm{CDCl}_{3}\right)$ of 2. 


\subsection{Synthesis of compound 3a-3c}

Compound 5,5',5"-((1,3,5-triazine-2,4,6-triyl)tris(benzene-4,1-diyl))tripicolinaldehyde (2) (200 mg, $0.32 \mathrm{mmol})$ was dissolved in $30 \mathrm{~mL}$ trichloromethane and $10 \mathrm{~mL}$ ethanol, then, $\mathbf{a}$ (b or c) (1.44 mmol) and acetic acid glacial (few drops) was added. The mixture was refluxed for 16 hours. The solvent was removed in vacuo and the residue was washed with methanol yielding 3a (239 mg, 80\%), 3b (265 mg, 85\%) and 3c (197 mg, $78 \%$ ) as a yellow micro-crystalline powder. The samples are unstable, so were used for the next step without further purification.

\subsection{Synthesis of compound $\mathrm{H}_{3}-1 \mathrm{a}\left(\mathrm{PF}_{6}\right)_{3}$}

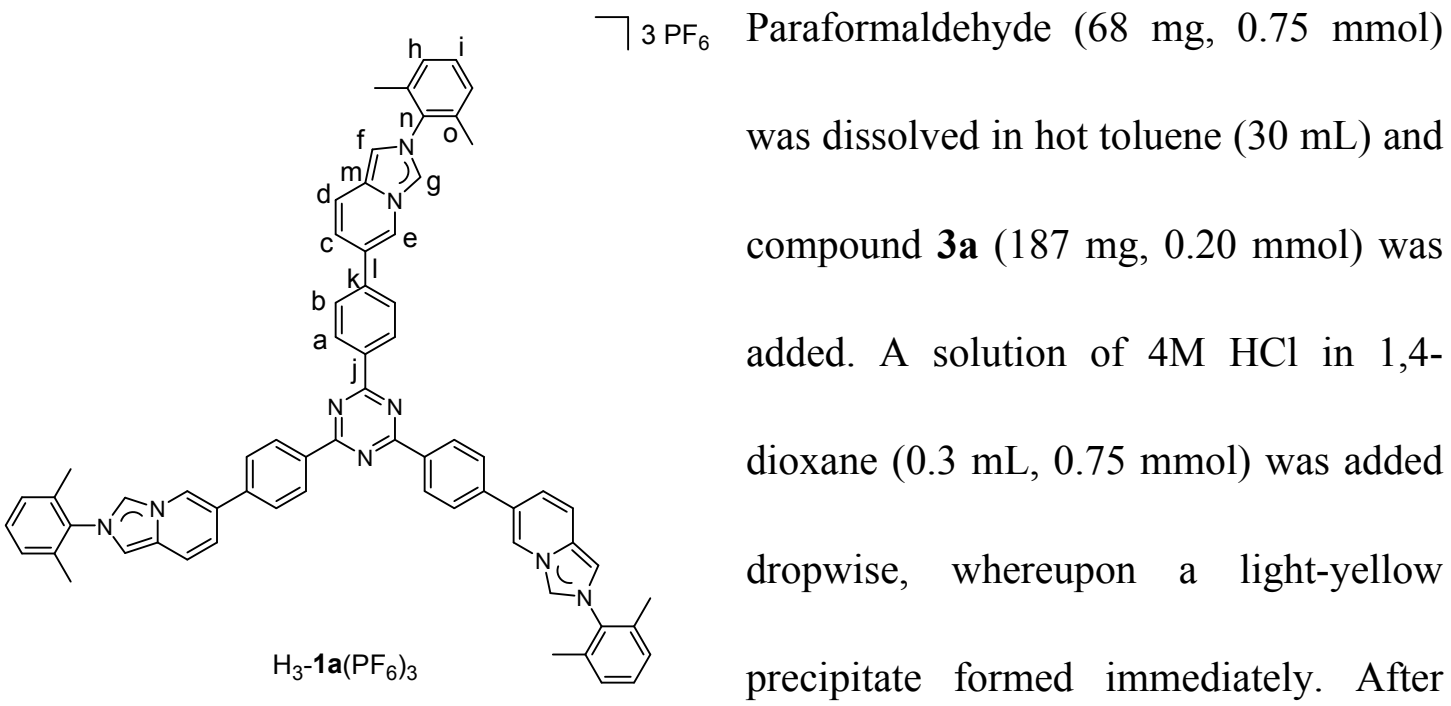

stirring for $18 \mathrm{~h}$ at $43{ }^{\circ} \mathrm{C}$, the precipitate was filtered and washed twice with diethyl ether $(2 \times 2 \mathrm{~mL})$. To remove unreacted paraformaldehyde, the residue was dissolved in methanol and filtered. A solution of $\mathrm{NH}_{4} \mathrm{PF}_{6}$ was then added and the mixture stirred for $8 \mathrm{~h}$ at ambient temperature to precipitate compound $\mathrm{H}_{3}-\mathbf{1} \mathbf{a}\left(\mathrm{PF}_{6}\right)_{3}$ as an off-white solid. Yield: $211 \mathrm{mg}(0.15 \mathrm{mmol}, 75 \%) .{ }^{1} \mathrm{H} \mathrm{NMR}\left(600 \mathrm{MHz}, \mathrm{CD}_{3} \mathrm{CN}\right) \delta=9.25\left(\mathrm{~s}, 3 \mathrm{H}, \mathrm{H}_{\mathrm{g}}\right)$, $9.00\left(\mathrm{~d}, J=8.0 \mathrm{~Hz}, 6 \mathrm{H}, \mathrm{H}_{\mathrm{a}}\right), 8.80\left(\mathrm{~s}, 3 \mathrm{H}, \mathrm{H}_{\mathrm{e}}\right), 8.01\left(\mathrm{~d}, J=8.0 \mathrm{~Hz}, 6 \mathrm{H}, \mathrm{H}_{\mathrm{b}}\right), 7.99-7.95$ 
$\left(\mathrm{m}, 6 \mathrm{H}, \mathrm{H}_{\mathrm{d}}, \mathrm{H}_{\mathrm{f}}\right), 7.79\left(\mathrm{~d}, J=9.2 \mathrm{~Hz}, 3 \mathrm{H}, \mathrm{H}_{\mathrm{c}}\right), 7.51\left(\mathrm{t}, J=7.7 \mathrm{~Hz}, 3 \mathrm{H}, \mathrm{H}_{\mathrm{i}}\right), 7.38(\mathrm{~d}, J=$ $\left.7.7 \mathrm{~Hz}, 6 \mathrm{H}, \mathrm{H}_{\mathrm{h}}\right), 2.12\left(\mathrm{~s}, 18 \mathrm{H}, \mathrm{CH}_{3}\right) \mathrm{ppm} .{ }^{13} \mathrm{C}\left\{{ }^{1} \mathrm{H}\right\} \mathrm{NMR}\left(150 \mathrm{MHz}, \mathrm{CD}_{3} \mathrm{CN}\right) \delta=172.2$ $\left(\mathrm{C}_{\text {triazine }}\right), 140.4\left(\mathrm{C}_{\mathrm{k}}\right), 137.7\left(\mathrm{C}_{\mathrm{j}}\right), 136.0\left(\mathrm{C}_{\mathrm{o}}\right), 134.9\left(\mathrm{C}_{\mathrm{n}}\right), 132.3\left(\mathrm{C}_{\mathrm{i}}\right), 131.9\left(\mathrm{C}_{\mathrm{l}}\right), 131.0\left(\mathrm{C}_{\mathrm{m}}\right)$, $130.9\left(\mathrm{C}_{\mathrm{a}}\right), 129.9\left(\mathrm{C}_{\mathrm{h}}\right), 128.8\left(\mathrm{C}_{\mathrm{b}}\right), 127.6\left(\mathrm{C}_{\mathrm{g}}\right), 127.1\left(\mathrm{C}_{\mathrm{c}}\right), 122.8\left(\mathrm{C}_{\mathrm{e}}\right), 119.8\left(\mathrm{C}_{\mathrm{d}}\right)$, 115.8 $\left(\mathrm{C}_{\mathrm{f}}\right), 17.5\left(\mathrm{CH}_{3}\right) \mathrm{ppm}$. HRMS (ESI, positive ions): $\mathrm{m} / z=558.7038$ (calcd for $\left[\mathrm{H}_{3}-\right.$ $\left.\mathbf{1 a}\left(\mathrm{PF}_{6}\right)\right]^{2+}$ 558.7067), 324.1494 (calcd for $\left.\left[\mathrm{H}_{3}-\mathbf{1 a}\right]^{3+} 324.1495\right)$. 


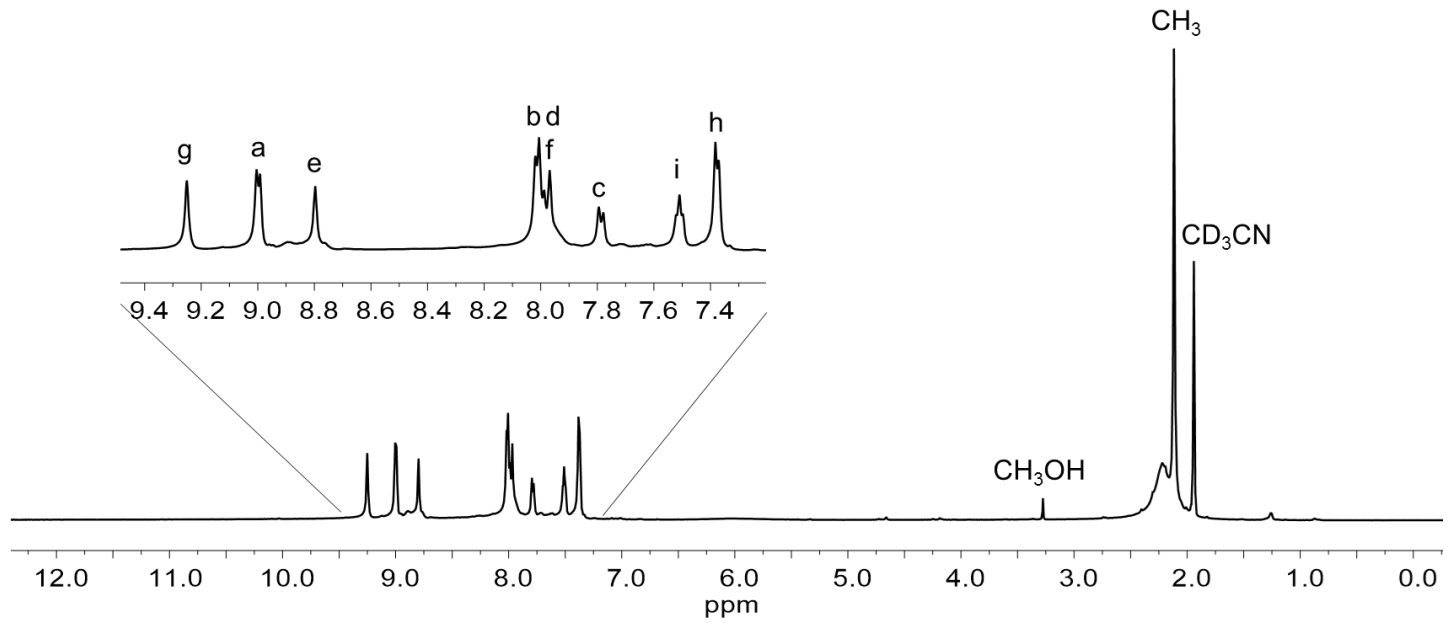

Figure S3. ${ }^{1} \mathrm{H}$ NMR spectrum $\left(600 \mathrm{MHz}, \mathrm{CD}_{3} \mathrm{CN}\right)$ of $\mathrm{H}_{3}-\mathbf{1} \mathbf{a}\left(\mathrm{PF}_{6}\right)_{3}$.

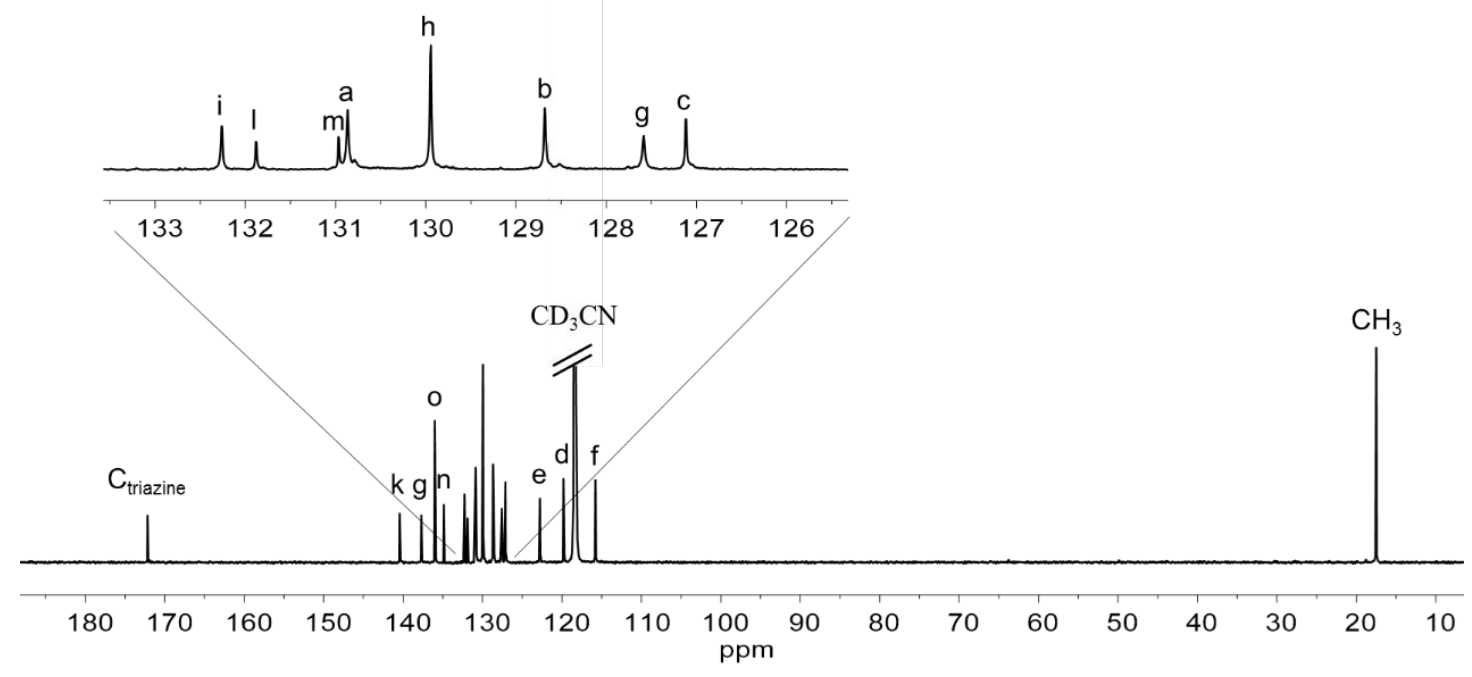

Figure S4. ${ }^{13} \mathrm{C}\left\{{ }^{1} \mathrm{H}\right\}$ NMR spectrum $\left(150 \mathrm{MHz}, \mathrm{CD}_{3} \mathrm{CN}\right)$ of $\mathrm{H}_{3}-\mathbf{1} \mathbf{a}\left(\mathrm{PF}_{6}\right)_{3}$. 


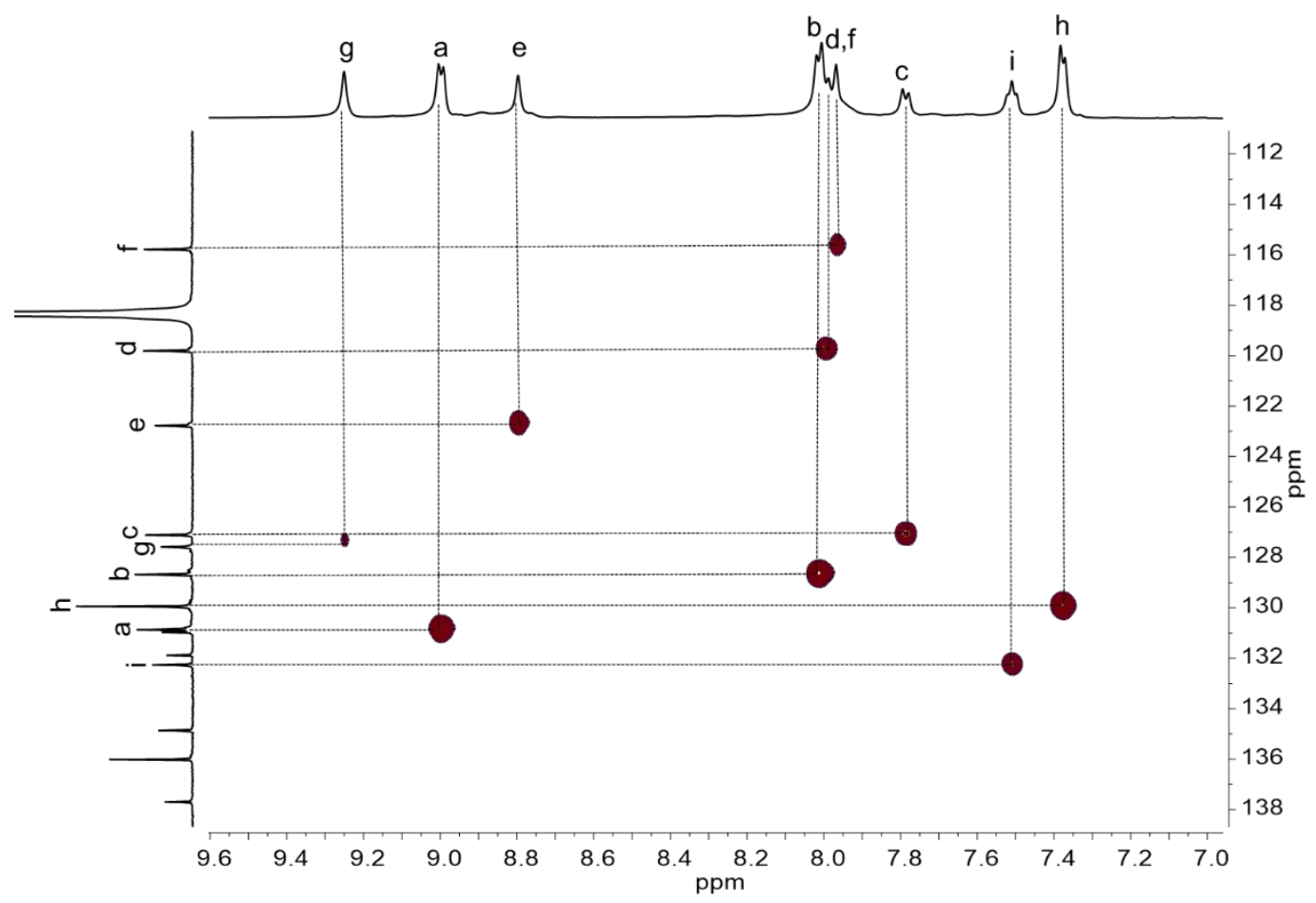

Figure S5. ${ }^{1} \mathrm{H}-{ }^{13} \mathrm{C}$ HSQC spectrum $\left(600 \mathrm{MHz}, \mathrm{CD}_{3} \mathrm{CN}\right)$ of $\mathrm{H}_{3}-\mathbf{1 a}\left(\mathrm{PF}_{6}\right)_{3}$.

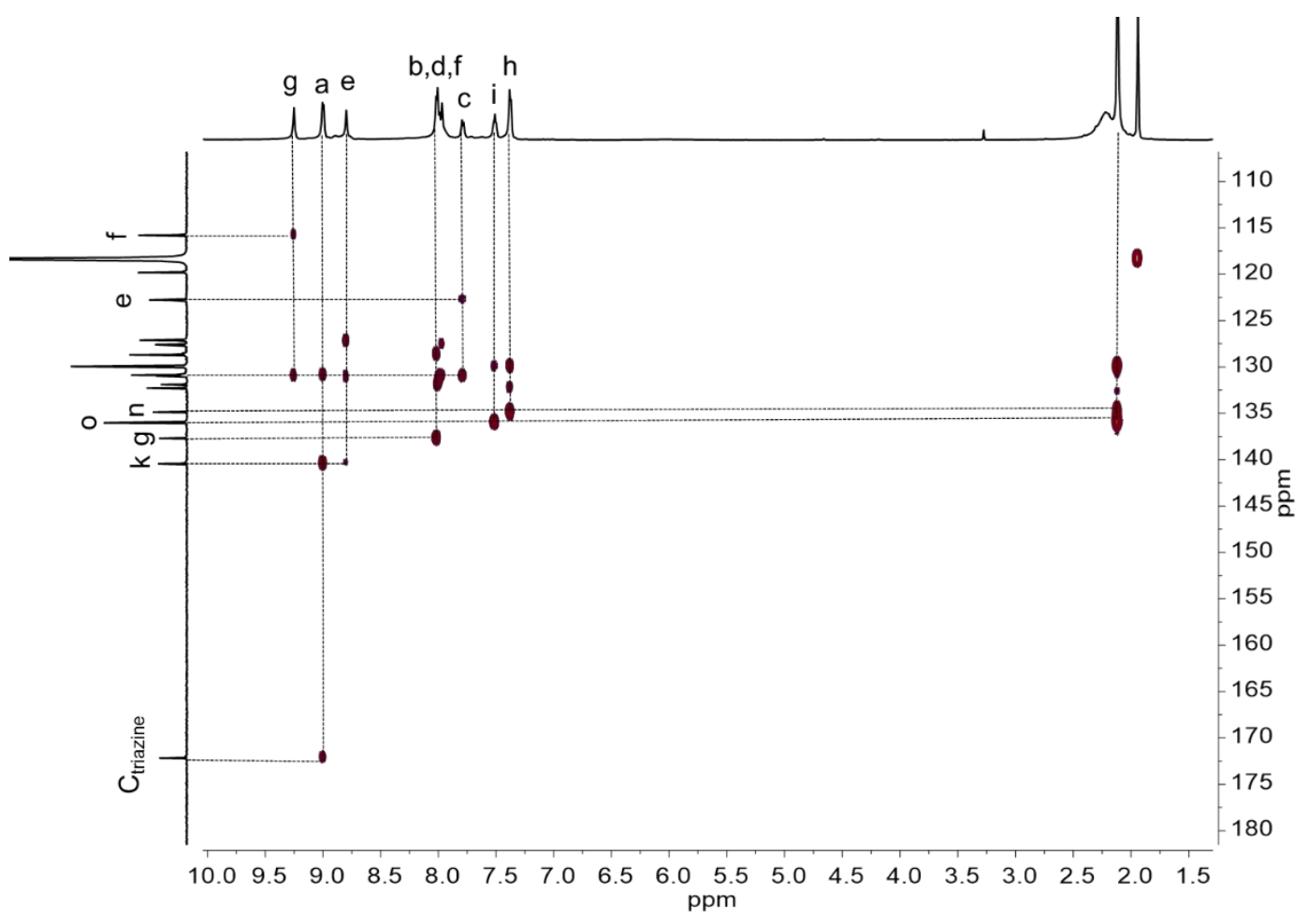

Figure S6. ${ }^{1} \mathrm{H}^{13} \mathrm{C} \mathrm{HMBC}$ spectrum $\left(600 \mathrm{MHz}, \mathrm{CD}_{3} \mathrm{CN}\right)$ of $\mathrm{H}_{3}-\mathbf{1 a}\left(\mathrm{PF}_{6}\right)_{3}$. 

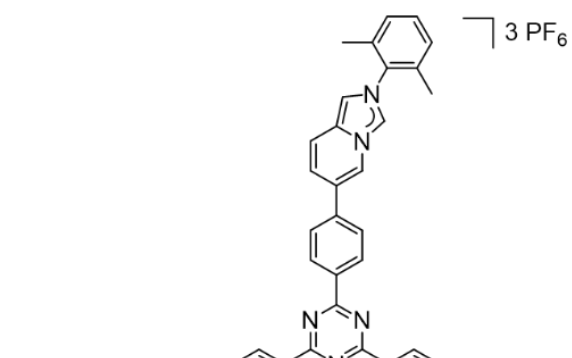

$+3$
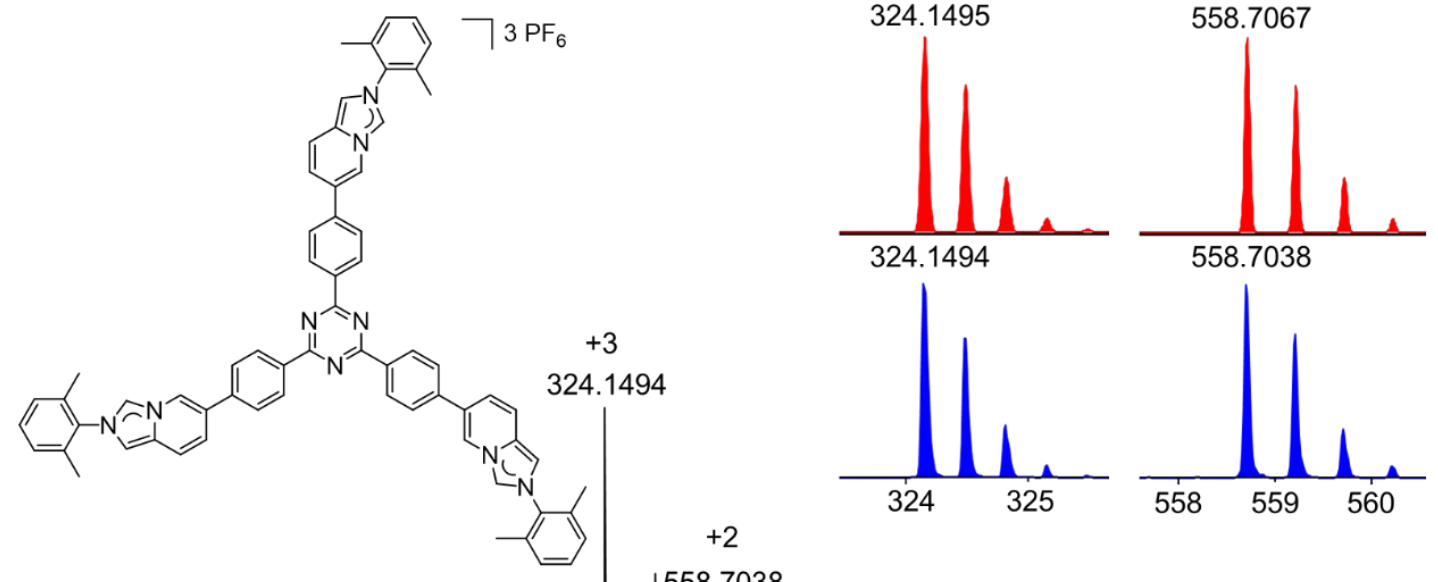

558.7038

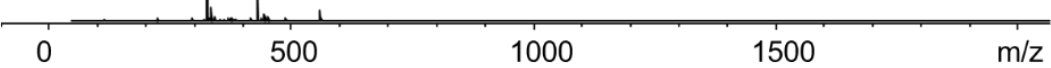

Figure S7. HR-ESI mass spectrum of $\mathrm{H}_{3}-\mathbf{1 a}\left(\mathrm{PF}_{6}\right)_{3}$ with isotope distribution for selected peaks (experimental in blue, calculated in red, $\mathrm{PF}_{6}$ - counterions).

\subsection{Synthesis of compound $\mathrm{H}_{3}-1 \mathrm{~b}\left(\mathrm{PF}_{6}\right)_{3}$}

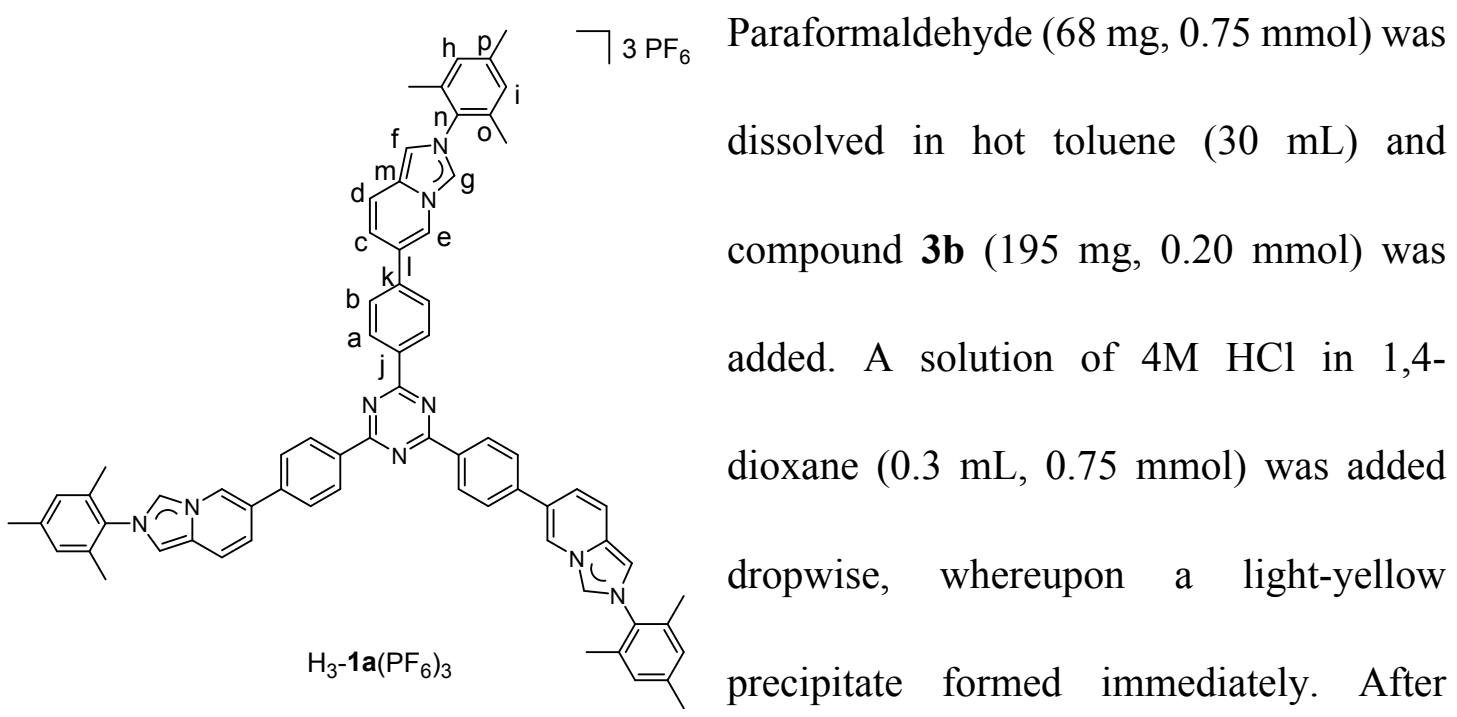

stirring for $18 \mathrm{~h}$ at $43{ }^{\circ} \mathrm{C}$, the precipitate was filtered and washed twice with diethyl ether $(2 \times 2 \mathrm{~mL})$. To remove unreacted paraformaldehyde, the residue was dissolved in methanol and filtered. A solution of $\mathrm{NH}_{4} \mathrm{PF}_{6}$ was then added and the mixture stirred for $8 \mathrm{~h}$ at ambient temperature to precipitate compound $\mathrm{H}_{3}-\mathbf{1 b}\left(\mathrm{PF}_{6}\right)_{3}$ as an off-white solid. Yield: $232 \mathrm{mg}(0.16 \mathrm{mmol}, 80 \%) .{ }^{1} \mathrm{H} \mathrm{NMR}\left(400 \mathrm{MHz}, \mathrm{CD}_{3} \mathrm{CN}\right) \delta=9.20\left(\mathrm{~s}, 3 \mathrm{H}, \mathrm{H}_{\mathrm{g}}\right)$, 
$8.86\left(\mathrm{~d}, J=8.0 \mathrm{~Hz}, 6 \mathrm{H}, \mathrm{H}_{\mathrm{a}}\right), 8.73\left(\mathrm{~s}, 3 \mathrm{H}, \mathrm{H}_{\mathrm{e}}\right), 7.92-7.90\left(\mathrm{~m}, 12 \mathrm{H}, \mathrm{H}_{\mathrm{f}}, \mathrm{H}_{\mathrm{d}}, \mathrm{H}_{\mathrm{b}}\right), 7.69(\mathrm{~d}$, $\left.J=9.2 \mathrm{~Hz}, 3 \mathrm{H}, \mathrm{H}_{\mathrm{c}}\right), 7.18\left(\mathrm{~s}, 6 \mathrm{H}, \mathrm{H}_{\mathrm{h}}\right), 2.40(\mathrm{~s}, 9 \mathrm{H}, \mathrm{CH}), 2.06\left(\mathrm{~s}, 18 \mathrm{H}, \mathrm{CH}_{3}\right) \mathrm{ppm} .{ }^{13} \mathrm{C}\left\{{ }^{1} \mathrm{H}\right\}$ NMR $\left(150 \mathrm{MHz}, \mathrm{CD}_{3} \mathrm{CN}\right) \delta=171.8\left(\mathrm{C}_{\text {triazine }}\right), 142.6\left(\mathrm{C}_{\mathrm{p}}\right), 140.0\left(\mathrm{C}_{\mathrm{k}}\right), 137.5\left(\mathrm{C}_{\mathrm{j}}\right), 135.6$ $\left(\mathrm{C}_{\mathrm{o}}\right), 132.4\left(\mathrm{C}_{\mathrm{n}}\right), 131.7\left(\mathrm{C}_{\mathrm{c}}\right), 130.8\left(\mathrm{C}_{\mathrm{m}}\right), 130.7\left(\mathrm{C}_{\mathrm{a}}\right), 130.5\left(\mathrm{C}_{\mathrm{h}, \mathrm{i}}\right), 128.3\left(\mathrm{C}_{\mathrm{b}}\right), 127.8\left(\mathrm{C}_{\mathrm{g}}\right)$, $126.9\left(\mathrm{C}_{\mathrm{c}}\right), 122.6\left(\mathrm{C}_{\mathrm{e}}\right), 119.6\left(\mathrm{C}_{\mathrm{d}}\right), 116.0\left(\mathrm{C}_{\mathrm{f}}\right), 21.2\left(\mathrm{CH}_{3}\right), 17.5\left(\mathrm{CH}_{3}\right)$ ppm. HRMS (ESI, positive ions): $m / z=579.7278$ (calcd for $\left[\mathrm{H}_{3}-\mathbf{1 b}\left(\mathrm{PF}_{6}\right)\right]^{2+}$ 579.7301).

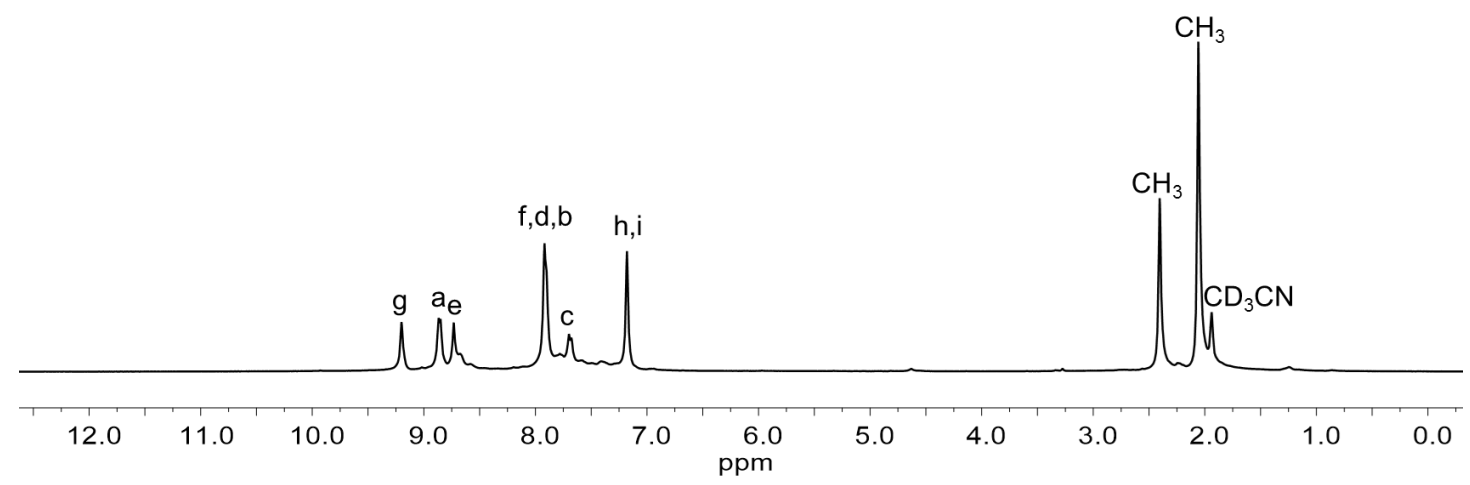

Figure S8. ${ }^{1} \mathrm{H}$ NMR spectrum $\left(600 \mathrm{MHz}, \mathrm{CD}_{3} \mathrm{CN}\right)$ of $\mathrm{H}_{3}-\mathbf{1 b}\left(\mathrm{PF}_{6}\right)_{3}$.

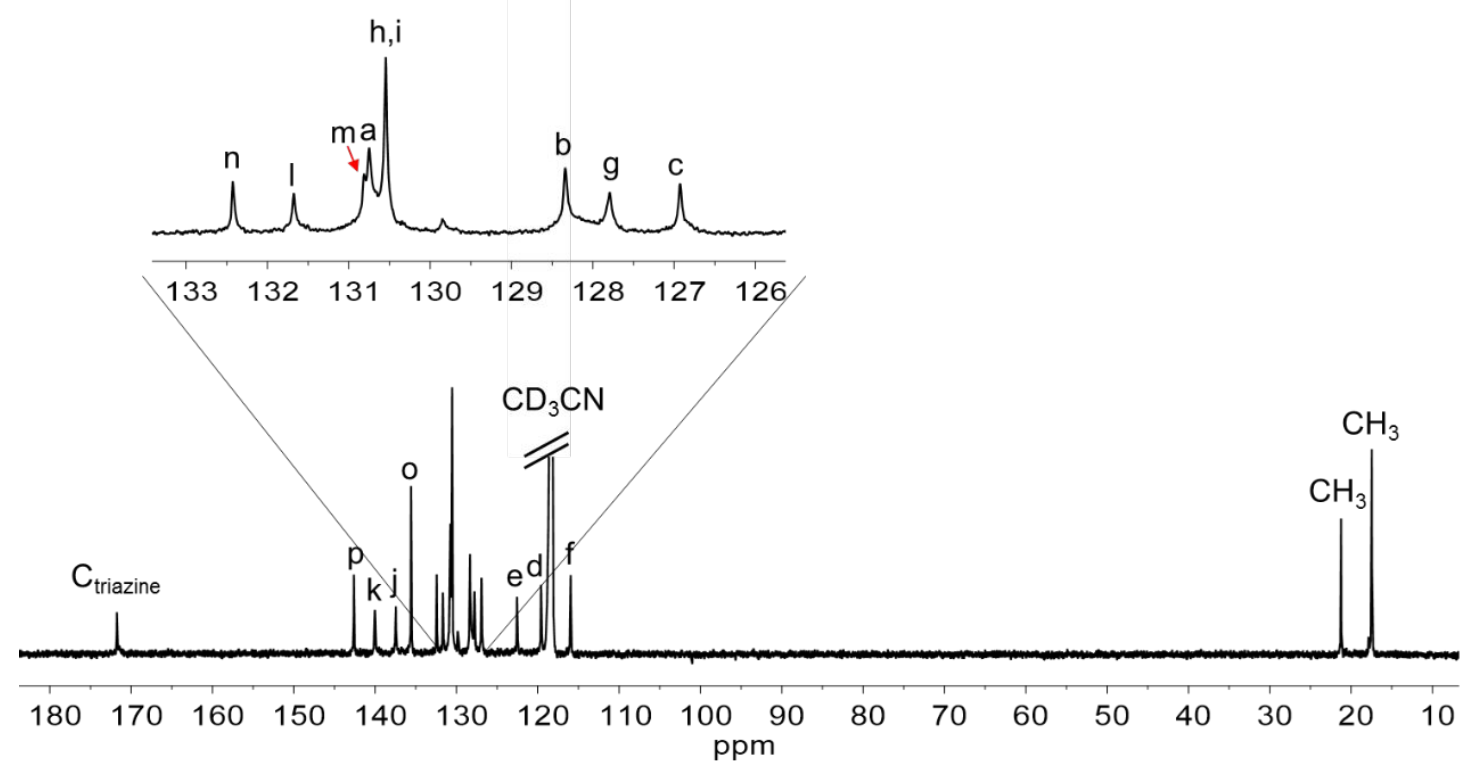

Figure S9. ${ }^{13} \mathrm{C}\left\{{ }^{1} \mathrm{H}\right\}$ spectrum $\left(150 \mathrm{MHz}, \mathrm{CD}_{3} \mathrm{CN}\right)$ of $\mathrm{H}_{3}-\mathbf{1 b}\left(\mathrm{PF}_{6}\right)_{3}$. 


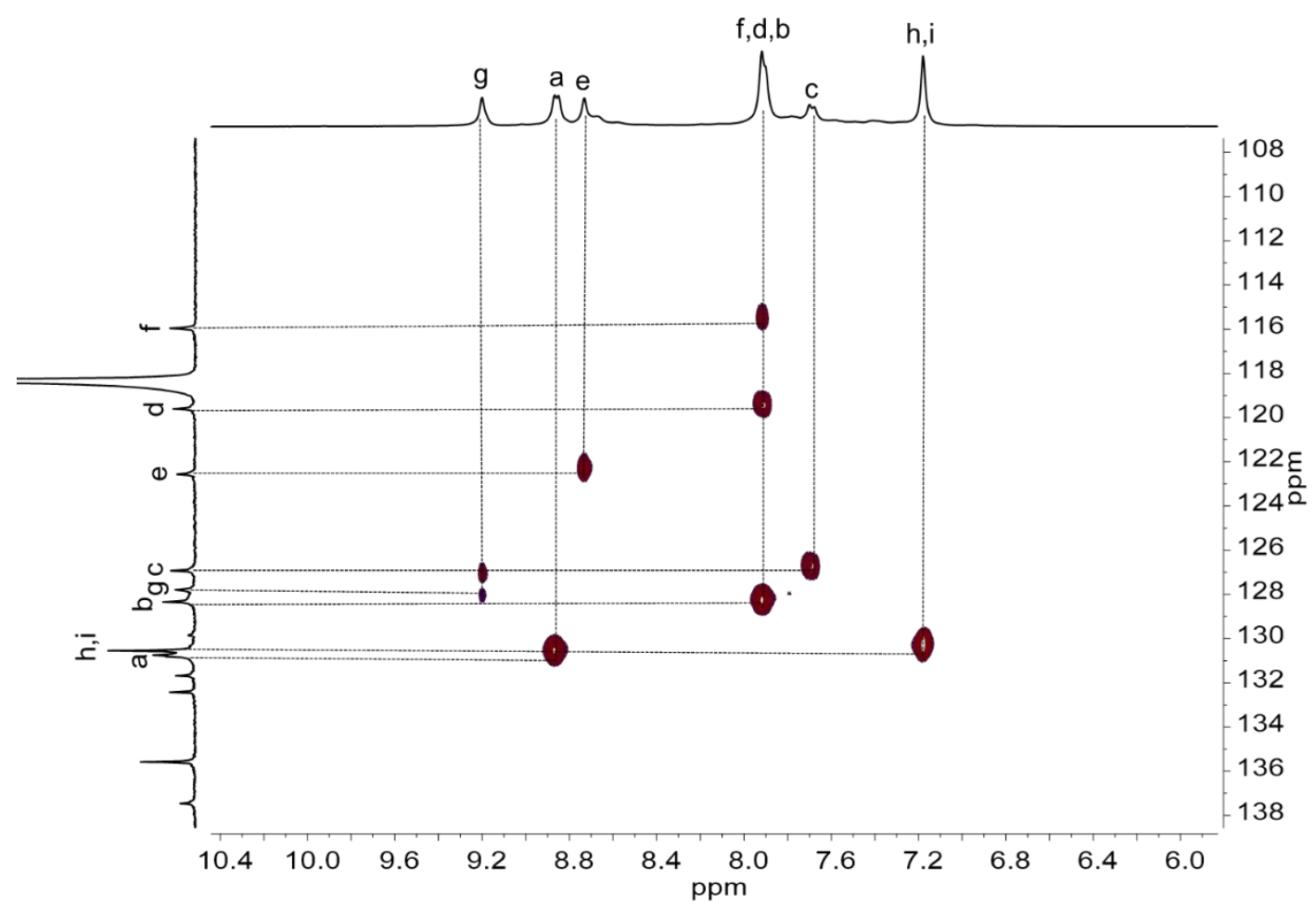

Figure S10. ${ }^{1} \mathrm{H}-{ }^{13} \mathrm{C}$ HSQC spectrum $\left(600 \mathrm{MHz}, \mathrm{CD}_{3} \mathrm{CN}\right)$ of $\mathrm{H}_{3}-\mathbf{1 b}\left(\mathrm{PF}_{6}\right)_{3}$.

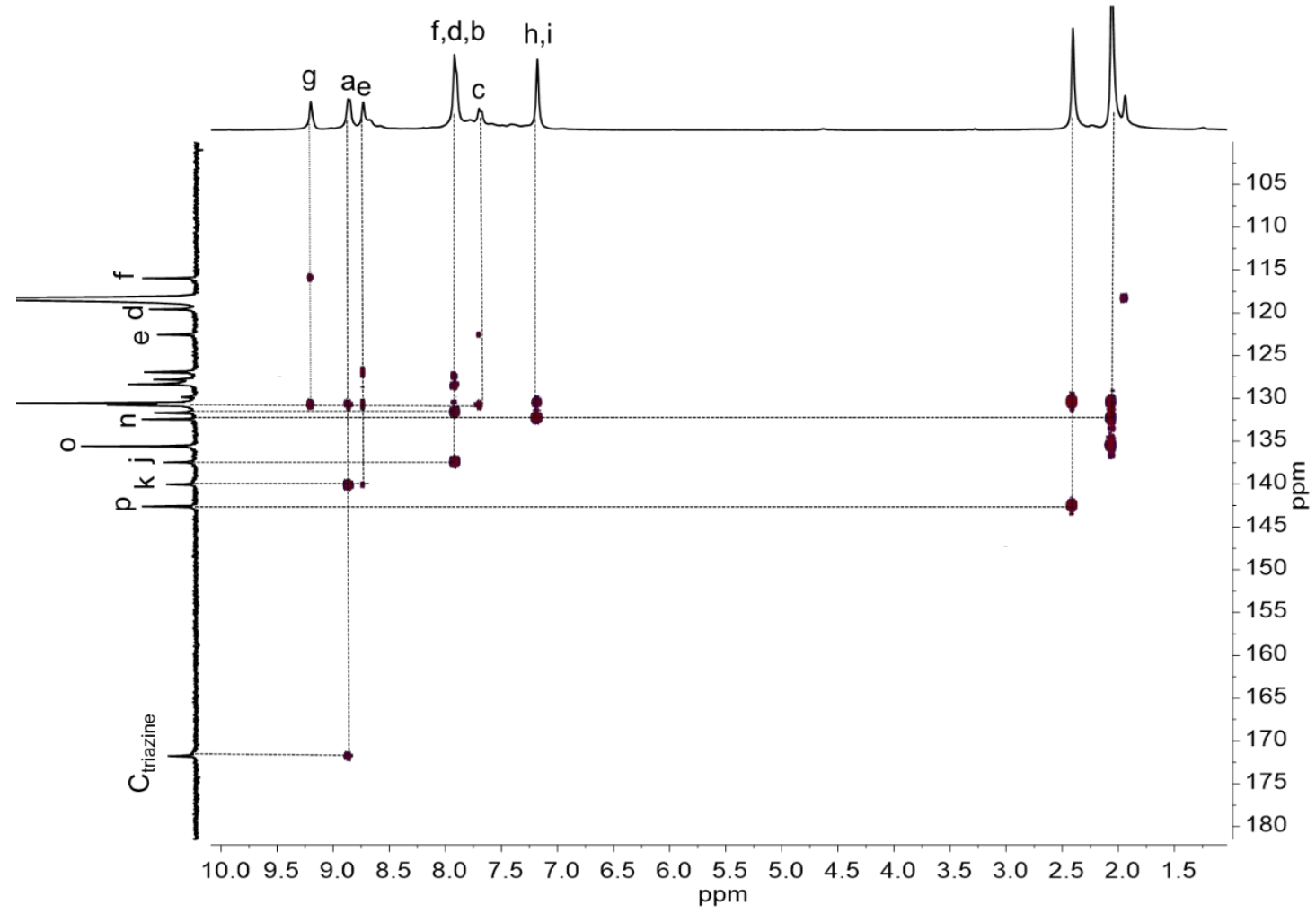

Figure S11. ${ }^{1} \mathrm{H}^{13} \mathrm{C} \mathrm{HMBC}$ spectrum $\left(600 \mathrm{MHz}, \mathrm{CD}_{3} \mathrm{CN}\right)$ of $\mathrm{H}_{3}-\mathbf{1 b}\left(\mathrm{PF}_{6}\right)_{3}$. 


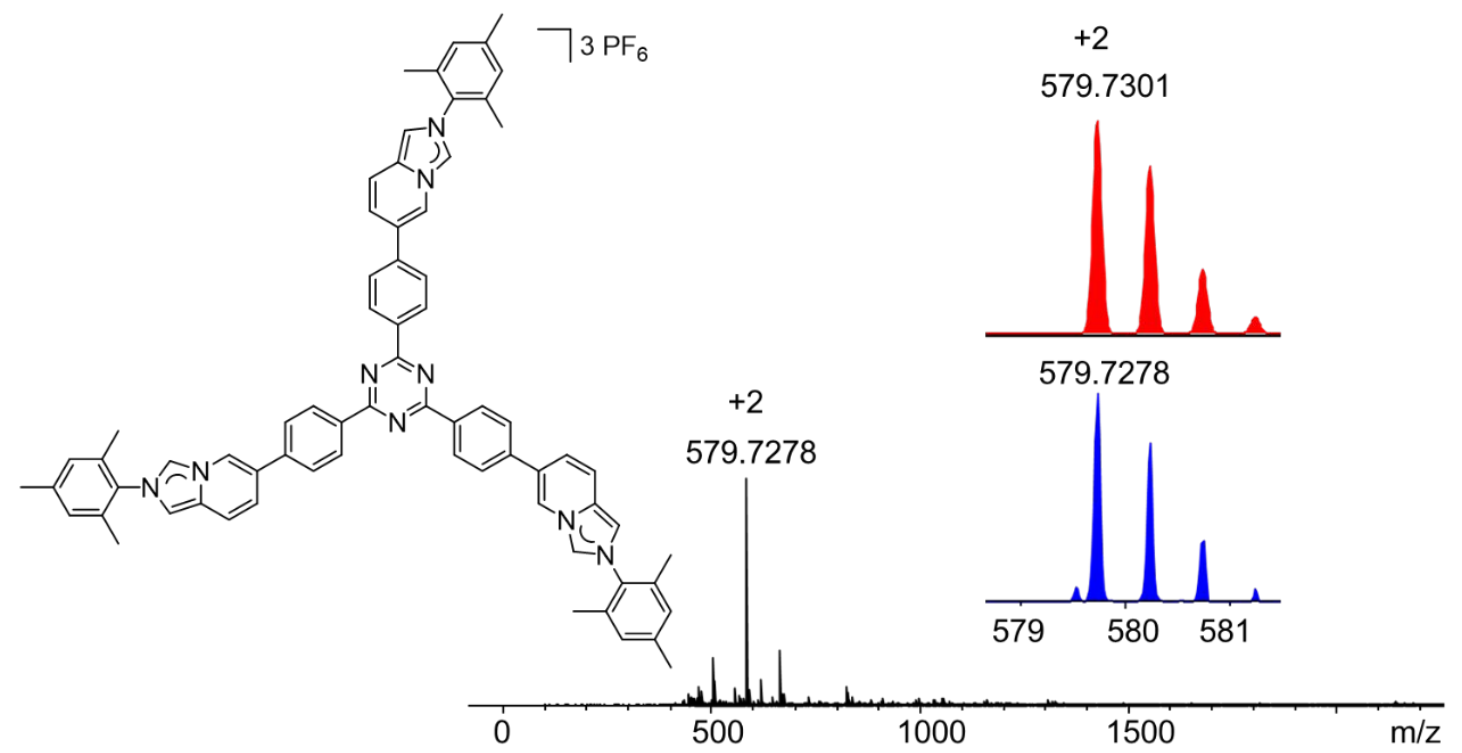

Figure S12. HR-ESI mass spectrum of $\mathrm{H}_{3}-\mathbf{1} \mathbf{b}\left(\mathrm{PF}_{6}\right)_{3}$ with isotope distribution for selected peaks (experimental in blue, calculated in red, $\mathrm{PF}_{6}{ }^{-}$counterions).

\subsection{Synthesis of compound $\mathrm{H}_{3}-1 \mathrm{c}\left(\mathrm{PF}_{6}\right)_{3}$}

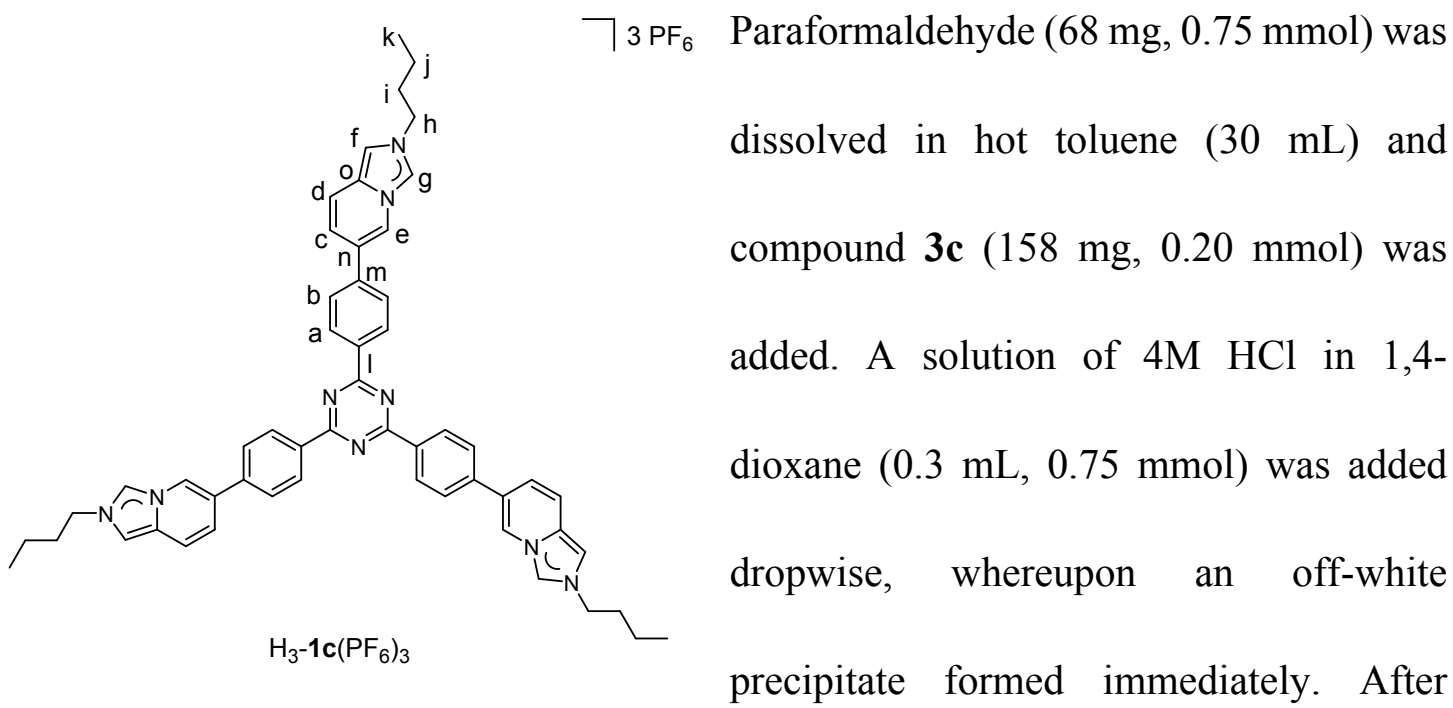

stirring for $24 \mathrm{~h}$ at $43{ }^{\circ} \mathrm{C}$, the precipitate was filtered and washed twice with diethyl ether $(2 \times 2 \mathrm{~mL})$. To remove unreacted paraformaldehyde, the residue was dissolved in methanol and filtered. A solution of $\mathrm{NH}_{4} \mathrm{PF}_{6}$ was then added and the mixture stirred for $8 \mathrm{~h}$ at ambient temperature to precipitate compound $\mathrm{H}_{3}-\mathbf{1} \mathbf{c}\left(\mathrm{PF}_{6}\right)_{3}$ as an off-white solid. 
Yield: $177 \mathrm{mg}(0.14 \mathrm{mmol}, 68 \%) .{ }^{1} \mathrm{H} \mathrm{NMR}\left(600 \mathrm{MHz}, \mathrm{CD}_{3} \mathrm{CN}\right) \delta=9.08\left(\mathrm{~s}, 3 \mathrm{H}, \mathrm{H}_{\mathrm{g}}\right)$, $8.74\left(\mathrm{~d}, J=8.0 \mathrm{~Hz}, 6 \mathrm{H}, \mathrm{H}_{\mathrm{a}}\right), 8.57\left(\mathrm{~s}, 3 \mathrm{H}, \mathrm{H}_{\mathrm{e}}\right), 7.81\left(\mathrm{~s}, 3 \mathrm{H}, \mathrm{H}_{\mathrm{f}}\right), 7.77(\mathrm{~d}, J=8.0 \mathrm{~Hz}, 6 \mathrm{H}$, $\left.\mathrm{H}_{\mathrm{b}}\right), 7.71\left(\mathrm{~d}, J=9.2 \mathrm{~Hz}, 3 \mathrm{H}, \mathrm{H}_{\mathrm{d}}\right), 7.52\left(\mathrm{~d}, J=9.2 \mathrm{~Hz}, 3 \mathrm{H}, \mathrm{H}_{\mathrm{c}}\right), 4.45(\mathrm{t}, J=7.9 \mathrm{~Hz}, 6 \mathrm{H}$, $\left.\mathrm{H}_{\mathrm{h}}\right), 1.99-1.95\left(\mathrm{~m}, 6 \mathrm{H}, \mathrm{H}_{\mathrm{i}}\right), 1.45-1.39\left(\mathrm{~m}, 6 \mathrm{H}, \mathrm{H}_{\mathrm{j}}\right), 1.00\left(\mathrm{t}, J=7.5 \mathrm{~Hz}, 9 \mathrm{H}, \mathrm{H}_{\mathrm{k}}\right)$ ppm. ${ }^{13} \mathrm{C}\left\{{ }^{1} \mathrm{H}\right\}$ NMR $\left(150 \mathrm{MHz}, \mathrm{CD}_{3} \mathrm{CN}\right) \delta=171.7\left(\mathrm{C}_{\text {triazine }}\right), 139.9\left(\mathrm{C}_{\mathrm{m}}\right), 137.2\left(\mathrm{C}_{\mathrm{l}}\right), 130.9$ $\left(C_{n}\right), 130.5\left(C_{a}\right), 130.2\left(C_{o}\right), 128.1\left(C_{b}\right), 126.7\left(C_{g}\right), 126.1\left(C_{c}\right), 121.9\left(C_{e}\right), 119.1\left(C_{d}\right)$, $114.3\left(\mathrm{C}_{\mathrm{f}}\right), 51.6\left(\mathrm{C}_{\mathrm{h}}\right), 33.0\left(\mathrm{C}_{\mathrm{i}}\right), 20.0\left(\mathrm{C}_{\mathrm{j}}\right), 13.6\left(\mathrm{C}_{\mathrm{k}}\right)$ ppm. HRMS (ESI, positive ions): $m / z=486.7084\left(\right.$ calcd for $\left.\left[\mathrm{H}_{3}-\mathbf{1} \mathbf{c}\left(\mathrm{PF}_{6}\right)\right]^{2+} 486.7067\right), 276.1626\left(\right.$ calcd for $\left[\mathrm{H}_{3}-\mathbf{1 c}\right]^{3+}$ 276.1495).

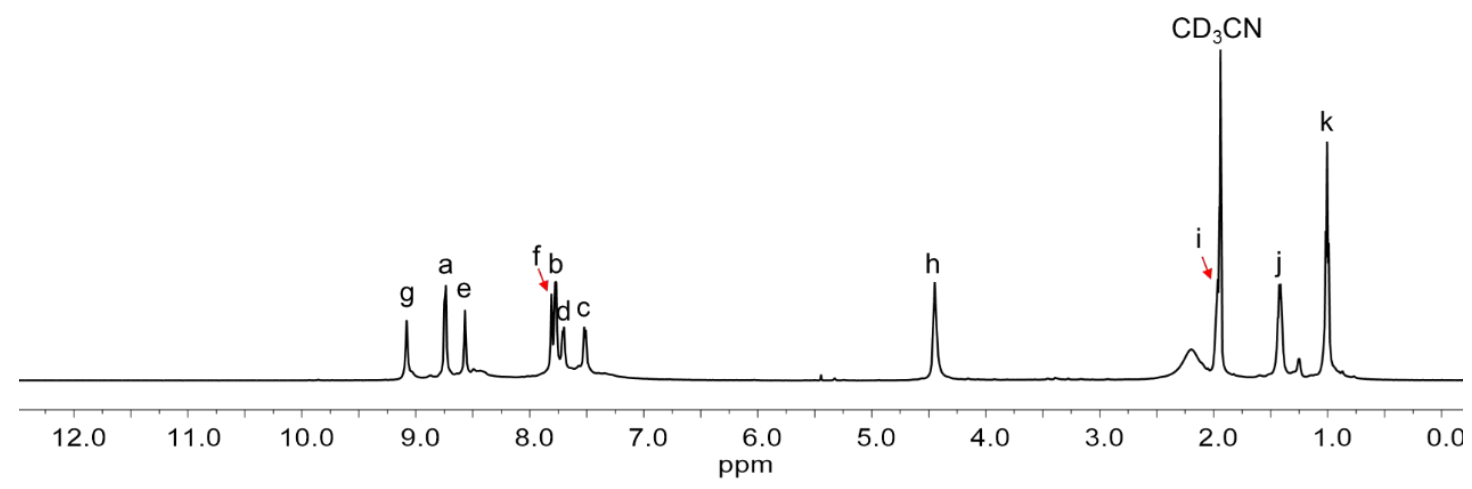

Figure S13. ${ }^{1} \mathrm{H}$ NMR spectrum $\left(600 \mathrm{MHz}, \mathrm{CD}_{3} \mathrm{CN}\right)$ of $\mathrm{H}_{3}-\mathbf{1 c}\left(\mathrm{PF}_{6}\right)_{3}$.

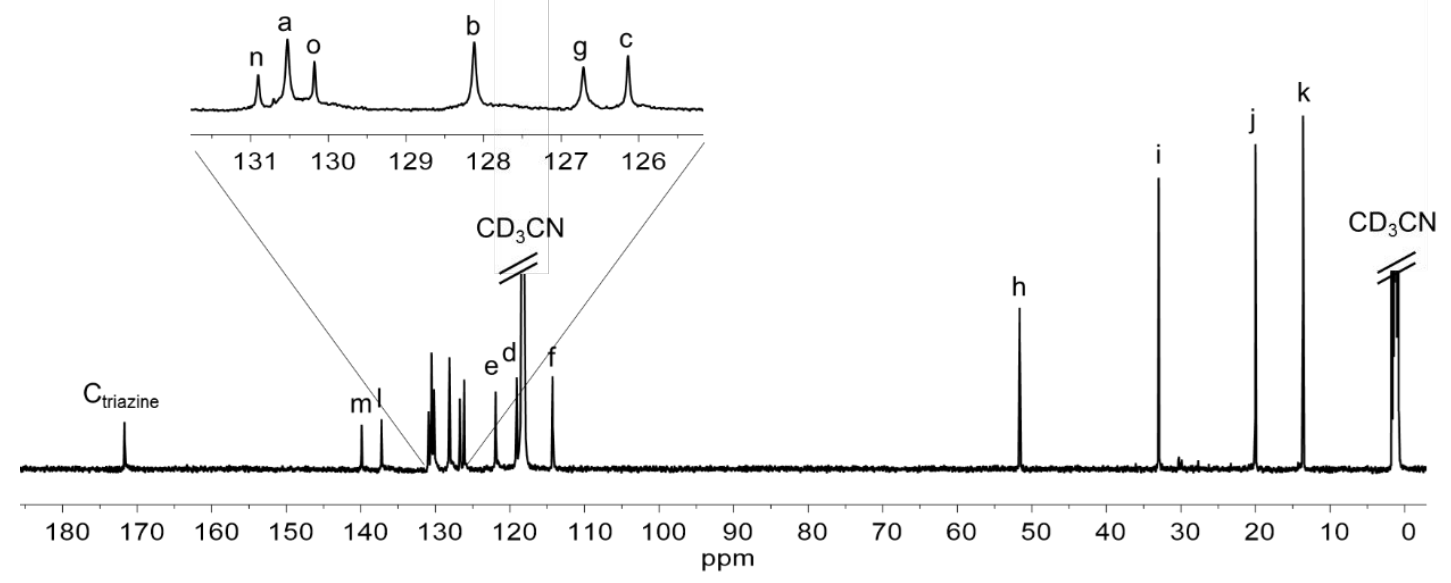

Figure S14. ${ }^{13} \mathrm{C}\left\{{ }^{1} \mathrm{H}\right\}$ NMR spectrum $\left(150 \mathrm{MHz}, \mathrm{CD}_{3} \mathrm{CN}\right)$ of $\mathrm{H}_{3}-1 \mathrm{c}\left(\mathrm{PF}_{6}\right)_{3}$. 


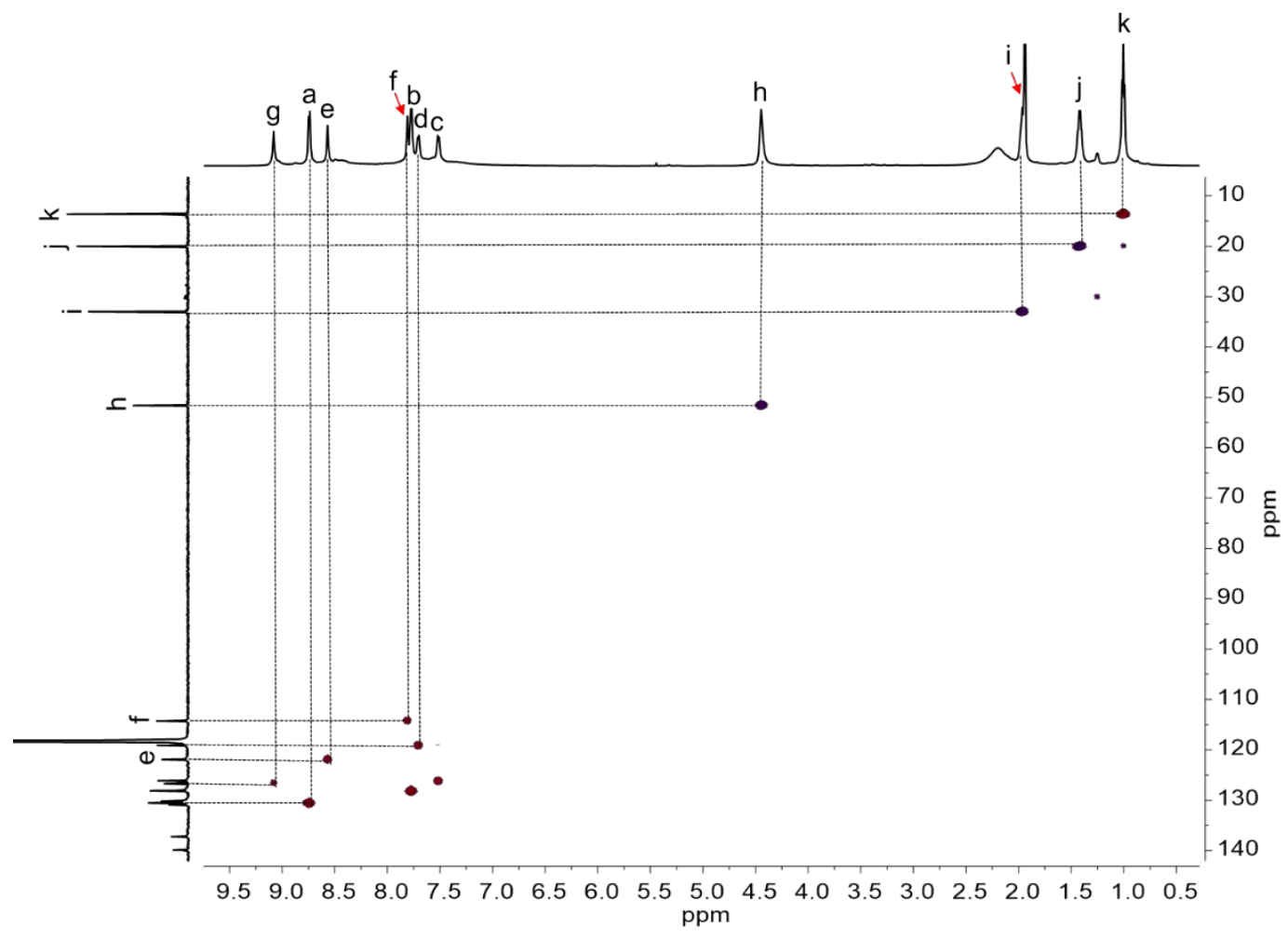

Figure S15. ${ }^{1} \mathrm{H}_{-}{ }^{13} \mathrm{C}$ HSQC spectrum $\left(600 \mathrm{MHz}, \mathrm{CD}_{3} \mathrm{CN}\right)$ of compound $\mathrm{H}_{3}-\mathbf{1} \mathbf{c}\left(\mathrm{PF}_{6}\right)_{3}$.

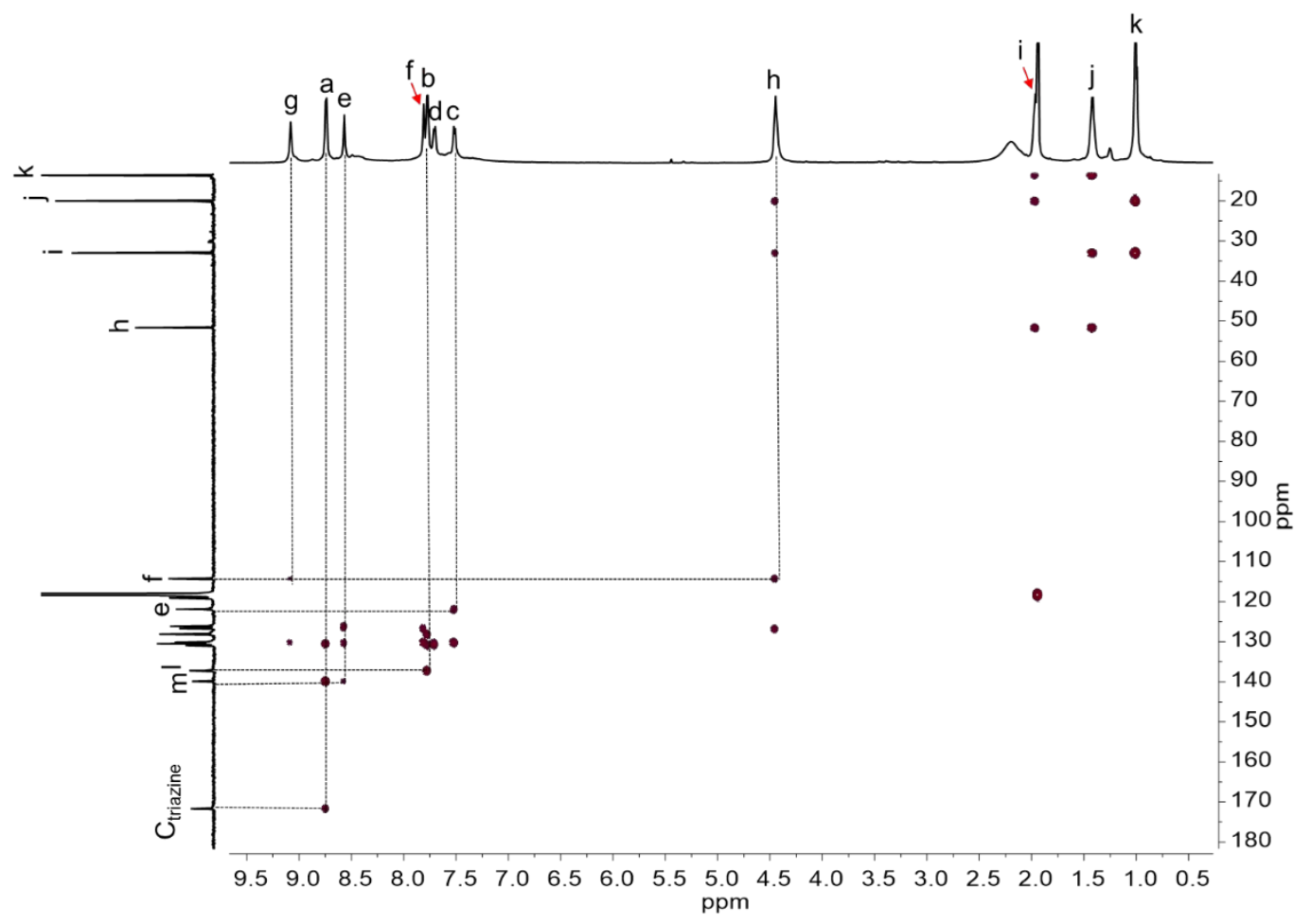

Figure S16. ${ }^{1} \mathrm{H}-{ }^{13} \mathrm{C} \mathrm{HMBC}$ spectrum $\left(600 \mathrm{MHz}, \mathrm{CD}_{3} \mathrm{CN}\right)$ of $\mathrm{H}_{3}-\mathbf{1 c}\left(\mathrm{PF}_{6}\right)_{3}$. 


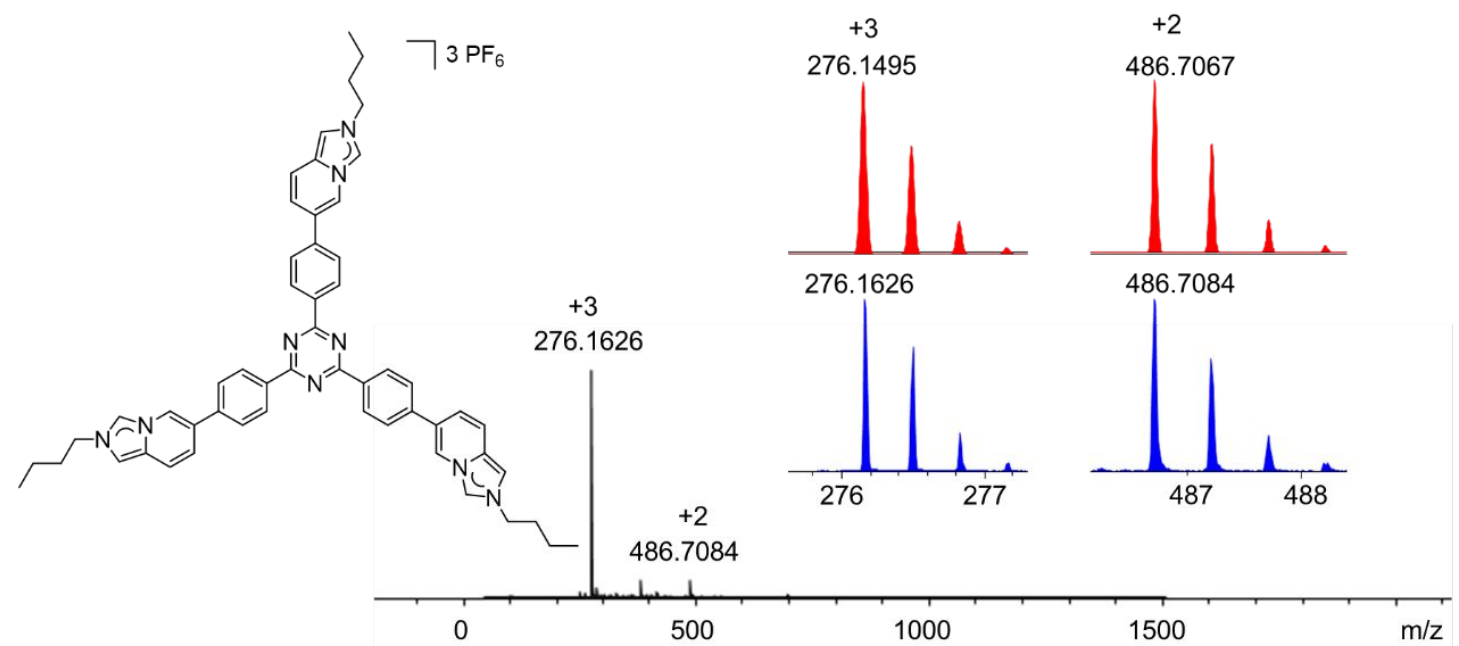

Figure S17. HR-ESI mass spectrum of $\mathrm{H}_{3}-\mathbf{1} \mathbf{c}\left(\mathrm{PF}_{6}\right)_{3}$ with isotope distribution for selected peaks (experimental in blue, calculated in red, $\mathrm{PF}_{6}^{-}$counterions).

\section{Synthesis of complexes $\left[\mathrm{Ag}_{3}(\mathrm{~L})_{2}\right]_{2}\left(\mathrm{PF}_{6}\right)_{6}(\mathrm{~L}=1 \mathrm{a}-1 \mathrm{c})$}

\section{General synthetic procedure of complexes $\left[\mathrm{Ag}_{3}(\mathrm{~L})_{2}\right]_{2}\left(\mathrm{PF}_{6}\right)_{6}(\mathrm{~L}=1 \mathrm{a}-1 \mathrm{c})$}

To a solution of one of the imidazo[1,5- $a$ ]pyridinium salts (in $20 \mathrm{~mL}$ of $\mathrm{CH}_{3} \mathrm{CN}$ ) was added excess $\mathrm{Ag}_{2} \mathrm{O}$ under a nitrogen atmosphere. The resulting suspension was heated to $55{ }^{\circ} \mathrm{C}$ for $20 \mathrm{~h}$ with exclusion of light. Upon cooling to ambient temperature a suspension was obtained. This suspension was filtered slowly through a pad of Celite to give a clear solution. The filtrate was concentrated to $2 \mathrm{~mL}$, and diethyl ether (20 $\mathrm{mL}$ ) was added. A gray precipitate formed, which was isolated by filtration, washed with diethyl ether and dried in vacuo to afford the complexes of type $\left[\mathrm{Ag}_{3}(\mathbf{L})_{2}\right]_{2}\left(\mathrm{PF}_{6}\right)_{6}$ $(\mathbf{L}=\mathbf{1 a}-\mathbf{1 c})$ 


\subsection{Synthesis of complex $\left[\operatorname{Ag}_{3}(1 \mathrm{a})_{2}\right]_{2}\left(\mathrm{PF}_{6}\right)_{6}$}

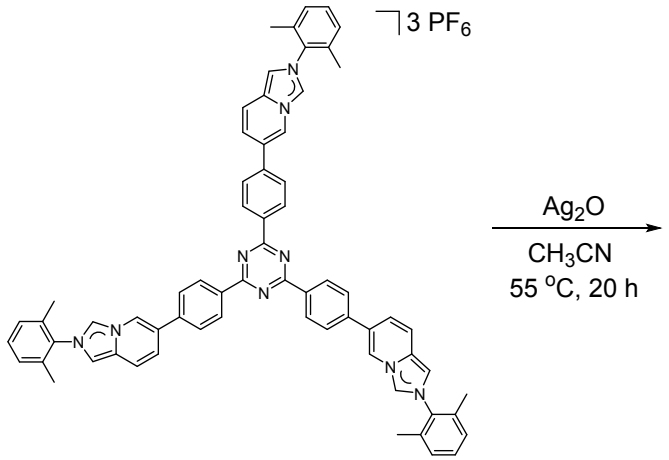

$\mathrm{H}_{3}-1 \mathbf{a}\left(\mathrm{PF}_{6}\right)_{3}$

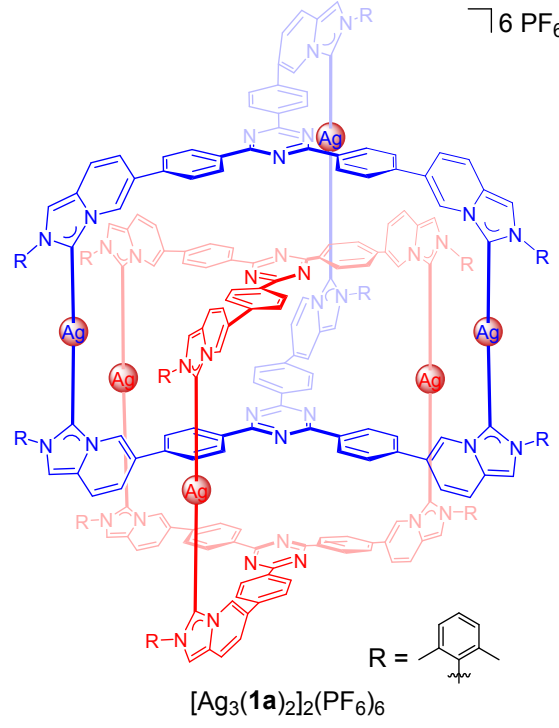

Scheme S2. Synthesis of Complex $\left[\mathrm{Ag}_{3}(\mathbf{1 a})_{2}\right]_{2}\left(\mathrm{PF}_{6}\right)_{6}$.

Complex $\left[\mathrm{Ag}_{3}(\mathbf{1 a})_{2}\right]_{2}\left(\mathrm{PF}_{6}\right)_{6}$ was prepared from $\mathrm{H}_{3}-\mathbf{1 a}\left(\mathrm{PF}_{6}\right)_{3}(30 \mathrm{mg}, 0.021 \mathrm{mmol})$ and $\mathrm{Ag}_{2} \mathrm{O}$ (22 mg, $\left.0.096 \mathrm{mmol}\right)$. Yield: $26 \mathrm{mg}(0.0048 \mathrm{mmol}, 92 \%) .{ }^{1} \mathrm{H}$ NMR (600 MHz, $\left.\mathrm{CD}_{3} \mathrm{CN}\right) \delta=8.94\left(\mathrm{~s}, 6 \mathrm{H}, \mathrm{H}_{\mathrm{e}}\right), 8.69\left(\mathrm{~s}, 6 \mathrm{H}, \mathrm{H}_{\mathrm{e}^{\prime}}\right), 7.96\left(\mathrm{~d}, J=7.7 \mathrm{~Hz}, 12 \mathrm{H}, \mathrm{H}_{\mathrm{a}}\right), 7.80-7.75$ $\left(\mathrm{m}, 18 \mathrm{H}, \mathrm{H}_{\mathrm{b}, \mathrm{d}}\right), 7.64\left(\mathrm{~s}, 6 \mathrm{H}, \mathrm{H}_{\mathrm{f}}\right), 7.56\left(\mathrm{~s}, 6 \mathrm{H}, \mathrm{H}_{\mathrm{f}^{\prime}}\right), 7.55-7.50\left(\mathrm{~m}, 24 \mathrm{H}, \mathrm{H}_{\mathrm{a}^{\prime}, \mathrm{b}^{\prime}}\right), 7.42(\mathrm{~d}, J$ $\left.=9.2 \mathrm{~Hz}, 6 \mathrm{H}, \mathrm{H}_{\mathrm{c}}\right), 7.39\left(\mathrm{~d}, J=7.7 \mathrm{~Hz}, 12 \mathrm{H}, \mathrm{H}_{\mathrm{h}}\right), 7.31-7.20\left(\mathrm{~m}, 30 \mathrm{H}, \mathrm{H}_{\mathrm{h}^{\prime}, \mathrm{c}^{\prime}, \mathrm{i}, \mathrm{i}^{\prime}}\right), 6.92(\mathrm{~d}$, $\left.J=9.2 \mathrm{~Hz}, 6 \mathrm{H}, \mathrm{H}_{\mathrm{d}^{\prime}}\right), 2.40\left(\mathrm{~s}, 18 \mathrm{H}, \mathrm{CH}_{3}\right), 1.88\left(\mathrm{~s}, 18 \mathrm{H}, \mathrm{CH}_{3}\right), 1.68\left(\mathrm{~s}, 18 \mathrm{H}, \mathrm{CH}_{3}\right), 1.59$ (s, $\left.18 \mathrm{H}, \mathrm{CH}_{3}\right) \mathrm{ppm} .{ }^{13} \mathrm{C}\left\{{ }^{1} \mathrm{H}\right\} \mathrm{NMR}\left(150 \mathrm{MHz}, \mathrm{CD}_{3} \mathrm{CN}\right) \delta=175.7$ (from $\mathrm{HMBC}, \mathrm{C}_{\mathrm{NHC}}$ ), 174.5 (from $\left.\mathrm{HMBC}, \mathrm{C}_{\mathrm{NHC}}\right), 170.6\left(\mathrm{C}_{\text {triazine }}\right), 169.5$ ( $\left.\mathrm{C}_{\text {triazine }}\right), 141.7,140.6,139.2,135.8$, 135.6, 135.5, 135.4, 135.2, 131.6, 131.2, 131.0, 130.9, 130.8, 130.1, $129.3\left(\mathrm{C}_{\mathrm{h}}\right), 129.1$ $\left(\mathrm{C}_{\mathrm{h}}\right), 128.8,128.6,127.4\left(\mathrm{C}_{\mathrm{e}}\right), 127.2\left(\mathrm{C}_{\mathrm{b}}\right), 126.9\left(\mathrm{C}_{\mathrm{e}^{\prime}}\right), 126.1,125.2\left(\mathrm{C}_{\mathrm{c}}\right), 124.1\left(\mathrm{C}_{\mathrm{c}^{\prime}}\right)$, 119.6 $\left(\mathrm{C}_{\mathrm{d}}\right), 118.9\left(\mathrm{C}_{\mathrm{d}^{\prime}}\right), 114.9\left(\mathrm{C}_{\mathrm{f}}\right), 114.7\left(\mathrm{C}_{\mathrm{f}}\right), 18.5\left(\mathrm{CH}_{3}\right), 17.7\left(\mathrm{CH}_{3}\right), 17.4\left(\mathrm{CH}_{3}\right), 17.3$ $\left(\mathrm{CH}_{3}\right)$ ppm. ESI-TOF MS: $m / z=1654.3269$ (calcd for $\left\{\left[\mathrm{Ag}_{3}(\mathbf{1 a})_{2}\right]_{2}\left(\mathrm{PF}_{6}\right)_{3}\right\}^{3+}$ 1654.3473), 1204.2615 (calcd for $\left.\left\{\left[\mathrm{Ag}_{3}(\mathbf{1 a})_{2}\right]_{2}\left(\mathrm{PF}_{6}\right)_{2}\right\}^{4+} 1204.2675\right), 934.4138$ (calcd 
for $\left\{\left[\mathrm{Ag}_{3}(\mathbf{1 a})_{2}\right]_{2}\left(\mathrm{PF}_{6}\right)\right\}^{5+}$ 934.4212).
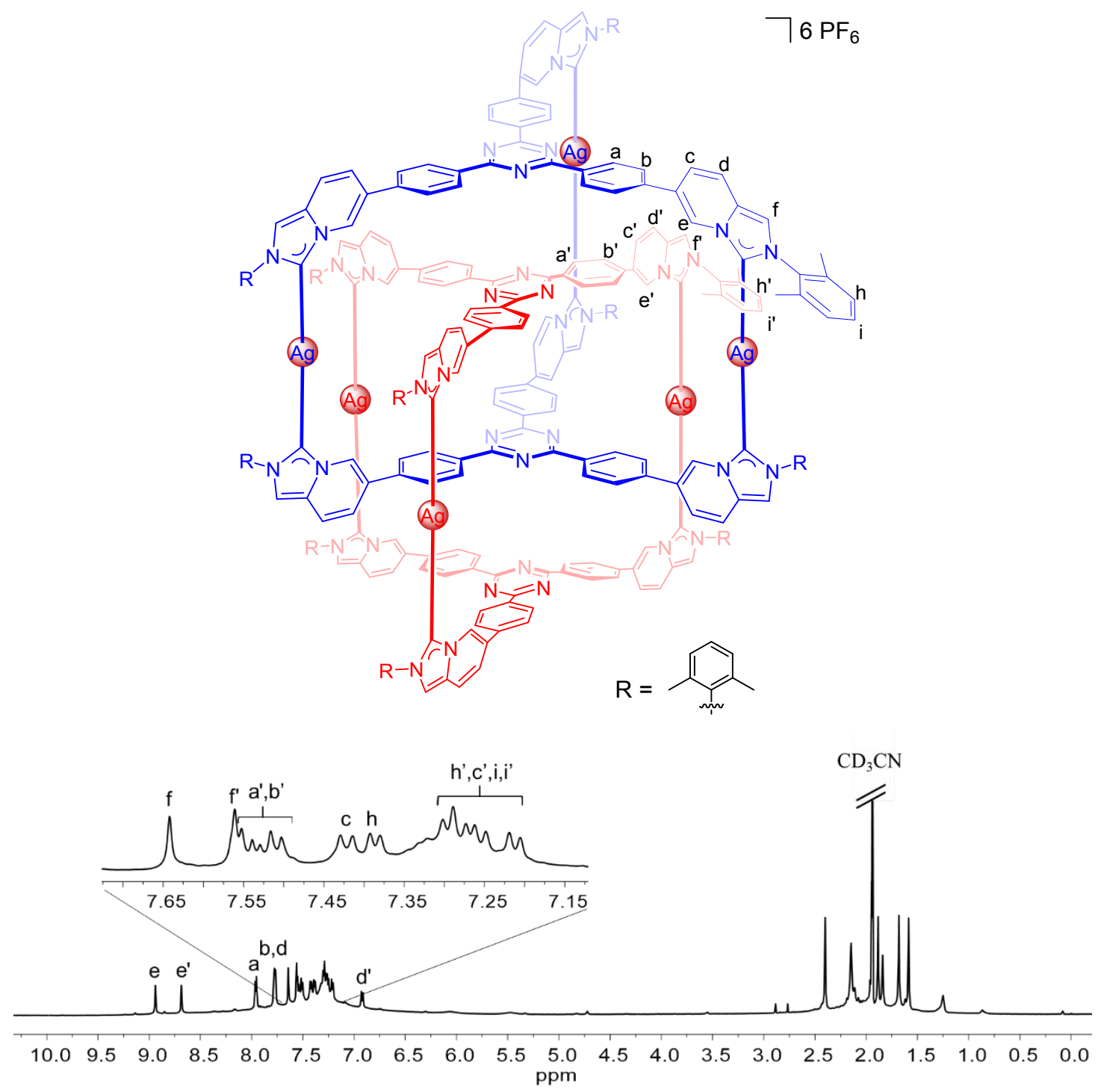

Figure S18. ${ }^{1} \mathrm{H} \mathrm{NMR}$ spectrum $\left(600 \mathrm{MHz}, \mathrm{CD}_{3} \mathrm{CN}\right)$ of $\left[\mathrm{Ag}_{3}(\mathbf{1 a})_{2}\right]_{2}\left(\mathrm{PF}_{6}\right)_{6}$.

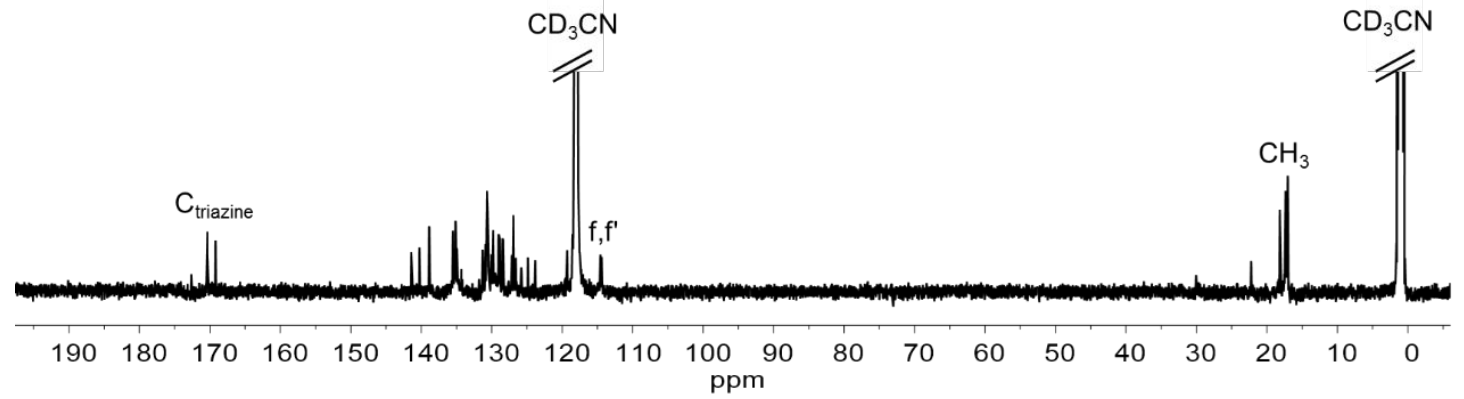

Figure S19. ${ }^{13} \mathrm{C}\left\{{ }^{1} \mathrm{H}\right\}$ NMR spectrum $\left(150 \mathrm{MHz}, \mathrm{CD}_{3} \mathrm{CN}\right)$ of $\left[\mathrm{Ag}_{3}(\mathbf{1 a})_{2}\right]_{2}\left(\mathrm{PF}_{6}\right)_{6}$. 


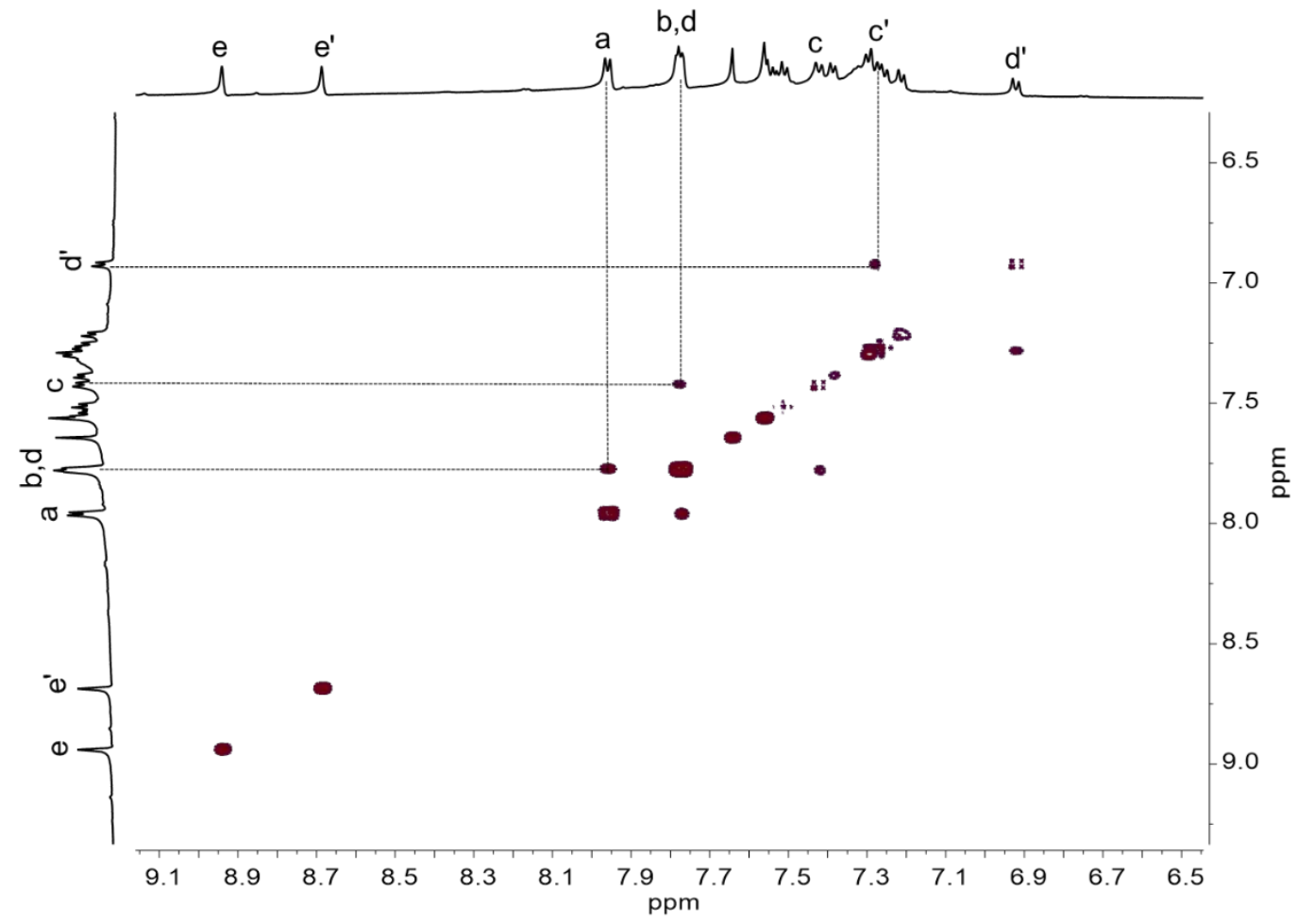

Figure S20. ${ }^{1} \mathrm{H}-{ }^{1} \mathrm{H}$ COSY spectrum $\left(600 \mathrm{MHz}, \mathrm{CD}_{3} \mathrm{CN}\right)$ of $\left[\mathrm{Ag}_{3}(\mathbf{1 a})_{2}\right]_{2}\left(\mathrm{PF}_{6}\right)_{6}$.

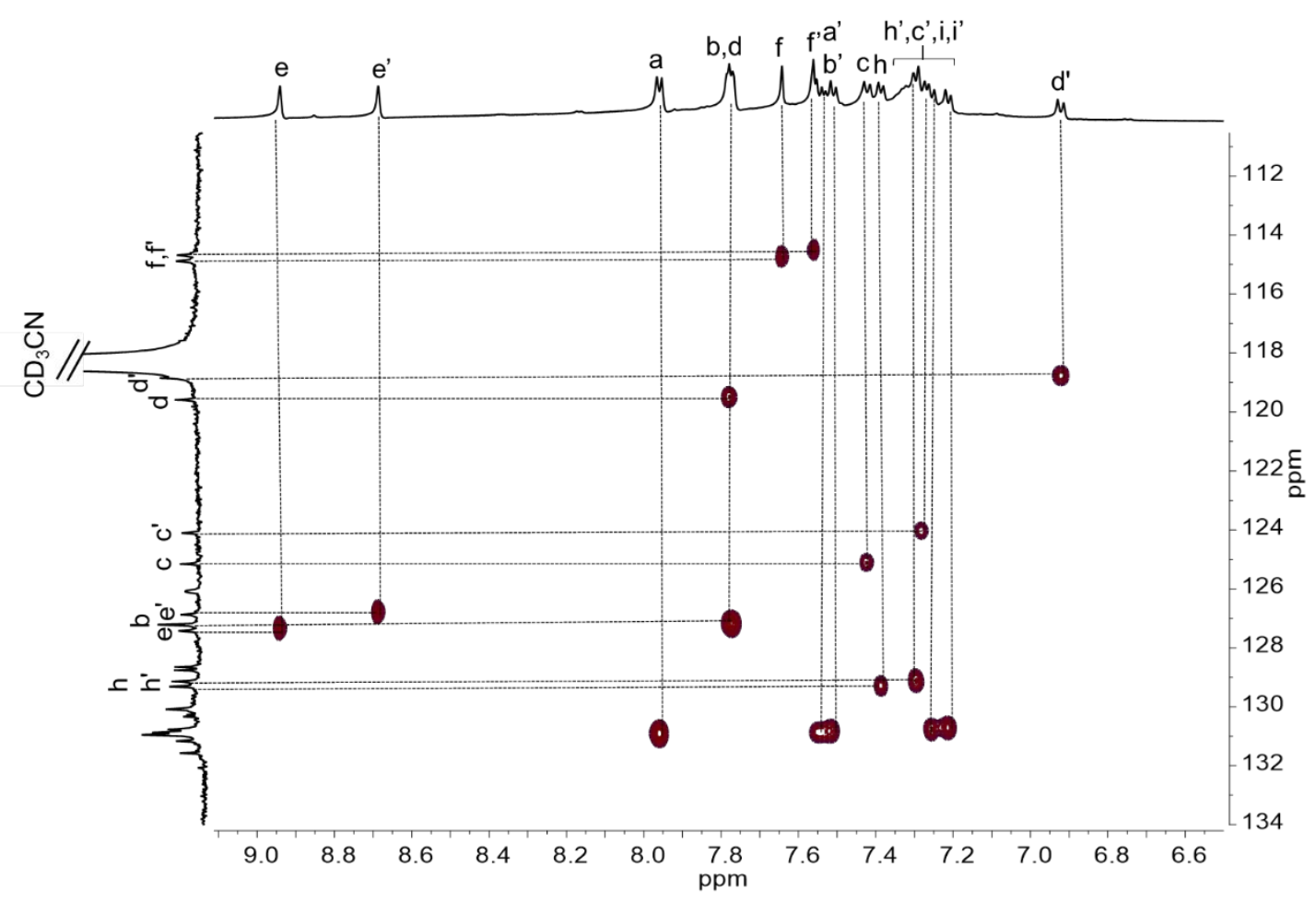

Figure S21. ${ }^{1} \mathrm{H}-{ }^{13} \mathrm{C}$ HSQC spectrum $\left(600 \mathrm{MHz}, \mathrm{CD}_{3} \mathrm{CN}\right)$ of $\left[\mathrm{Ag}_{3}(\mathbf{1 a})_{2}\right]_{2}\left(\mathrm{PF}_{6}\right)_{6}$. 


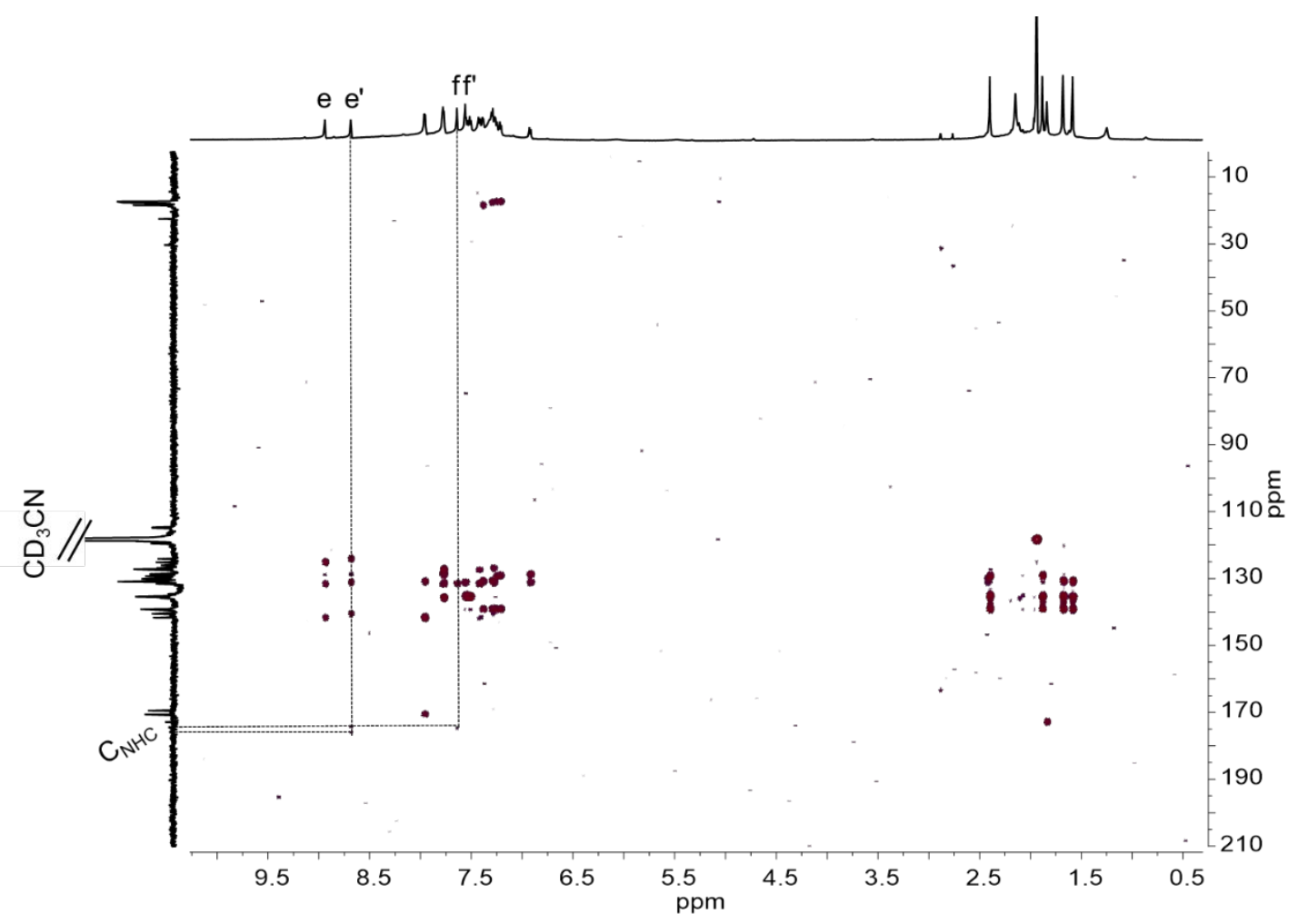

Figure S22. ${ }^{1} \mathrm{H}-{ }^{13} \mathrm{C}$ HMBC spectrum $\left(600 \mathrm{MHz}, \mathrm{CD}_{3} \mathrm{CN}\right)$ of $\left[\mathrm{Ag}_{3}(\mathbf{1 a})_{2}\right]_{2}\left(\mathrm{PF}_{6}\right)_{6}$.

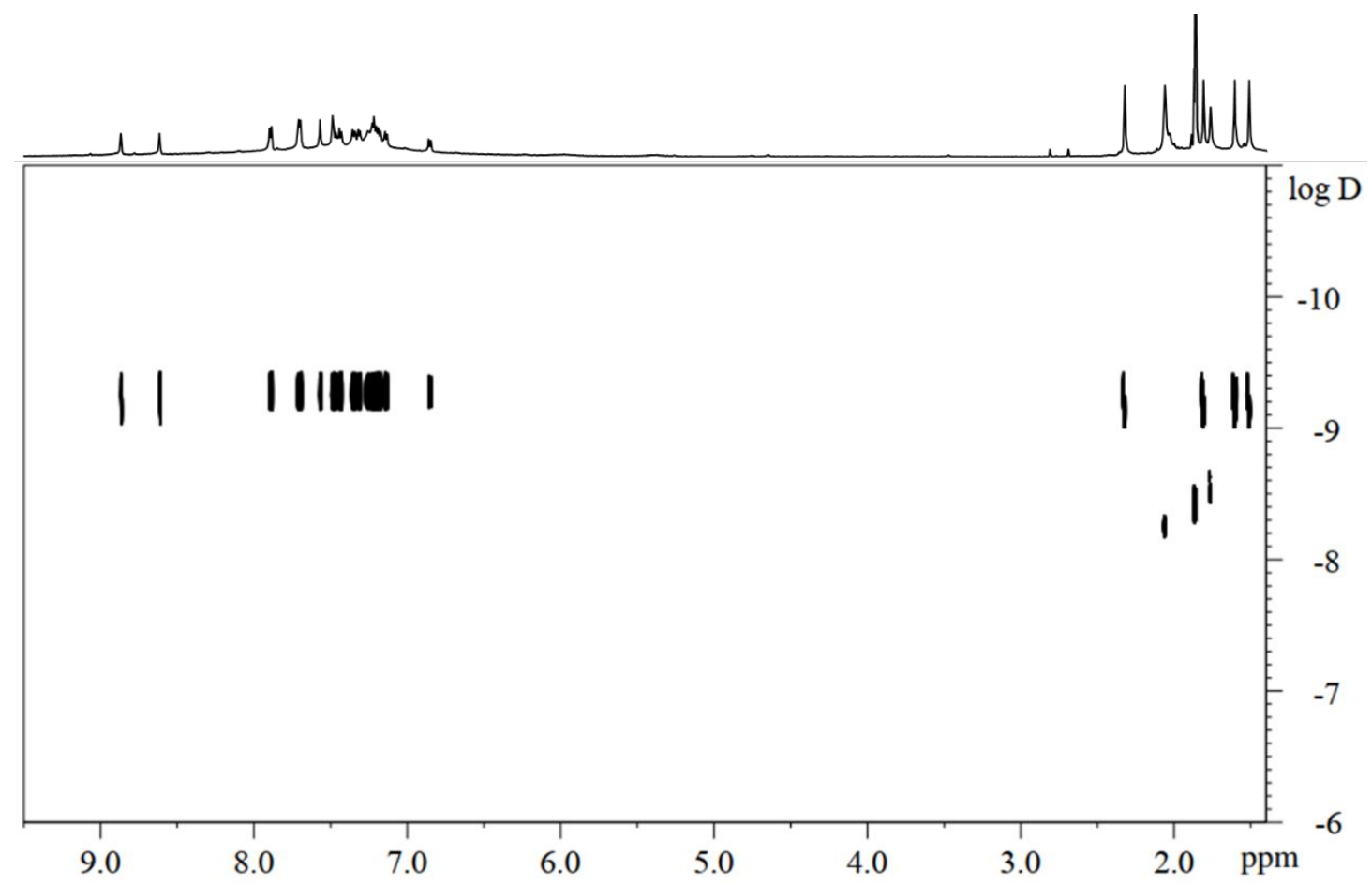

Figure S23. ${ }^{1} \mathrm{H}$ DOSY spectrum $\left(600 \mathrm{MHz}, \mathrm{CD}_{3} \mathrm{CN}\right)$ of $\left[\mathrm{Ag}_{3}(\mathbf{1 a})_{2}\right]_{2}\left(\mathrm{PF}_{6}\right)_{6}$. 


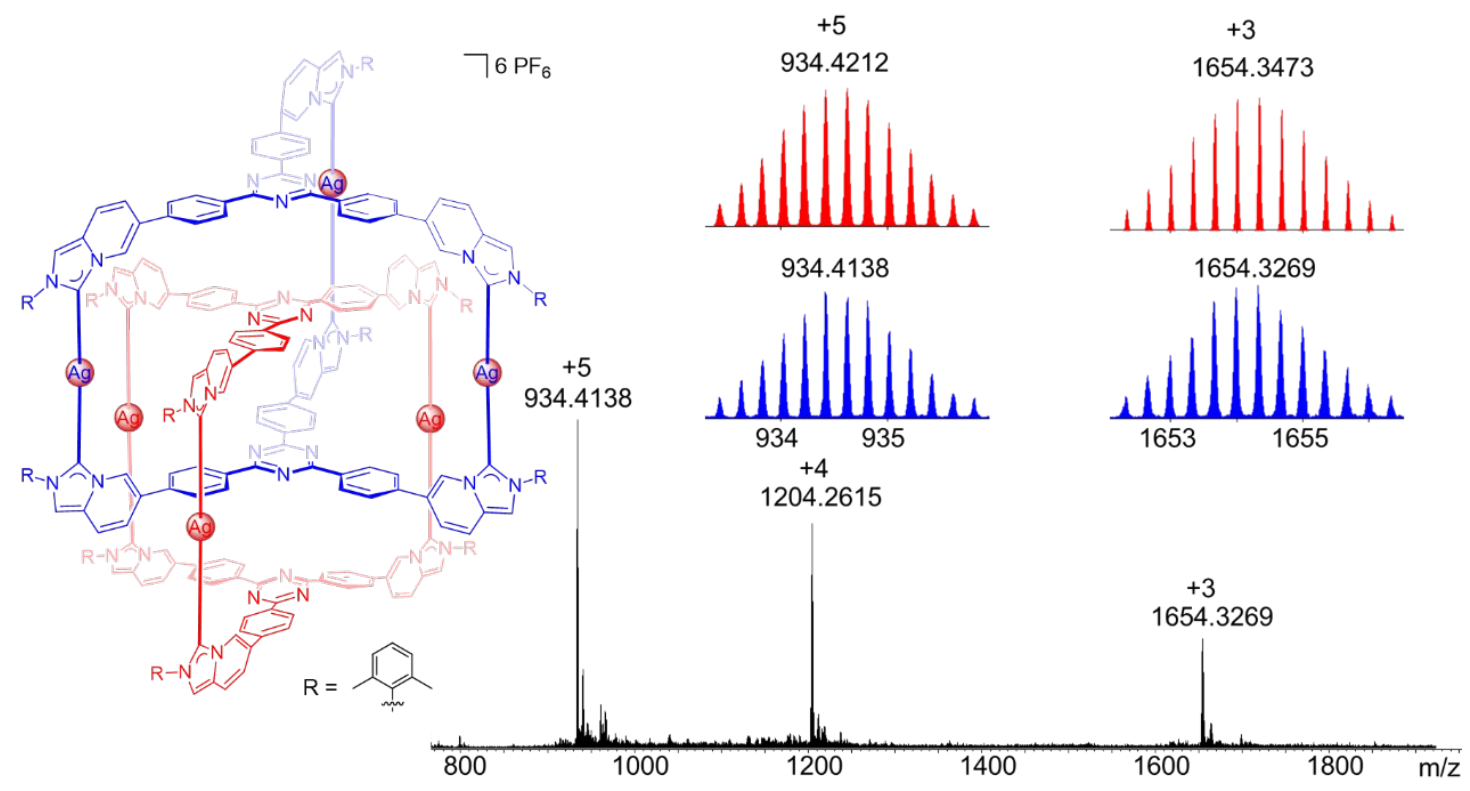

Figure S24. ESI-TOF mass spectrum of $\left[\operatorname{Ag}_{3}\left(\mathbf{1 a}_{2}\right]_{2}\left(\mathrm{PF}_{6}\right)_{6}\right.$ with isotope distribution for selected peaks (experimental in blue, calculated in red, $\mathrm{PF}_{6}{ }^{-}$counterions).

\subsection{Synthesis of complex $\left[\mathrm{Ag}_{3}(1 \mathrm{~b})_{2}\right]_{2}\left(\mathrm{PF}_{6}\right)_{6}$}

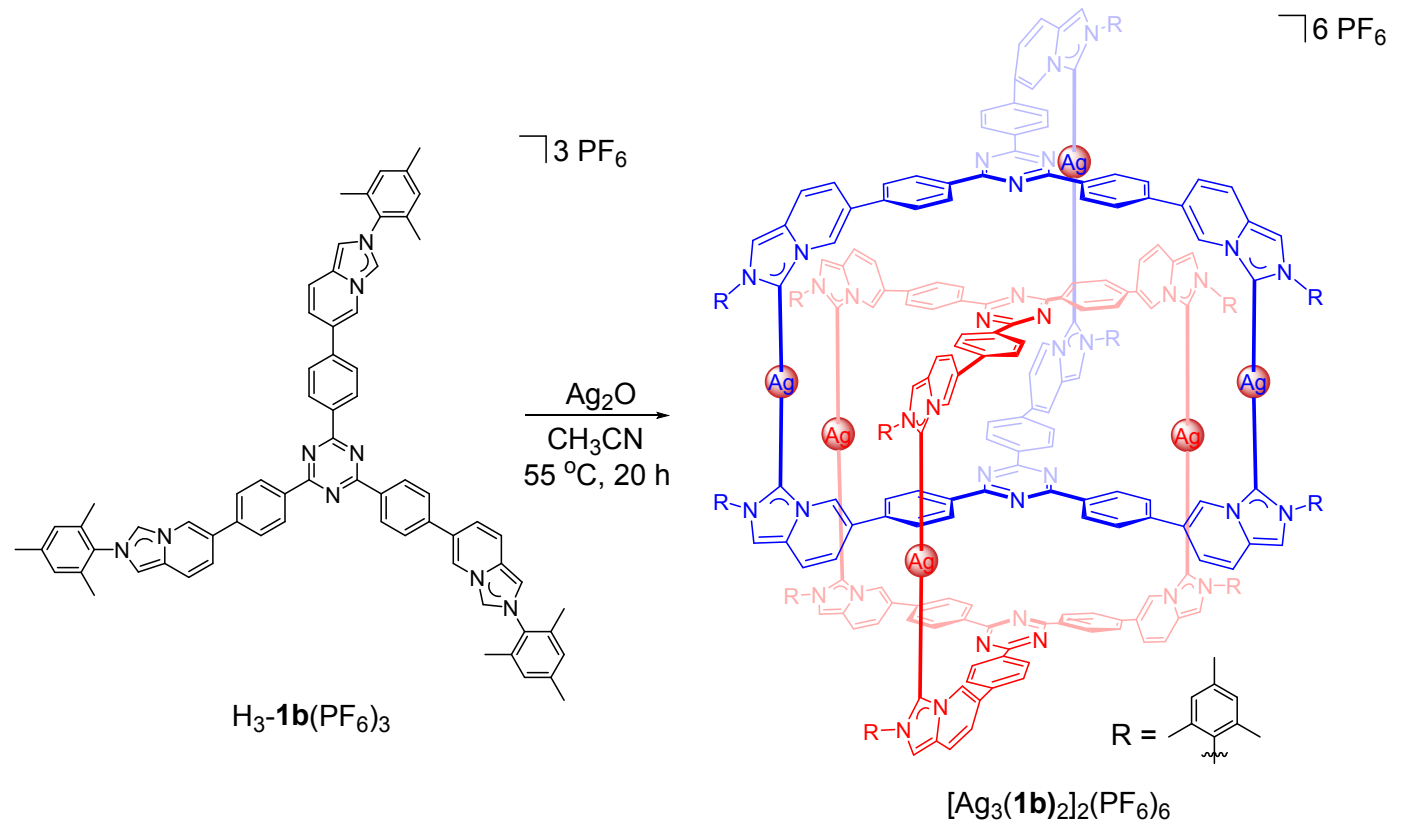

Scheme S3. Synthesis of Complex $\left[\mathrm{Ag}_{3}(\mathbf{1 b})_{2}\right]_{2}\left(\mathrm{PF}_{6}\right)_{6}$.

Complex $\left[\mathrm{Ag}_{3}(\mathbf{1 b})_{2}\right]_{2}\left(\mathrm{PF}_{6}\right)_{6}$ was prepared from $\mathrm{H}_{3}-\mathbf{1 b}\left(\mathrm{PF}_{6}\right)_{3}(30 \mathrm{mg}, 0.021 \mathrm{mmol})$ and $\mathrm{Ag}_{2} \mathrm{O}$ (22 mg, $\left.0.095 \mathrm{mmol}\right)$. Yield: $26 \mathrm{mg}(0.0047 \mathrm{mmol}, 90 \%) .{ }^{1} \mathrm{H} \mathrm{NMR}(600 \mathrm{MHz}$, 
$\left.\mathrm{CD}_{3} \mathrm{CN}\right) \delta=8.92\left(\mathrm{~s}, 6 \mathrm{H}, \mathrm{H}_{\mathrm{e}}\right), 8.66\left(\mathrm{~s}, 6 \mathrm{H}, \mathrm{H}_{\mathrm{e}}\right), 7.94\left(\mathrm{~d}, J=7.7 \mathrm{~Hz}, 12 \mathrm{H}, \mathrm{H}_{\mathrm{a}}\right), 7.78-7.75$ $\left(\mathrm{m}, 18 \mathrm{H}, \mathrm{H}_{\mathrm{d}, \mathrm{b}}\right), 7.62\left(\mathrm{~s}, 6 \mathrm{H}, \mathrm{H}_{\mathrm{f}}\right), 7.54\left(\mathrm{~s}, 6 \mathrm{H}, \mathrm{H}_{\mathrm{f}^{\prime}}\right), 7.51-7.48\left(\mathrm{~m}, 12 \mathrm{H}, \mathrm{H}_{\mathrm{a}^{\prime}}\right), 7.41(\mathrm{~d}, J=$ $\left.9.2 \mathrm{~Hz}, 6 \mathrm{H}, \mathrm{H}_{\mathrm{c}}\right), 7.31-7.29\left(\mathrm{~m}, 12 \mathrm{H}, \mathrm{H}_{\mathrm{b}}\right), 7.25\left(\mathrm{~d}, J=9.2 \mathrm{~Hz}, 6 \mathrm{H}, \mathrm{H}_{\mathrm{c}^{\prime}}\right), 7.21(\mathrm{~s}, 6 \mathrm{H}$, $\left.\mathrm{H}_{\mathrm{h} / \mathrm{h}^{\prime} / \mathrm{i} / \mathrm{i}^{\prime}}\right), 7.12\left(\mathrm{~s}, 6 \mathrm{H}, \mathrm{H}_{\mathrm{h} / \mathrm{h}^{\prime} / \mathrm{i} / \mathrm{i}^{\prime}}\right), 7.04\left(\mathrm{~s}, 6 \mathrm{H}, \mathrm{H}_{\mathrm{h} / \mathrm{h}^{\prime} / \mathrm{i} / \mathrm{i}^{\prime}}\right), 6.99\left(\mathrm{~s}, 6 \mathrm{H}, \mathrm{H}_{\mathrm{h} / \mathrm{h}^{\prime} / \mathrm{i} / \mathrm{i}^{\prime}}\right), 6.89(\mathrm{~d}, J=$ $\left.9.2 \mathrm{~Hz}, 6 \mathrm{H}, \mathrm{H}_{\mathrm{d}}\right), 2.58\left(\mathrm{~s}, 18 \mathrm{H}, \mathrm{CH}_{3}\right), 2.56\left(\mathrm{~s}, 18 \mathrm{H}, \mathrm{CH}_{3}\right), 2.36\left(\mathrm{~s}, 18 \mathrm{H}, \mathrm{CH}_{3}\right), 1.83(\mathrm{~s}$, $\left.18 \mathrm{H}, \mathrm{CH}_{3}\right), 1.65\left(\mathrm{~s}, 18 \mathrm{H}, \mathrm{CH}_{3}\right), 1.56\left(\mathrm{~s}, 18 \mathrm{H}, \mathrm{CH}_{3}\right)$ ppm. ${ }^{13} \mathrm{C}\left\{{ }^{1} \mathrm{H}\right\}$ NMR $(150 \mathrm{MHz}$, $\mathrm{CD}_{3} \mathrm{CN}$ ) $\delta=176.0$ (from $\mathrm{HMBC}, \mathrm{C}_{\mathrm{NHC}}$ ), 174.7 (from $\mathrm{HMBC}, \mathrm{C}_{\mathrm{NHC}}$ ), $170.6\left(\mathrm{C}_{\text {triazine}}\right.$ ), $169.5\left(\mathrm{C}_{\text {triazine }}\right), 141.7,140.7,140.5,136.8,135.8,135.4,135.3,135.1,135.0,131.5$, $131.1,130.9,130.8,130.7,130.3,130.2,130.1,129.9,129.7,129.5,128.7,128.6,127.4$ $\left(\mathrm{C}_{\mathrm{e}}\right), 127.2\left(\mathrm{C}_{\mathrm{b}}\right), 126.9\left(\mathrm{C}_{\mathrm{e}^{\prime}}\right), 126.0,125.1\left(\mathrm{C}_{\mathrm{c}}\right), 124.0\left(\mathrm{C}_{\mathrm{c}^{\prime}}\right), 119.6\left(\mathrm{C}_{\mathrm{d}}\right), 118.8\left(\mathrm{C}_{\mathrm{d}^{\prime}}\right)$, $115.1\left(\mathrm{C}_{\mathrm{f}}\right), 114.9\left(\mathrm{C}_{\mathrm{f}}\right), 21.48\left(\mathrm{CH}_{3}\right), 21.47\left(\mathrm{CH}_{3}\right), 18.4\left(\mathrm{CH}_{3}\right), 17.6\left(\mathrm{CH}_{3}\right), 17.2\left(\mathrm{CH}_{3}\right)$, $17.1\left(\mathrm{CH}_{3}\right)$ ppm. ESI-TOF MS: $m / z=1710.2998\left(\right.$ calcd for $\left\{\left[\mathrm{Ag}_{3}(\mathbf{1 b})_{2}\right]_{2}\left(\mathrm{PF}_{6}\right)_{3}\right\}^{3+}$ 1710.4100), 1246.4808 (calcd for $\left.\left\{\left[\mathrm{Ag}_{3}(\mathbf{1 b})_{2}\right]_{2}\left(\mathrm{PF}_{6}\right)_{2}\right\}^{4+} 1246.5645\right), 968.1995$ (calcd for $\left\{\left[\mathrm{Ag}_{3}(\mathbf{1 b})_{2}\right]_{2}\left(\mathrm{PF}_{6}\right)\right\}^{5+}$ 968.2619), 782.8387 (calcd for $\left.\left\{\left[\mathrm{Ag}_{3}(\mathbf{1 b})_{2}\right]_{2}\right\}^{6+} 782.8606\right)$. 

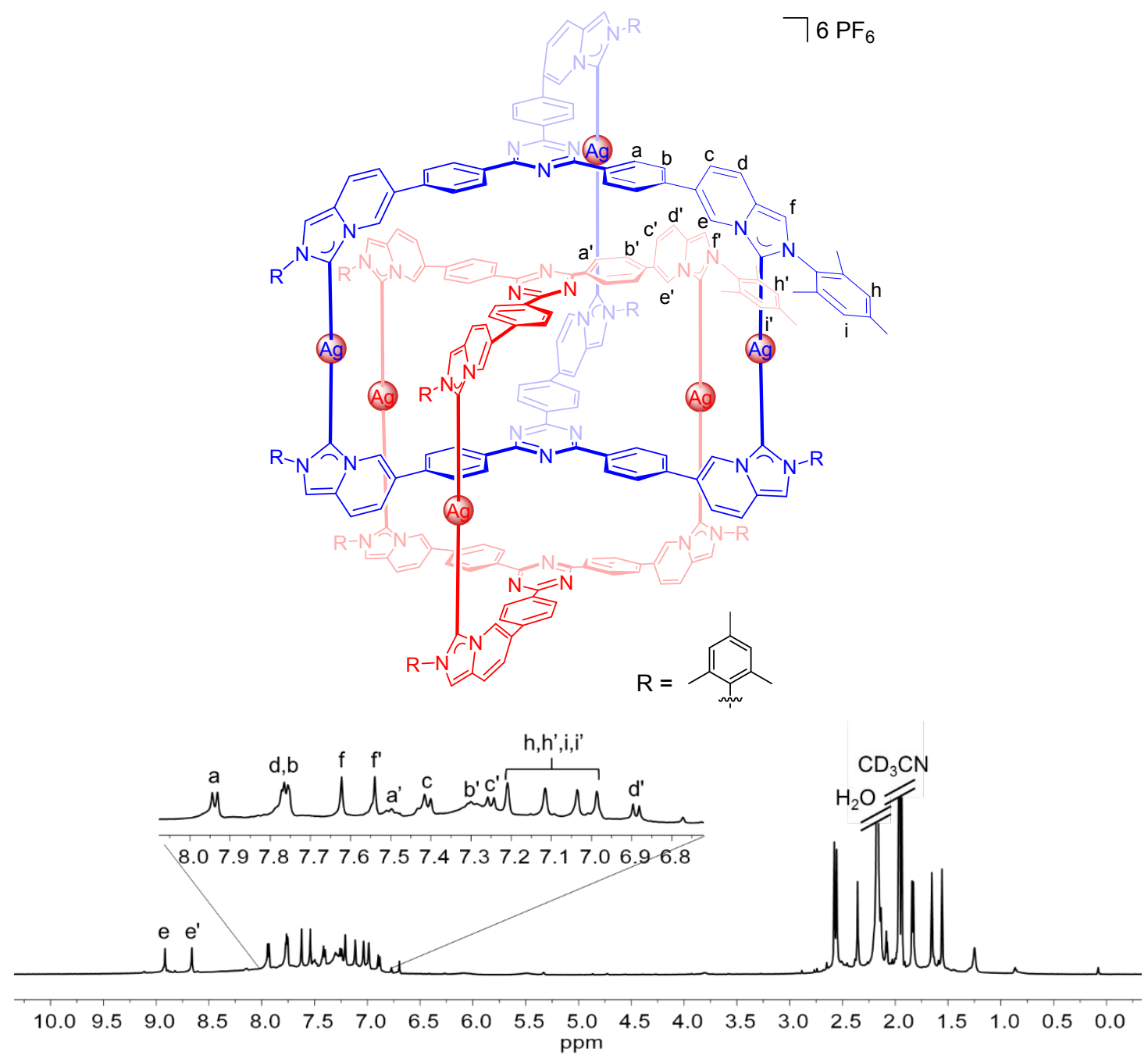

Figure S25. ${ }^{1} \mathrm{H}$ NMR spectrum $\left(600 \mathrm{MHz}, \mathrm{CD}_{3} \mathrm{CN}\right)$ of $\left[\mathrm{Ag}_{3}(\mathbf{1 b})_{2}\right]_{2}\left(\mathrm{PF}_{6}\right)_{6}$.

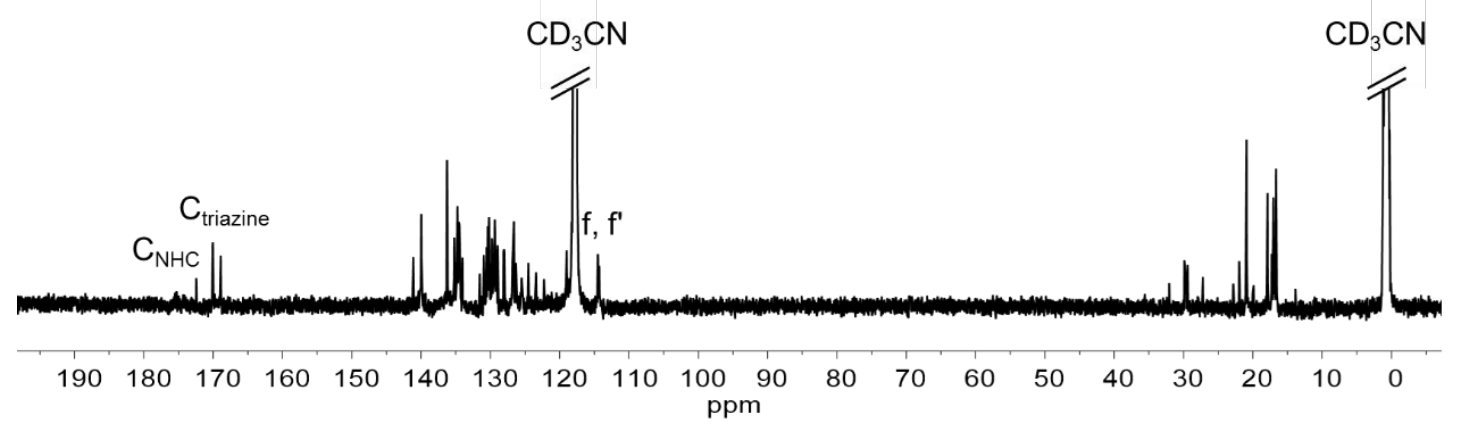

Figure S26. ${ }^{13} \mathrm{C}\left\{{ }^{1} \mathrm{H}\right\}$ NMR spectrum $\left(150 \mathrm{MHz}, \mathrm{CD}_{3} \mathrm{CN}\right)$ of $\left[\mathrm{Ag}_{3}(\mathbf{1 b})_{2}\right]_{2}\left(\mathrm{PF}_{6}\right)_{6}$. 


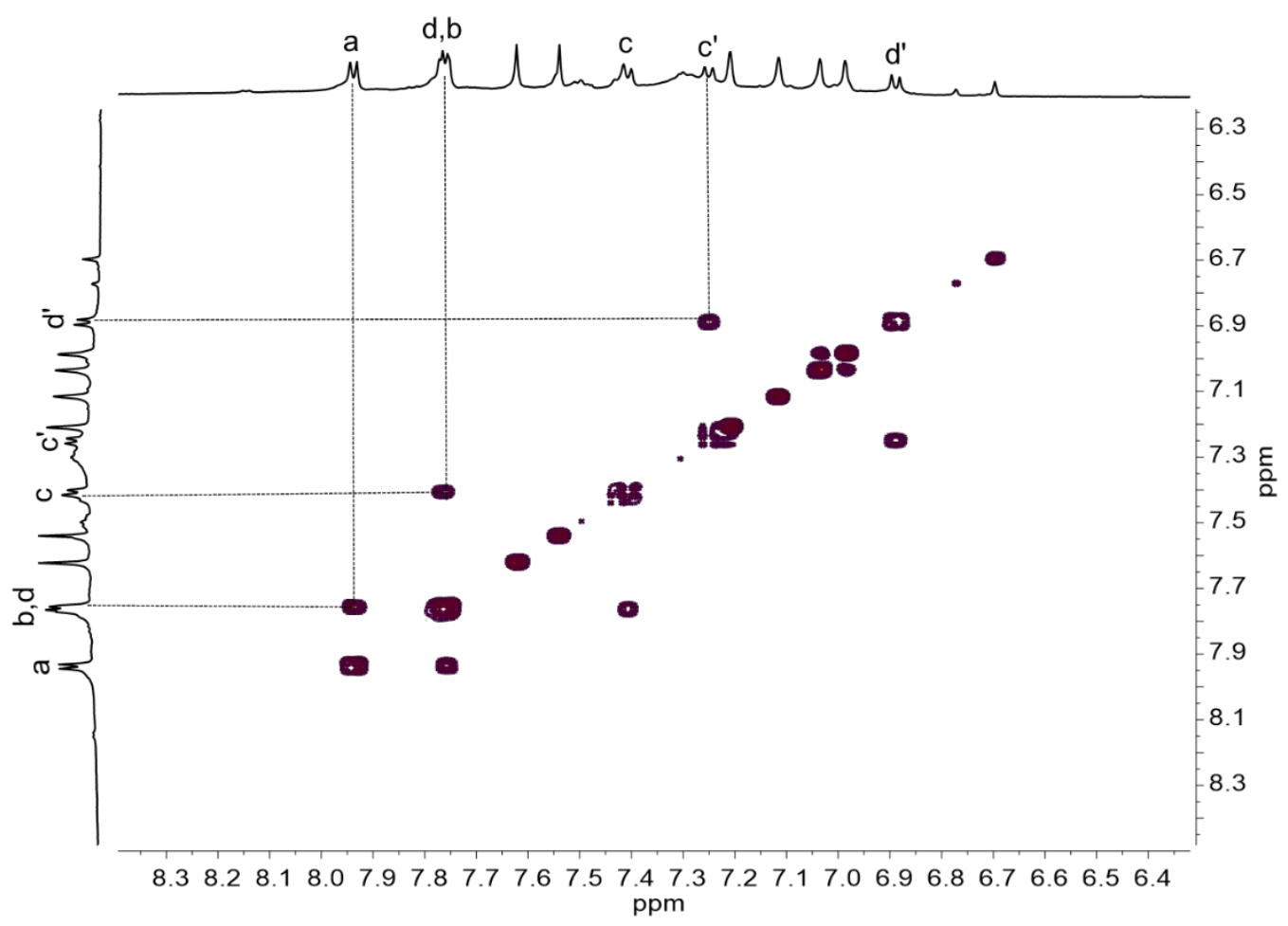

Figure S27. ${ }^{1} \mathrm{H}-{ }^{1} \mathrm{H}$ COSY spectrum $\left(600 \mathrm{MHz}, \mathrm{CD}_{3} \mathrm{CN}\right)$ of $\left[\mathrm{Ag}_{3}(\mathbf{1 b})_{2}\right]_{2}\left(\mathrm{PF}_{6}\right)_{6}$.

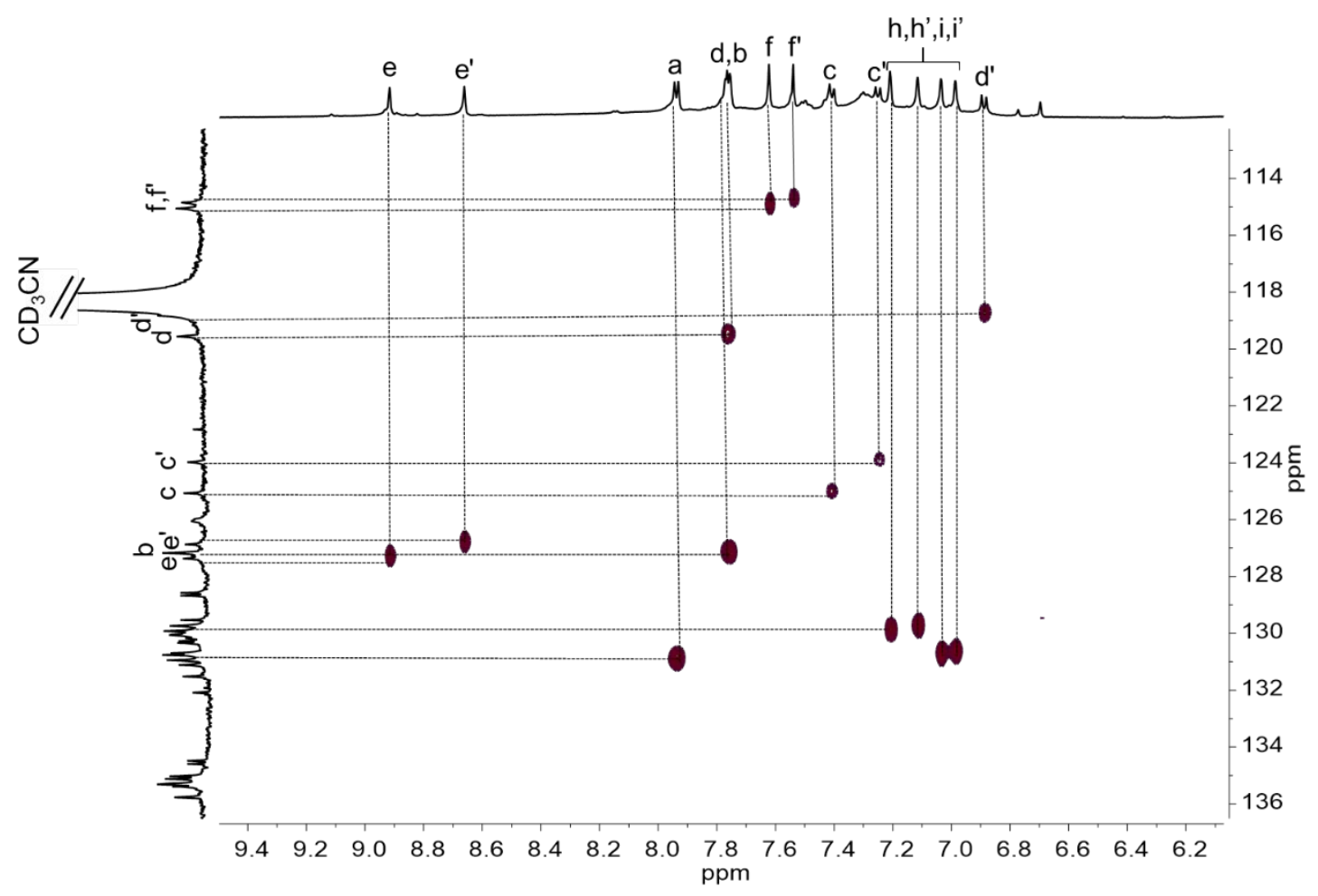

Figure S28. ${ }^{1} \mathrm{H}^{13} \mathrm{C}$ HSQC spectrum $\left(600 \mathrm{MHz}, \mathrm{CD}_{3} \mathrm{CN}\right)$ of $\left[\mathrm{Ag}_{3}(\mathbf{1 b})_{2}\right]_{2}\left(\mathrm{PF}_{6}\right)_{6}$. 


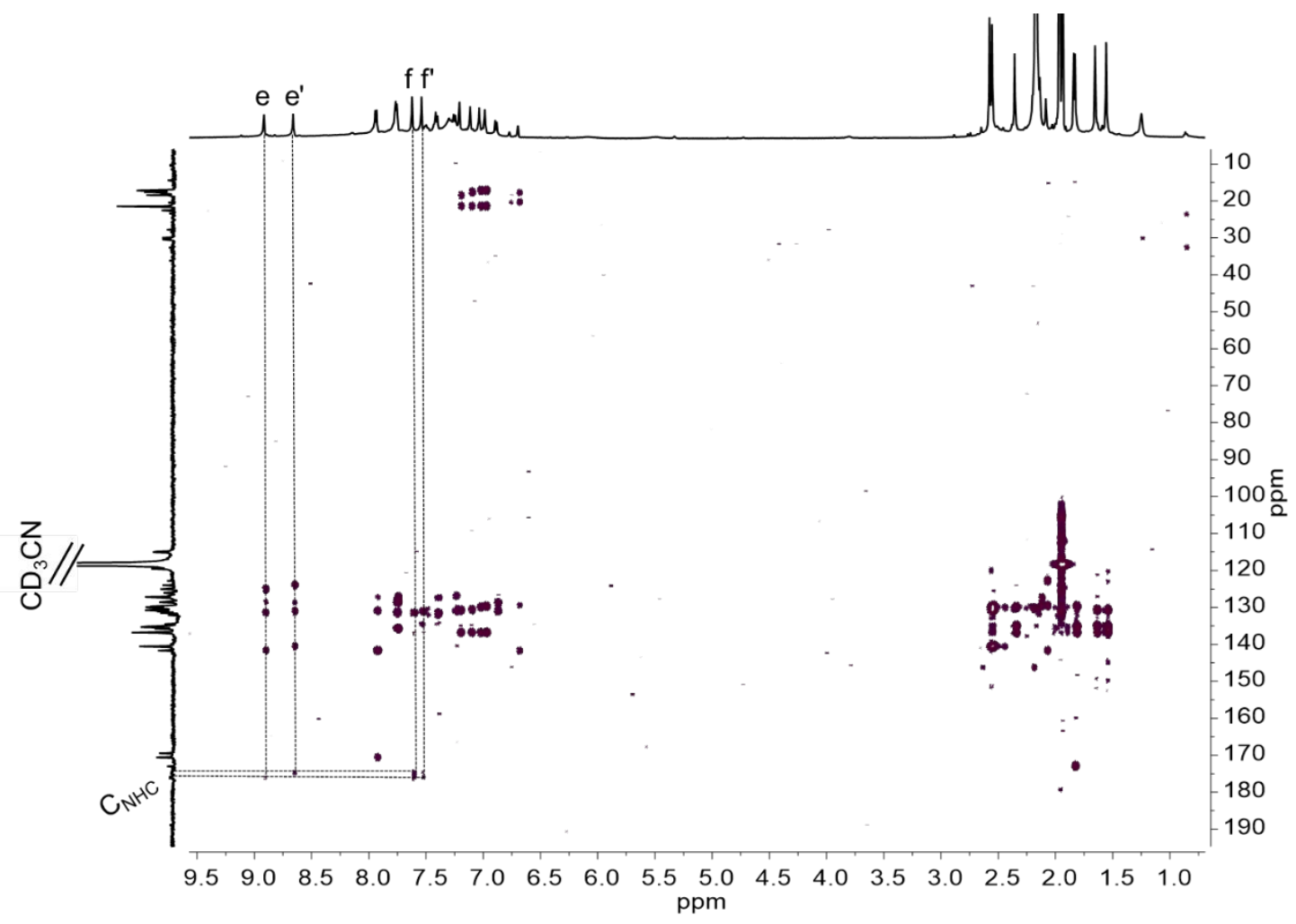

Figure S29. ${ }^{1} \mathrm{H}-{ }^{13} \mathrm{C}$ HMBC spectrum $\left(600 \mathrm{MHz}, \mathrm{CD}_{3} \mathrm{CN}\right)$ of $\left[\mathrm{Ag}_{3}(\mathbf{1 b})_{2}\right]_{2}\left(\mathrm{PF}_{6}\right)_{6}$.

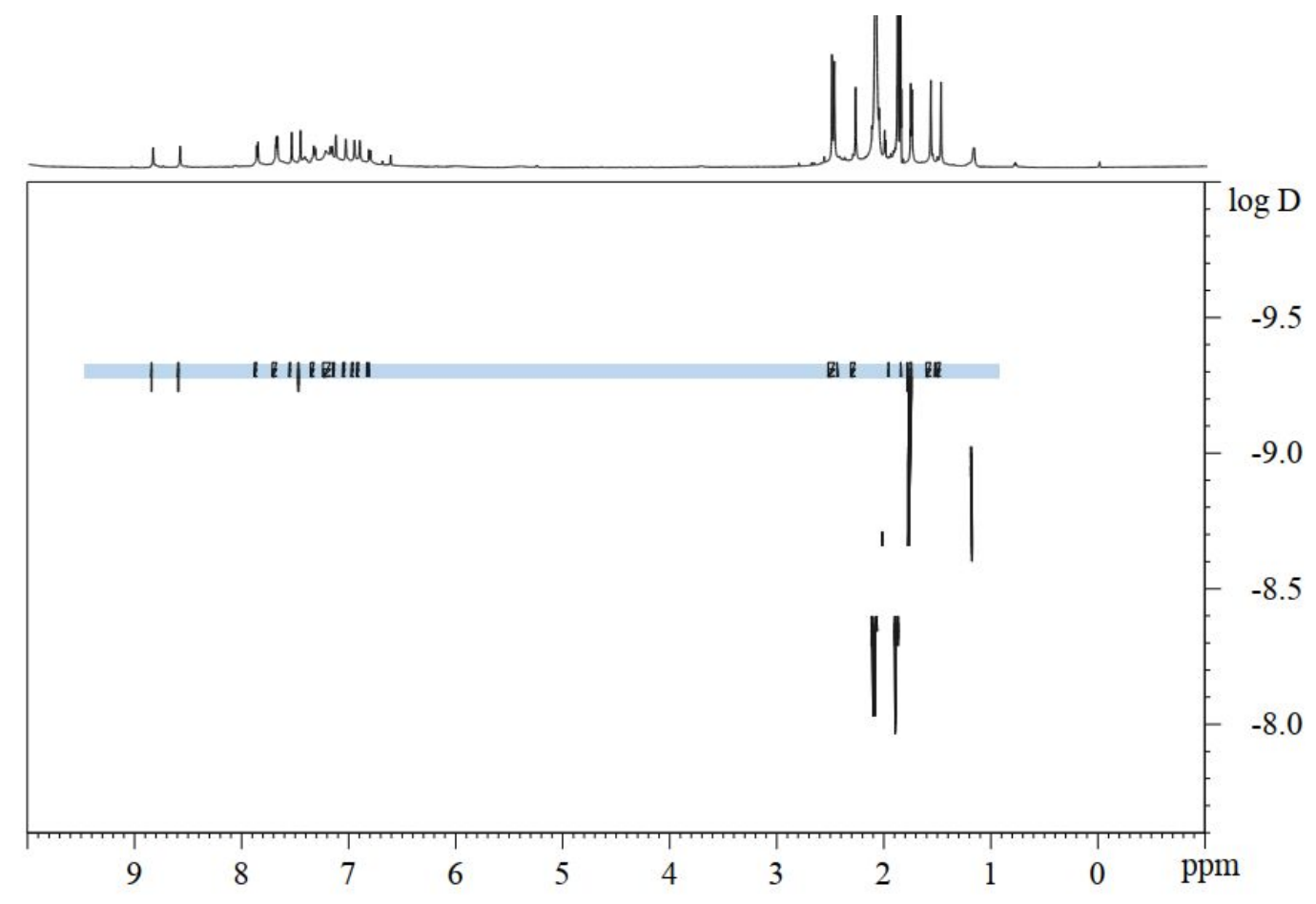

Figure S30. ${ }^{1} \mathrm{H}$ DOSY spectrum $\left(600 \mathrm{MHz}, \mathrm{CD}_{3} \mathrm{CN}\right)$ of $\left[\mathrm{Ag}_{3}(\mathbf{1 b})_{2}\right]_{2}\left(\mathrm{PF}_{6}\right)_{6} \cdot[\mathrm{D}=4.467$

$\left.\times 10^{-10} \mathrm{~m}^{2} \mathrm{~s}^{-1}(\log \mathrm{D}=-9.35)\right]$. 


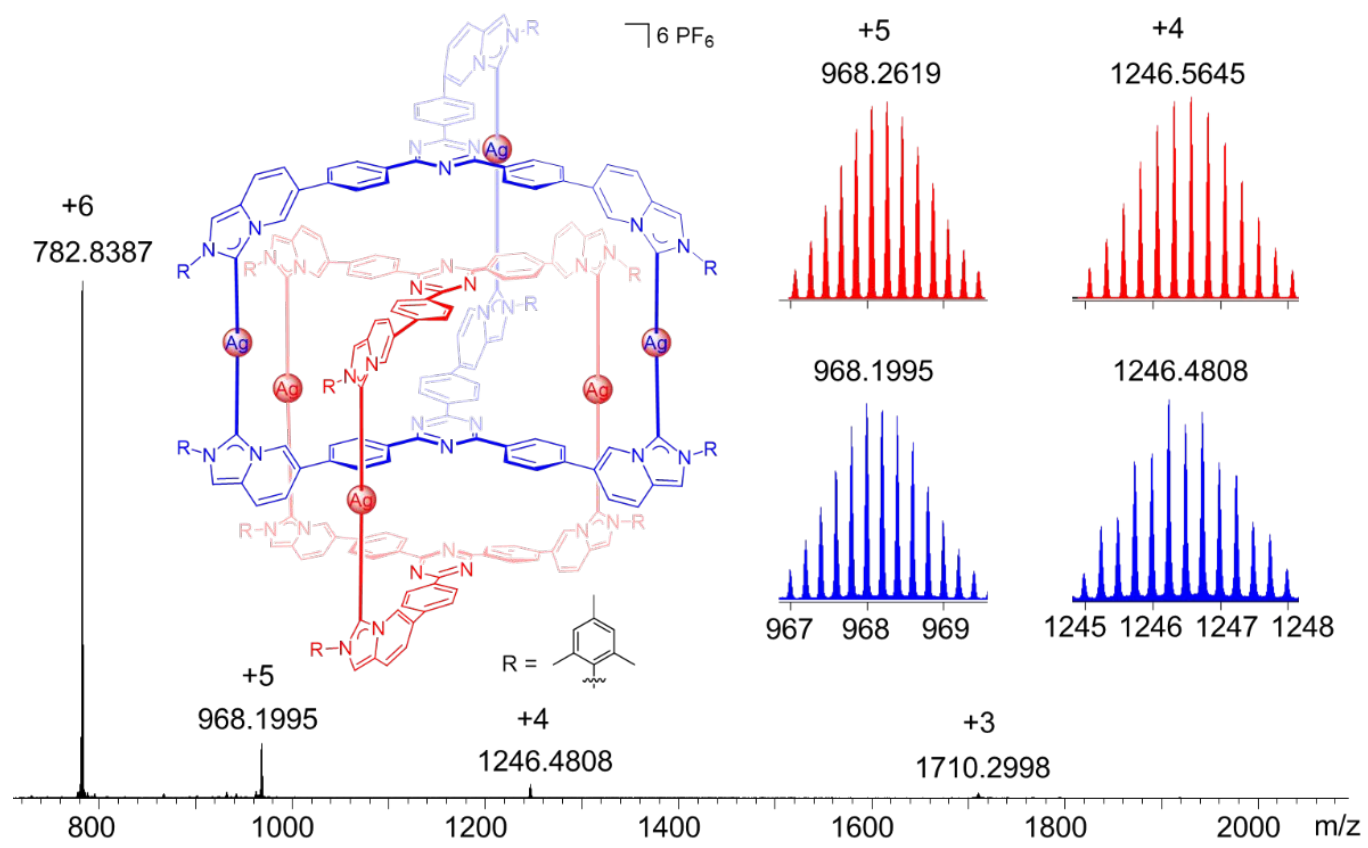

Figure S31. ESI-TOF mass spectrum of $\left[\mathrm{Ag}_{3}(\mathbf{1 b})_{2}\right]_{2}\left(\mathrm{PF}_{6}\right)_{6}$ with isotope distribution for selected peaks (experimental in blue, calculated in red, $\mathrm{PF}_{6}^{-}$counterions).

\subsection{Synthesis of complex $\left[\operatorname{Ag}_{3}(1 \mathrm{c})_{2}\right]_{2}\left(\mathrm{PF}_{6}\right)_{6}$}

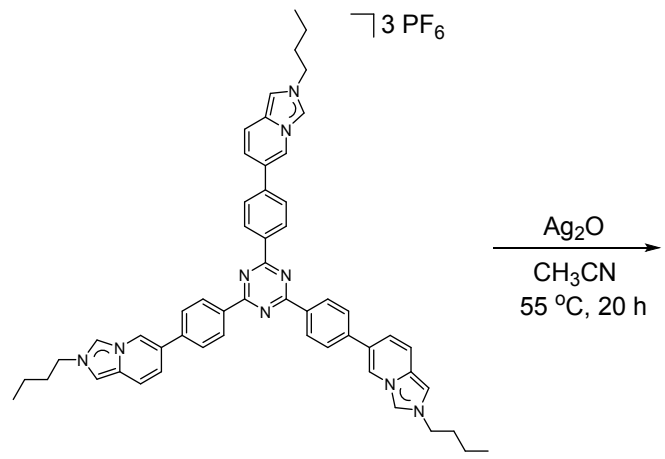

$\mathrm{H}_{3}-1 \mathbf{c}\left(\mathrm{PF}_{6}\right)_{3}$

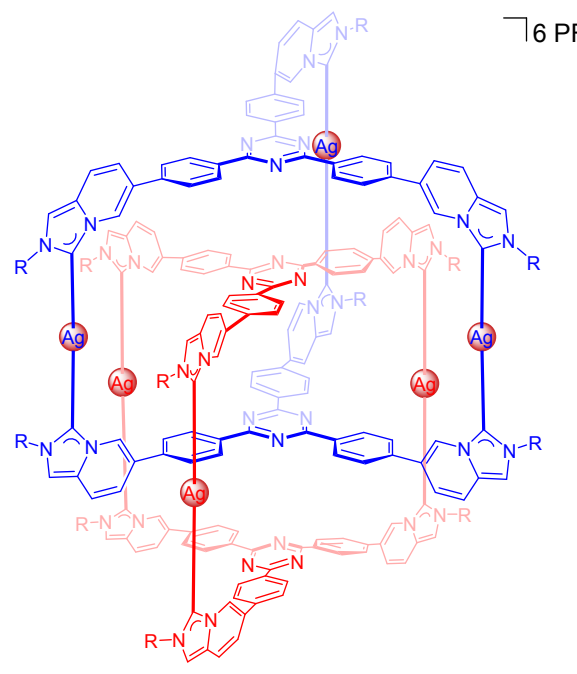

$\left[\mathrm{Ag}_{3}(1 \mathrm{c})_{2}\right]_{2}\left(\mathrm{PF}_{6}\right)_{6}$ $\mathrm{R}=-\left(\mathrm{CH}_{2}\right)_{3} \mathrm{CH}_{3}$

Scheme S4. Synthesis of Complex $\left[\mathrm{Ag}_{3}(1 \mathrm{c})_{2}\right]_{2}\left(\mathrm{PF}_{6}\right)_{6}$.

Complex $\left[\mathrm{Ag}_{3}(\mathbf{1 c})_{2}\right]_{2}\left(\mathrm{PF}_{6}\right)_{6}$ was prepared from $\mathrm{H}_{3}-\mathbf{1 c}\left(\mathrm{PF}_{6}\right)_{3}(30 \mathrm{mg}, 0.024 \mathrm{mmol})$ and $\mathrm{Ag}_{2} \mathrm{O}$ (25 mg, $\left.0.108 \mathrm{mmol}\right)$. Yield: $23 \mathrm{mg}(0.0048 \mathrm{mmol}, 80 \%) .{ }^{1} \mathrm{H}$ NMR (600 MHz, $\left.\mathrm{CD}_{3} \mathrm{CN}\right) \delta=8.62\left(\mathrm{~s}, 6 \mathrm{H}, \mathrm{H}_{\mathrm{e}}\right), 8.26\left(\mathrm{~s}, 6 \mathrm{H}, \mathrm{H}_{\mathrm{e}}\right), 8.19\left(\right.$ br s, $\left.12 \mathrm{H}, \mathrm{H}_{\mathrm{a}}\right), 7.57\left(\mathrm{~s}, 6 \mathrm{H}, \mathrm{H}_{\mathrm{f}}\right)$, 
$7.54\left(\mathrm{~d}, J=9.2 \mathrm{~Hz}, 6 \mathrm{H}, \mathrm{H}_{\mathrm{d}}\right), 7.47\left(\mathrm{~s}, 6 \mathrm{H}, \mathrm{H}_{\mathrm{f}^{\prime}}\right), 7.35-7.28\left(\mathrm{~m}, 36 \mathrm{H}, \mathrm{H}_{\mathrm{a}^{\prime}, \mathrm{b}, \mathrm{b}^{\prime}}\right), 7.10(\mathrm{~d}, J=$ $\left.9.2 \mathrm{~Hz}, 6 \mathrm{H}, \mathrm{H}_{\mathrm{d}^{\prime}}\right), 7.07\left(\mathrm{~d}, J=9.2 \mathrm{~Hz}, 6 \mathrm{H}, \mathrm{H}_{\mathrm{c}}\right), 6.78\left(\mathrm{~d}, J=9.2 \mathrm{~Hz}, 6 \mathrm{H}, \mathrm{H}_{\mathrm{c}}\right), 4.63(\mathrm{t}, J=$ $\left.7.2 \mathrm{~Hz}, 12 \mathrm{H}, \mathrm{H}_{\mathrm{h}}\right), 4.30-4.25\left(\mathrm{~m}, 6 \mathrm{H}, \mathrm{H}_{\mathrm{h}}\right), 4.15-4.10\left(\mathrm{~m}, 6 \mathrm{H}, \mathrm{H}_{\mathrm{h}}\right), 2.05-1.96(\mathrm{~m}, 24 \mathrm{H}$, $\left.\mathrm{H}_{\mathrm{i}, \mathrm{i}^{\prime}}\right), 1.47-1.34\left(\mathrm{~m}, 24 \mathrm{H}, \mathrm{H}_{\mathrm{j}, \mathrm{j}^{\prime}}\right), 1.03-1.00\left(\mathrm{~m}, 36 \mathrm{H}, \mathrm{H}_{\mathrm{k}, \mathrm{k}^{\prime}}\right) \mathrm{ppm} .{ }^{13} \mathrm{C}\left\{{ }^{1} \mathrm{H}\right\} \mathrm{NMR}(150$ $\left.\mathrm{MHz}, \mathrm{CD}_{3} \mathrm{CN}\right) \delta=172.9\left(\mathrm{C}_{\mathrm{NHC}}\right), 172.0\left(\mathrm{C}_{\mathrm{NHC}}\right), 170.7\left(\mathrm{C}_{\text {triazine }}\right), 169.8\left(\mathrm{C}_{\text {triazine }}\right), 141.6$, $140.7,135.7,135.4,130.8,130.7,130.66,130.63,130.2,129.7,128.9,128.3,127.2$ $\left(\mathrm{C}_{\mathrm{e}}\right), 125.5\left(\mathrm{C}_{\mathrm{e}}\right), 124.7\left(\mathrm{C}_{\mathrm{c}}\right), 124.5\left(\mathrm{C}_{\mathrm{c}^{\prime}}\right), 119.2\left(\mathrm{C}_{\mathrm{d}}\right), 119.1\left(\mathrm{C}_{\mathrm{d}^{\prime}}\right), 113.8\left(\mathrm{C}_{\mathrm{f}}\right), 113.1\left(\mathrm{C}_{\mathrm{f}^{\prime}}\right)$, $54.5\left(\mathrm{C}_{\mathrm{h}}\right), 54.2\left(\mathrm{C}_{\mathrm{h}^{\prime}}\right), 35.2\left(\mathrm{C}_{\mathrm{i}}\right), 35.0\left(\mathrm{C}_{\mathrm{i}^{\prime}}\right), 20.9\left(\mathrm{C}_{\mathrm{j}}\right), 20.7\left(\mathrm{C}_{\mathrm{j}^{\prime}}\right), 14.1\left(\mathrm{C}_{\mathrm{k}}\right), 14.0\left(\mathrm{C}_{\mathrm{k}^{\prime}}\right)$ ppm. ESI-TOF MS: $m / z=1462.0123$ (calcd for $\left.\left\{\left[\mathrm{Ag}_{3}(\mathbf{1} \mathbf{c})_{2}\right]_{2}\left(\mathrm{PF}_{6}\right)_{3}\right\}^{3+} 1462.0121\right)$, 1060.2689 (calcd for $\left.\left\{\left[\mathrm{Ag}_{3}(\mathbf{1} \mathbf{c})_{2}\right]_{2}\left(\mathrm{PF}_{6}\right)_{2}\right\}^{4+} 1060.2679\right)$, 819.2219 (calcd for $\left\{\left[\mathrm{Ag}_{3}\left(\mathbf{1 c}_{2}\right]_{2}\left(\mathrm{PF}_{6}\right)\right\}^{5+}\right.$ 819.2216), 658.5237 (calcd for $\left\{\left[\mathrm{Ag}_{3}(\mathbf{1} \mathbf{c})_{2}\right]_{2}\right\}^{6+}$ 658.5239).

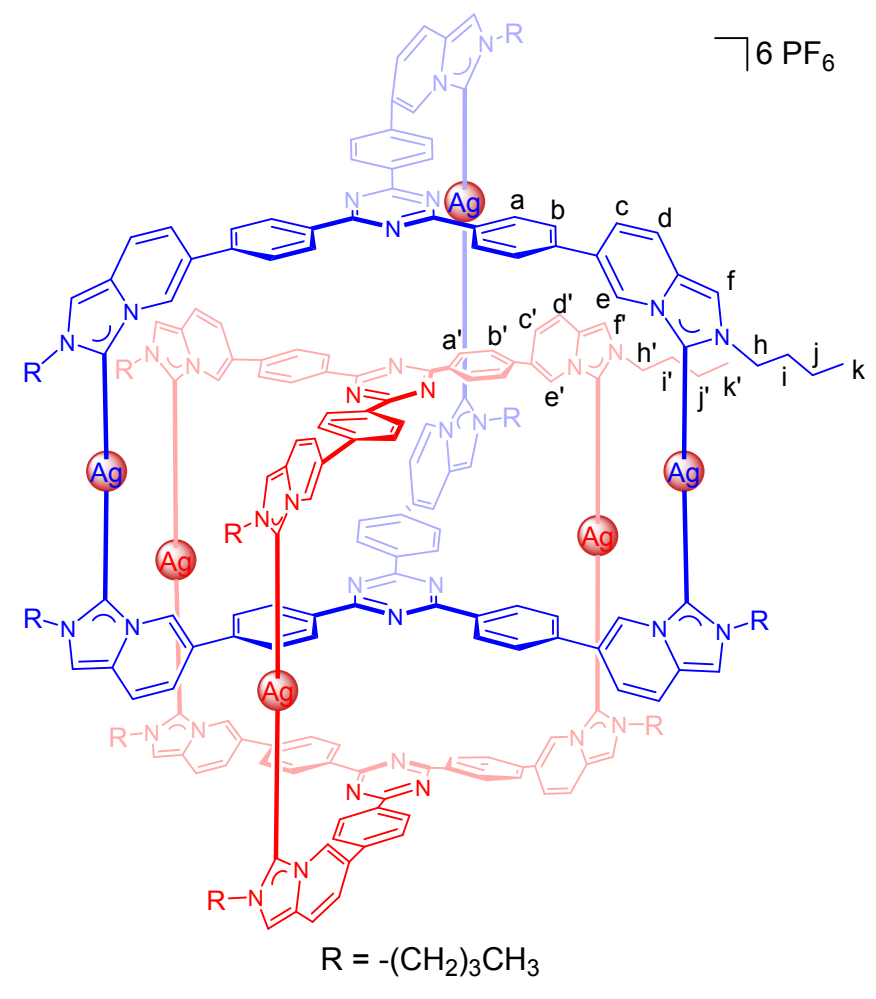




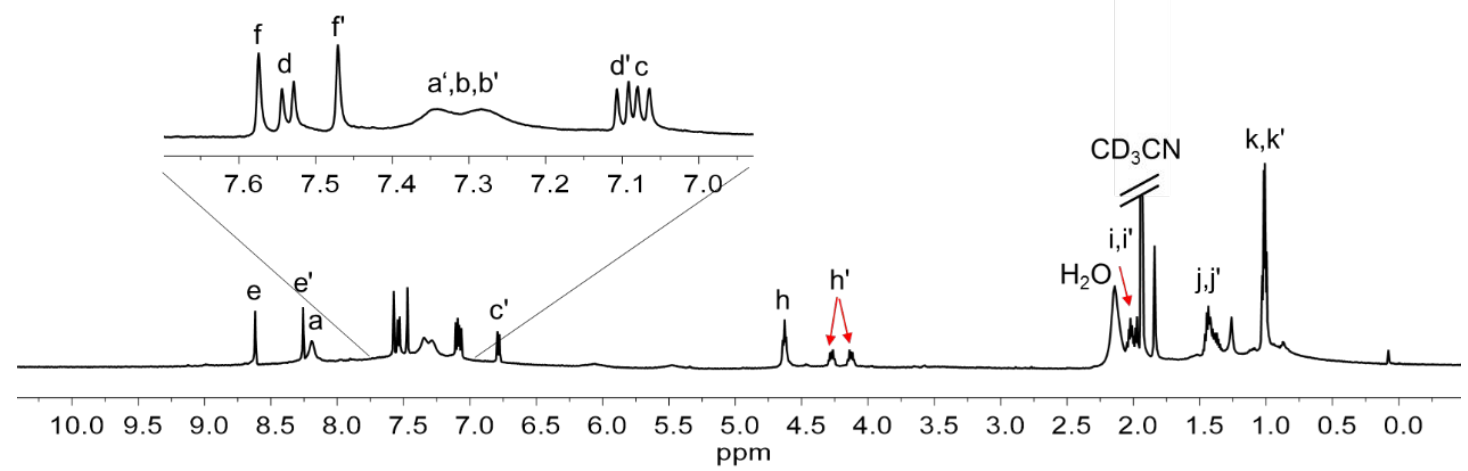

Figure S32. ${ }^{1} \mathrm{H}$ NMR spectrum $\left(600 \mathrm{MHz}, \mathrm{CD}_{3} \mathrm{CN}\right)$ of $\left[\mathrm{Ag}_{3}(\mathbf{1 c})_{2}\right]_{2}\left(\mathrm{PF}_{6}\right)_{6}$.

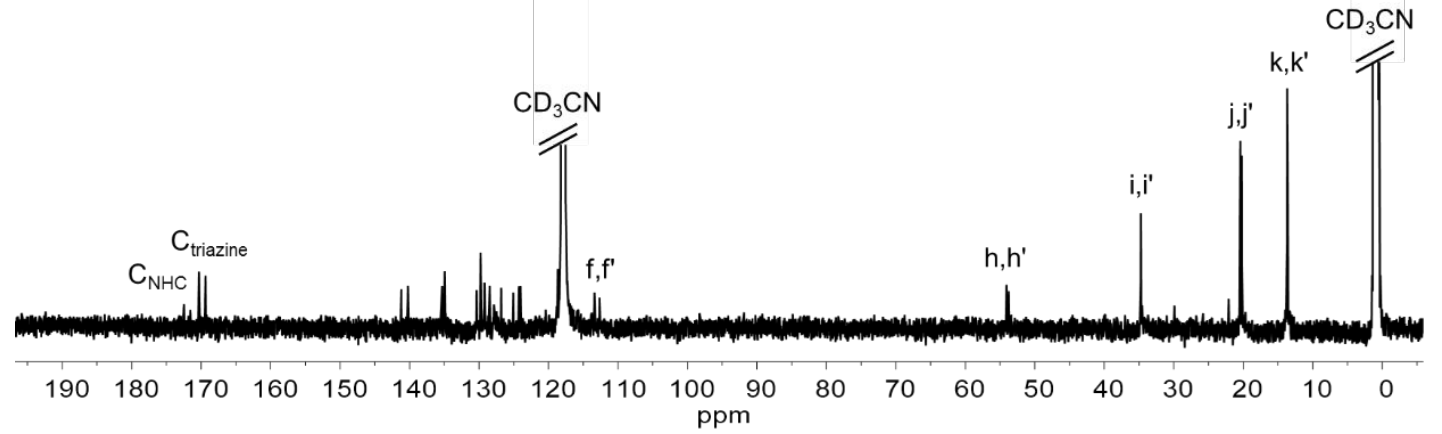

FigureS 33. ${ }^{13} \mathrm{C}\left\{{ }^{1} \mathrm{H}\right\}$ NMR spectrum $\left(150 \mathrm{MHz}, \mathrm{CD}_{3} \mathrm{CN}\right)$ of $\left[\mathrm{Ag}_{3}(\mathbf{1 c})_{2}\right]_{2}\left(\mathrm{PF}_{6}\right)_{6}$.

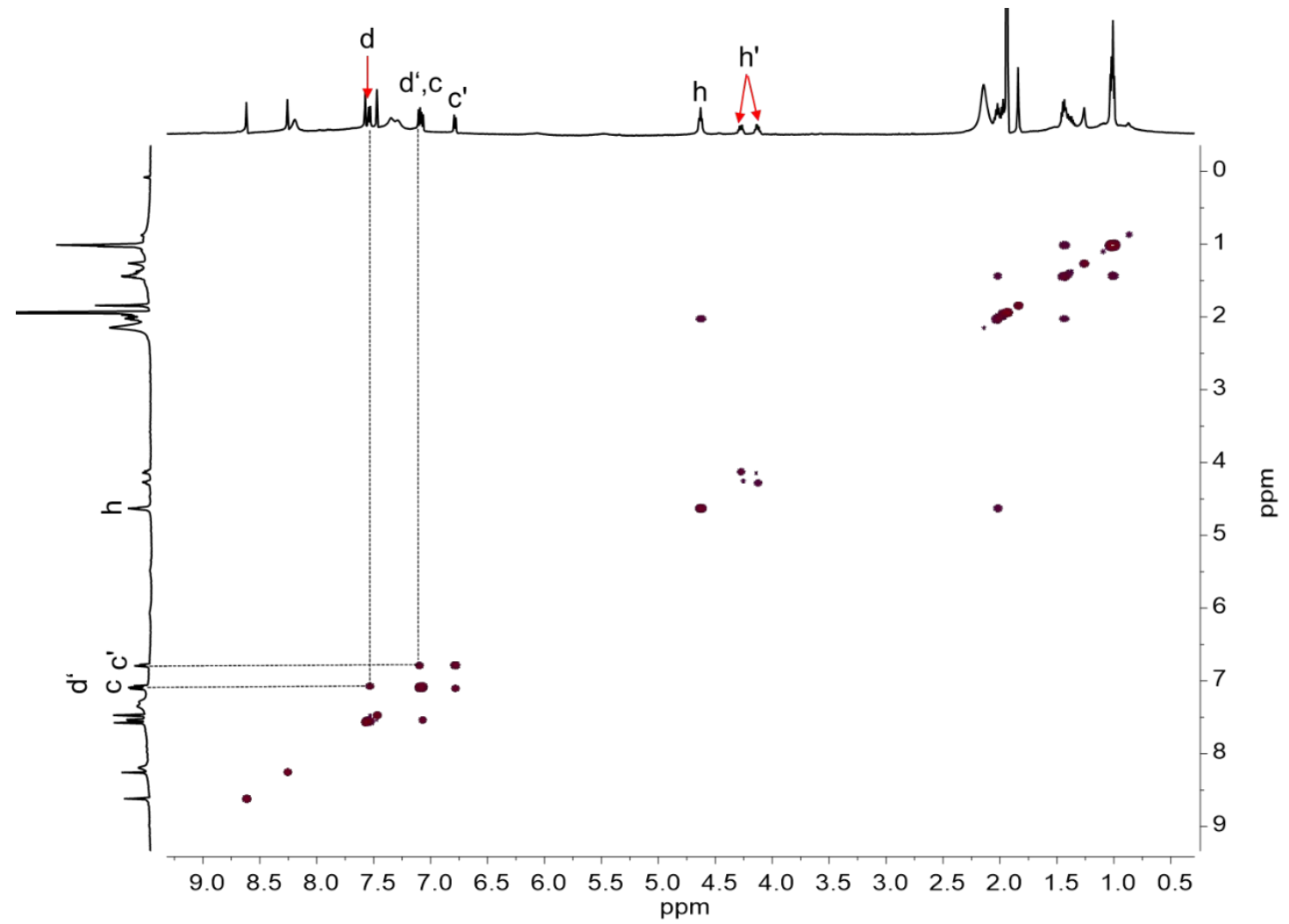

Figure S34. ${ }^{1} \mathrm{H}-{ }^{1} \mathrm{H}$ COSY spectrum $\left(600 \mathrm{MHz}, \mathrm{CD}_{3} \mathrm{CN}\right)$ of $\left[\mathrm{Ag}_{3}(\mathbf{1 c})_{2}\right]_{2}\left(\mathrm{PF}_{6}\right)_{6}$. 


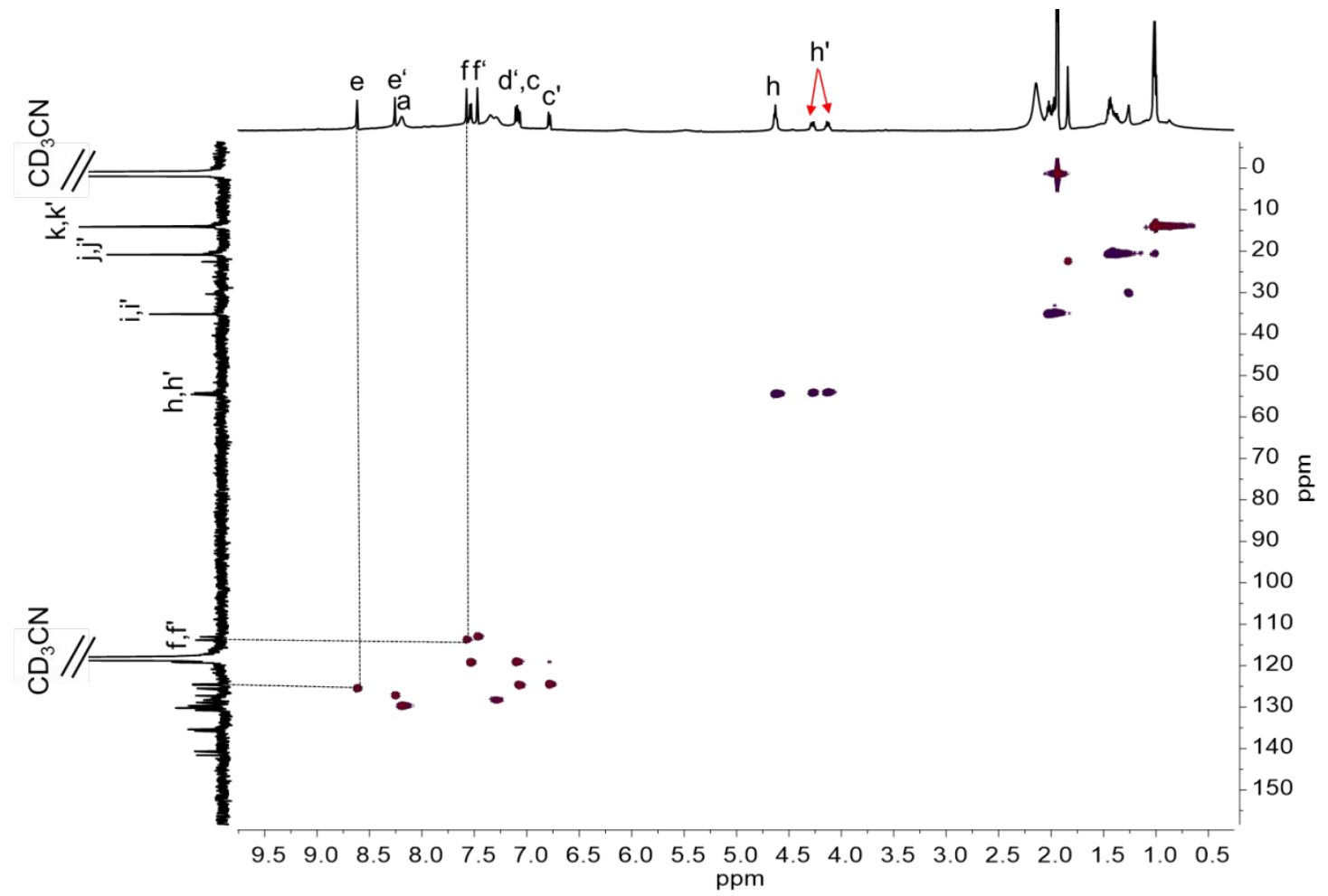

Figure S35. ${ }^{1} \mathrm{H}-{ }^{13} \mathrm{C}$ HSQC spectrum $\left(600 \mathrm{MHz}, \mathrm{CD}_{3} \mathrm{CN}\right)$ of $\left[\mathrm{Ag}_{3}(\mathbf{1 c})_{2}\right]_{2}\left(\mathrm{PF}_{6}\right)_{6}$.

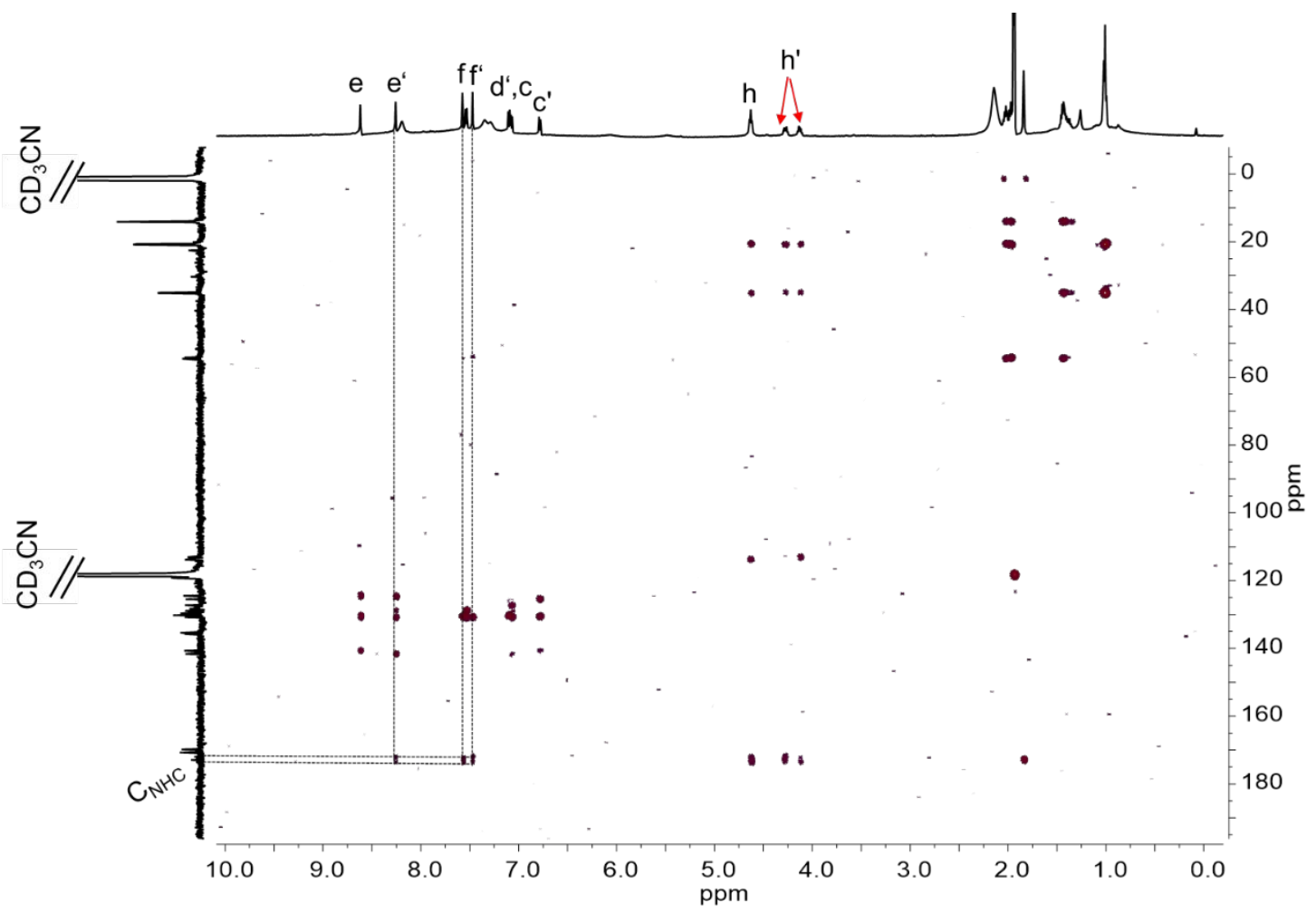

Figure S36. ${ }^{1} \mathrm{H}-{ }^{13} \mathrm{C}$ HMBC spectrum $\left(600 \mathrm{MHz}, \mathrm{CD}_{3} \mathrm{CN}\right)$ of $\left[\mathrm{Ag}_{3}(\mathbf{1 c})_{2}\right]_{2}\left(\mathrm{PF}_{6}\right)_{6}$. 


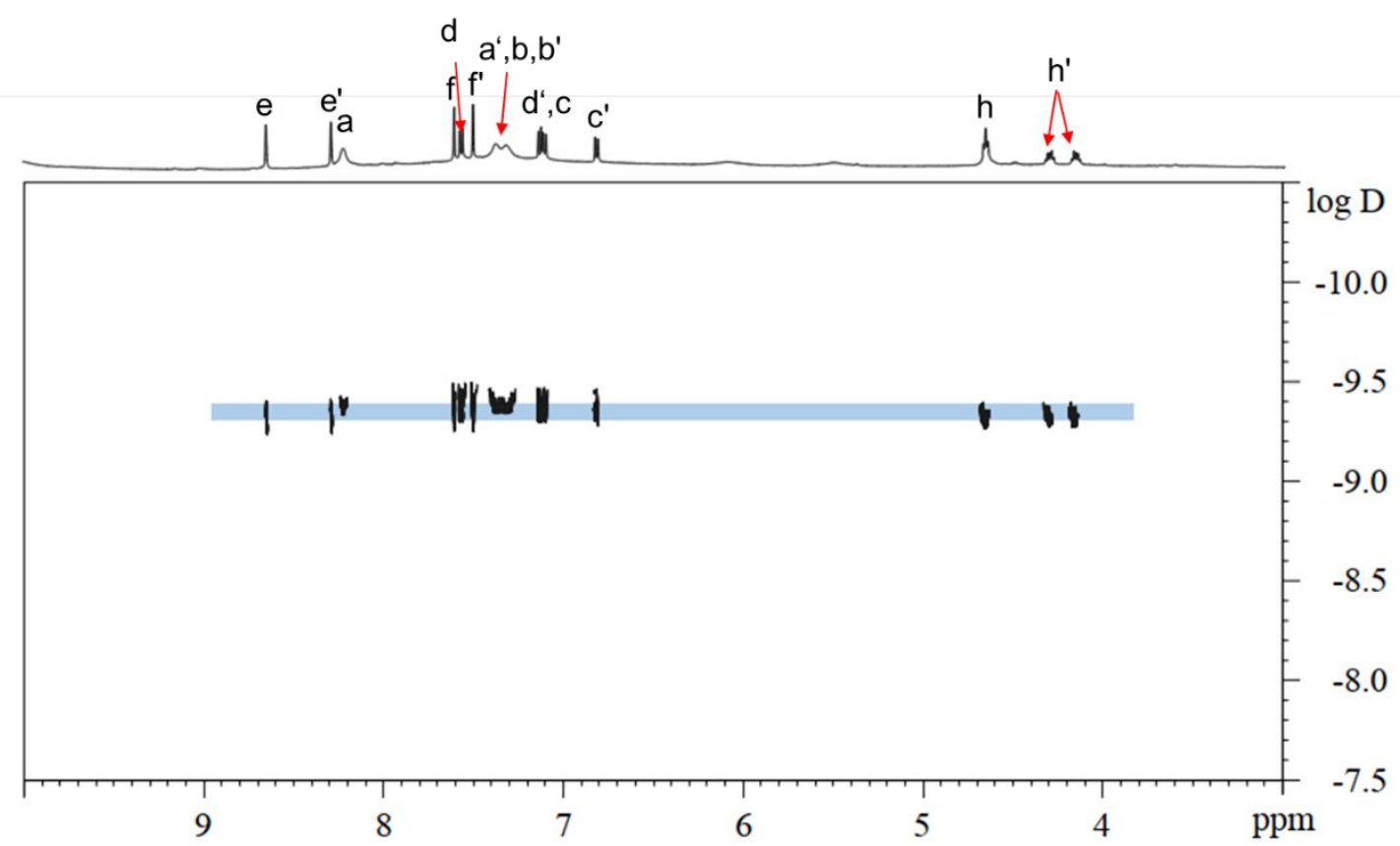

Figure S37. ${ }^{1} \mathrm{H}$ DOSY spectrum $\left(600 \mathrm{MHz}, \mathrm{CD}_{3} \mathrm{CN}\right)$ of $\left[\mathrm{Ag}_{3}(\mathbf{1 c})_{2}\right]_{2}\left(\mathrm{PF}_{6}\right)_{6} \cdot[\mathrm{D}=4.589$

$\left.\times 10^{-10} \mathrm{~m}^{2} \mathrm{~s}^{-1}(\log \mathrm{D}=-9.34)\right]$.

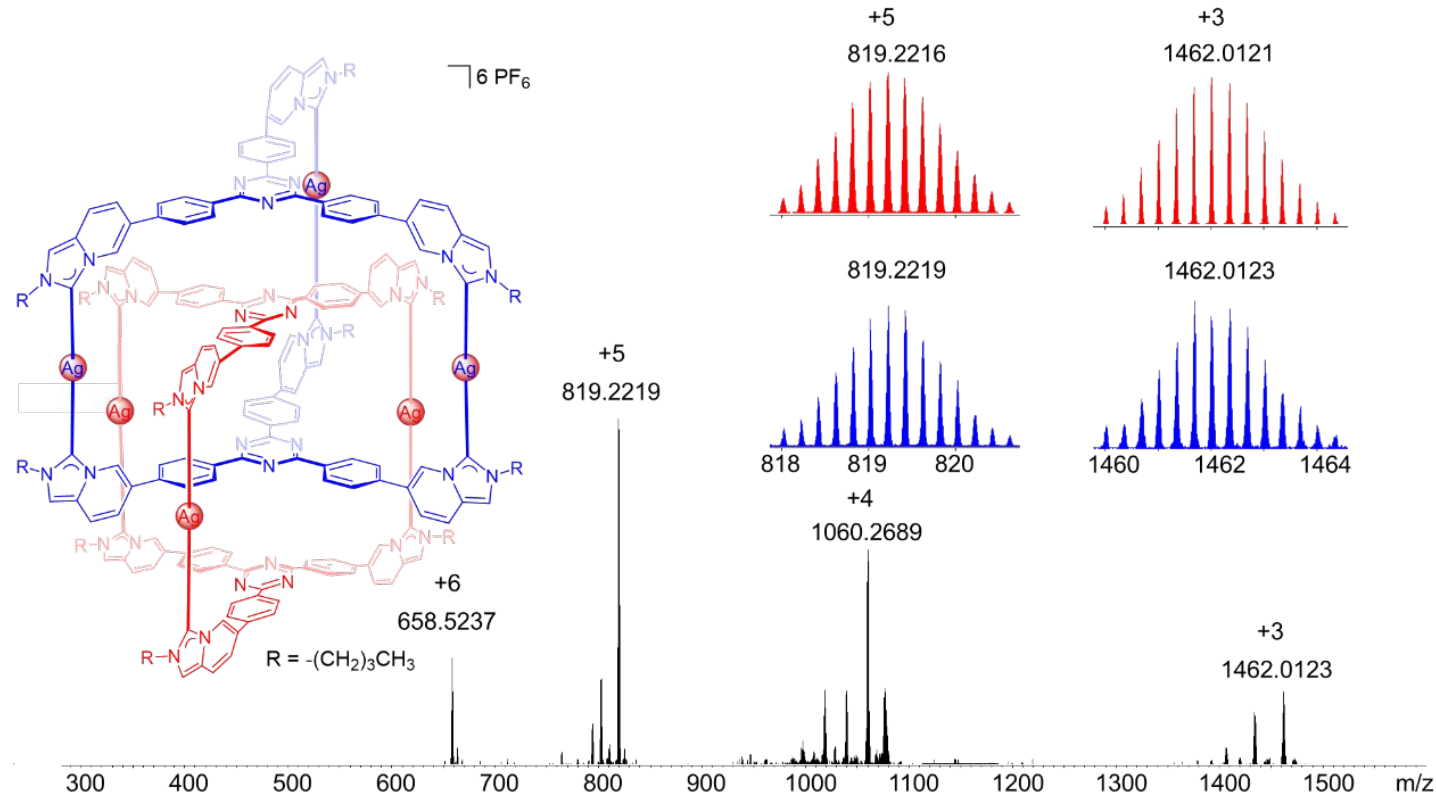

Figure S38. ESI-TOF mass spectrum of $\left[\mathrm{Ag}_{3}(\mathbf{1 c})_{2}\right]_{2}\left(\mathrm{PF}_{6}\right)_{6}$ with isotope distribution for selected peaks (experimental in blue, calculated in red, $\mathrm{PF}_{6}^{-}$counterions). 


\section{Synthesis of complexes $\left[\mathrm{Au}_{3}(\mathrm{~L})_{2}\right]_{2}\left(\mathrm{PF}_{6}\right)_{6}(\mathrm{~L}=1 \mathrm{~b}, 1 \mathrm{c})$}

\section{General synthetic procedure of complexes $\left[\mathrm{Au}_{3}(\mathrm{~L})_{2}\right]_{2}\left(\mathrm{PF}_{6}\right)_{6}(\mathrm{~L}=1 \mathrm{~b}, 1 \mathrm{c})$}

To a solution of $\left[\mathrm{Ag}_{3}(\mathbf{L})_{2}\right]_{2}\left(\mathrm{PF}_{6}\right)_{6}$ (in $20 \mathrm{~mL}$ of $\mathrm{CH}_{3} \mathrm{CN}$ ) was added 6 equivalents of $[\mathrm{AuCl}(\mathrm{THT})](\mathrm{THT}=$ tetrahydrothiophene $)$ under a nitrogen atmosphere. The reaction mixture was stirred at ambient temperature for $24 \mathrm{~h}$ and then slowly filtered through a pad of Celite until a clear filtrate was obtained. The filtrate was concentrated to $2 \mathrm{~mL}$, and diethyl ether $(20 \mathrm{~mL})$ was added. A purple precipitate formed, which was isolated by filtration, washed with diethyl ether and dried in vacuo to afford the complexes of type $\left[\mathrm{Au}_{3}(\mathbf{L})_{2}\right]_{2}\left(\mathrm{PF}_{6}\right)_{6}(\mathbf{L}=\mathbf{1 b}, \mathbf{1 c})$.

\subsection{Synthesis of complex $\left[\mathrm{Au}_{3}(1 \mathrm{~b})_{2}\right]_{2}\left(\mathrm{PF}_{6}\right)_{6}$}
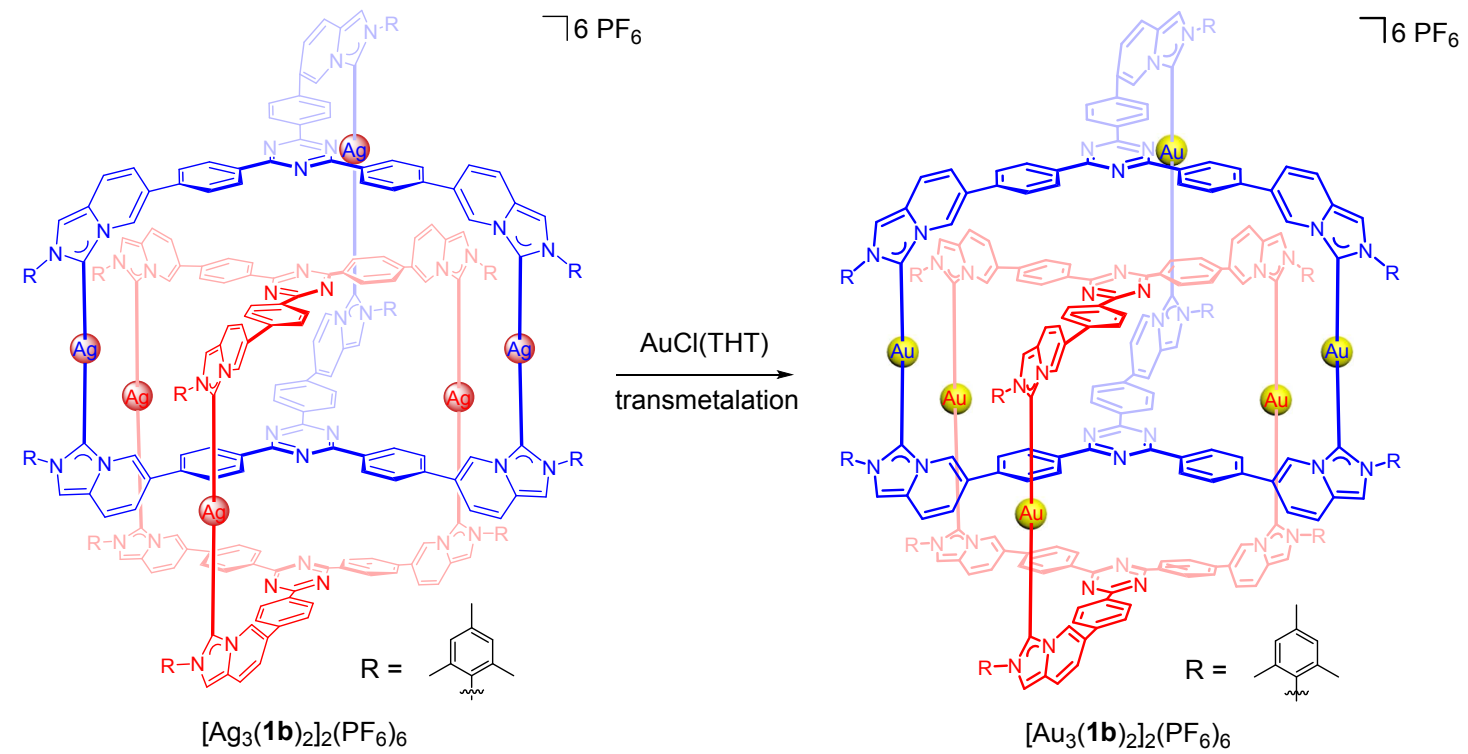

Scheme S5. Synthesis of Complex $\left[\mathrm{Au}_{3}(\mathbf{1 b})_{2}\right]_{2}\left(\mathrm{PF}_{6}\right)_{6}$.

Complex $\left[\mathrm{Au}_{3}(\mathbf{1 b})_{2}\right]_{2}\left(\mathrm{PF}_{6}\right)_{6}$ was prepared from $\left[\mathrm{Ag}_{3}(\mathbf{1} \mathbf{b})_{2}\right]_{2}\left(\mathrm{PF}_{6}\right)_{6}(30 \mathrm{mg}, 0.005 \mathrm{mmol})$ and $\mathrm{AuCl}(\mathrm{THT})(11 \mathrm{mg}, 0.03 \mathrm{mmol})$. Yield: $24 \mathrm{mg}(0.004 \mathrm{mmol}, 80 \%) .{ }^{1} \mathrm{H}$ NMR (600 $\left.\mathrm{MHz}, \mathrm{CD}_{3} \mathrm{CN}\right) \delta=9.01\left(\mathrm{~s}, 6 \mathrm{H}, \mathrm{H}_{\mathrm{e}}\right), 8.81\left(\mathrm{~s}, 6 \mathrm{H}, \mathrm{H}_{\mathrm{e}}\right), 7.91\left(\mathrm{~d}, J=7.7 \mathrm{~Hz}, 12 \mathrm{H}, \mathrm{H}_{\mathrm{a}}\right)$, $7.80\left(\mathrm{~d}, J=9.2 \mathrm{~Hz}, 6 \mathrm{H}, \mathrm{H}_{\mathrm{d}}\right), 7.78\left(\mathrm{~d}, J=7.7 \mathrm{~Hz}, 12 \mathrm{H}, \mathrm{H}_{\mathrm{b}}\right), 7.60\left(\mathrm{~s}, 6 \mathrm{H}, \mathrm{H}_{\mathrm{f}}\right), 7.56(\mathrm{~s}, 6 \mathrm{H}$, 
$\left.\mathrm{H}_{\mathrm{f}}\right), 7.54\left(\mathrm{~d}, J=7.7 \mathrm{~Hz}, 12 \mathrm{H}, \mathrm{H}_{\mathrm{a}}\right), 7.43\left(\mathrm{~d}, J=9.2 \mathrm{~Hz}, 6 \mathrm{H}, \mathrm{H}_{\mathrm{c}}\right), 7.28(\mathrm{~d}, J=9.2 \mathrm{~Hz}$, 6H, $\left.\mathrm{H}_{\mathrm{c}^{\prime}}\right), 7.21-6.99\left(\mathrm{~m}, 36 \mathrm{H}, \mathrm{H}_{\mathrm{b}^{\prime},{ }^{\prime}, \mathrm{h}^{\prime}, \mathrm{i}, \mathrm{i}^{\prime}}\right), 6.92\left(\mathrm{~d}, J=9.2 \mathrm{~Hz}, 6 \mathrm{H}, \mathrm{H}_{\mathrm{d}^{\prime}}\right), 2.58\left(\mathrm{~s}, 18 \mathrm{H}, \mathrm{CH}_{3}\right)$, $2.55\left(\mathrm{~s}, 18 \mathrm{H}, \mathrm{CH}_{3}\right), 2.37\left(\mathrm{~s}, 18 \mathrm{H}, \mathrm{CH}_{3}\right), 1.84\left(\mathrm{~s}, 18 \mathrm{H}, \mathrm{CH}_{3}\right), 1.61\left(\mathrm{~s}, 18 \mathrm{H}, \mathrm{CH}_{3}\right), 1.53(\mathrm{~s}$, $\left.18 \mathrm{H}, \mathrm{CH}_{3}\right)$ ppm. ${ }^{13} \mathrm{C}\left\{{ }^{1} \mathrm{H}\right\} \mathrm{NMR}\left(150 \mathrm{MHz}, \mathrm{CD}_{3} \mathrm{CN}\right) \delta=178.0$ (from $\mathrm{HMBC}, \mathrm{C}_{\mathrm{NHC}}$ ), 177.9 (from $\left.\mathrm{HMBC}, \mathrm{C}_{\mathrm{NHC}}\right), 170.6\left(\mathrm{C}_{\text {triazine }}\right), 169.4$ ( $\left.\mathrm{C}_{\text {triazine }}\right), 141.5,140.9,140.8,140.7$, $140.1,136.1,135.8,135.5,135.3,135.1,135.0,133.4,131.3,131.0,130.9,130.7,130.6$ $130.5,130.3,129.9,129.7,129.3,129.0,127.3,125.8\left(C_{e}\right), 125.6\left(C_{c}\right), 125.4\left(C_{e}\right)$, 124.1, $119.7\left(\mathrm{C}_{\mathrm{d}}\right), 119.1\left(\mathrm{C}_{\mathrm{d}^{\prime}}\right), 115.2\left(\mathrm{C}_{\mathrm{f}}\right), 115.1\left(\mathrm{C}_{\mathrm{f}^{\prime}}\right), 21.5\left(\mathrm{CH}_{3}\right), 21.4\left(\mathrm{CH}_{3}\right), 18.5$ $\left(\mathrm{CH}_{3}\right), 17.7\left(\mathrm{CH}_{3}\right), 17.3\left(\mathrm{CH}_{3}\right), 17.2\left(\mathrm{CH}_{3}\right)$ ppm. ESI-TOF MS: $m / z=1888.5994(\mathrm{calcd}$ for $\left.\left\{\left[\mathrm{Au}_{3}(\mathbf{1 b})_{2}\right]_{2}\left(\mathrm{PF}_{6}\right)_{3}\right\}^{3+} 1888.5314\right), 1380.1960$ (calcd for $\left\{\left[\mathrm{Au}_{3}(\mathbf{1 b})_{2}\right\}_{2}\left(\mathrm{PF}_{6}\right)_{2}\right]^{4+}$ 1380.1556), 1075.1534 (calcd for $\left\{\left[\mathrm{Au}_{3}(\mathbf{1 b})_{2}\right]_{2}\left(\mathrm{PF}_{6}\right)\right\}^{5+}$ 1075.1332), 871.7968 (calcd for $\left.\left\{\left[\mathrm{Au}_{3}(\mathbf{1 b})_{2}\right]_{2}\right\}^{6+} 871.7875\right)$.

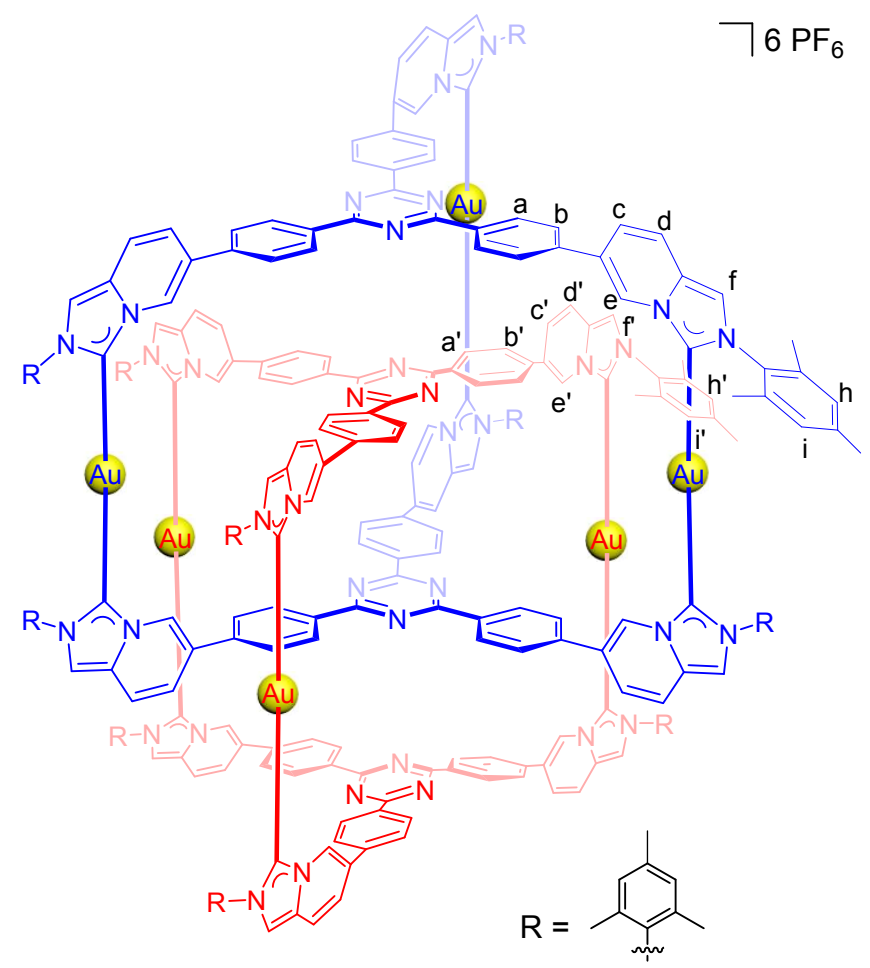




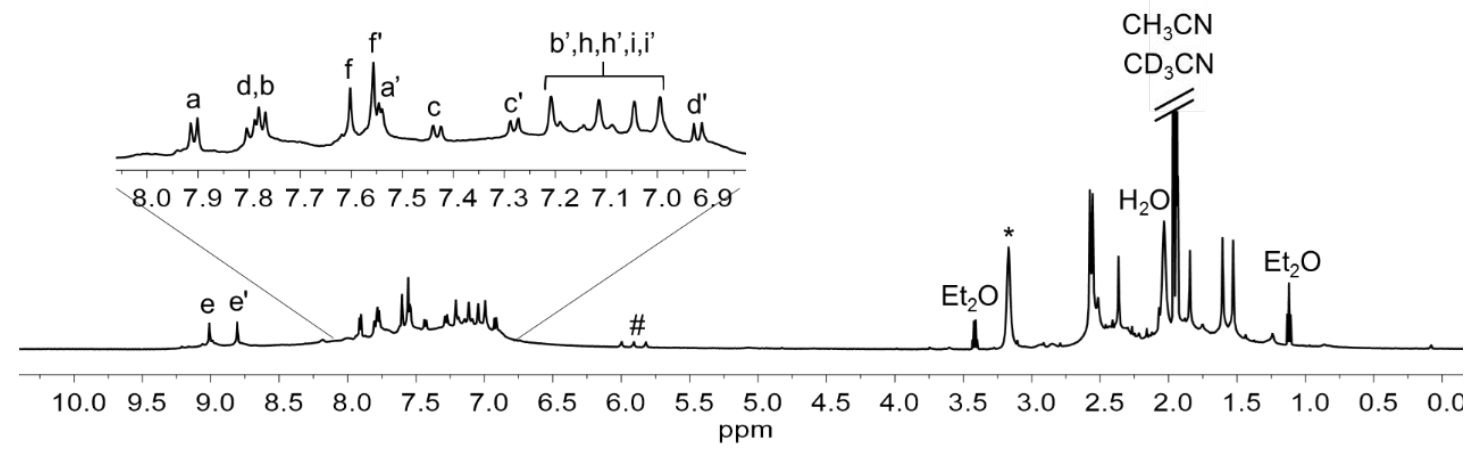

Figure S39. ${ }^{1} \mathrm{H}$ NMR spectrum of $\left[\mathrm{Au}_{3}(\mathbf{1 b})_{2}\right]_{2}\left(\mathrm{PF}_{6}\right)_{6}\left(600 \mathrm{MHz}, \mathrm{CD}_{3} \mathrm{CN} ; *\right.$, \# = residue THT and inorganic salt).

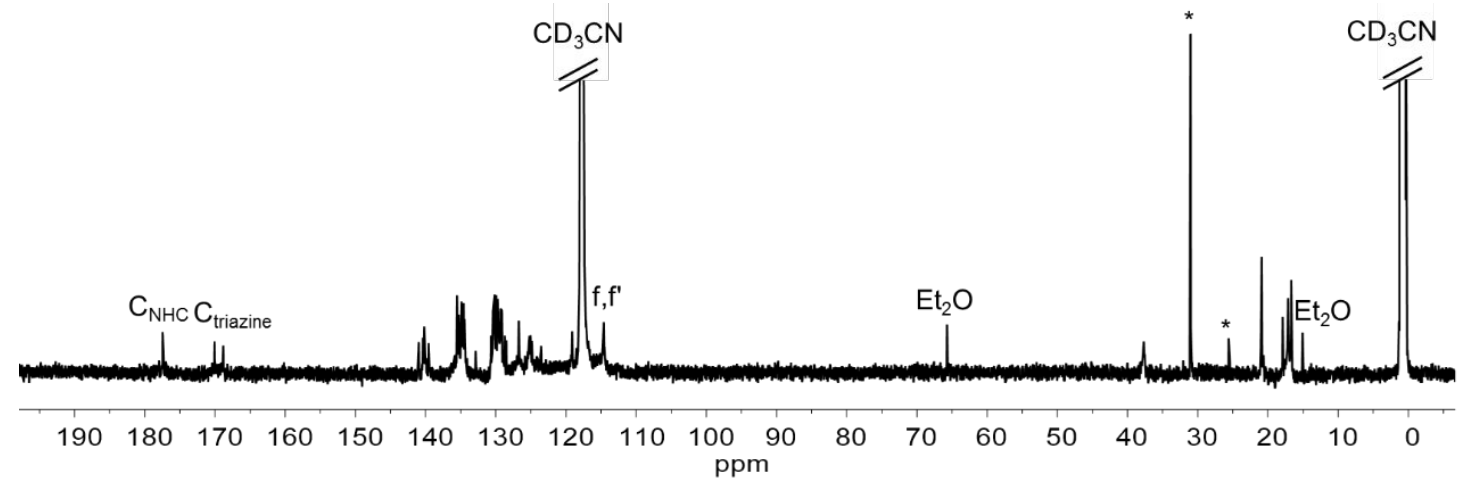

Figure S40. ${ }^{13} \mathrm{C}\left\{{ }^{1} \mathrm{H}\right\}$ NMR spectrum of $\left[\mathrm{Au}_{3}(\mathbf{1 b})_{2}\right]_{2}\left(\mathrm{PF}_{6}\right)_{6}\left(150 \mathrm{MHz}, \mathrm{CD}_{3} \mathrm{CN}\right.$; * = residue THT). 


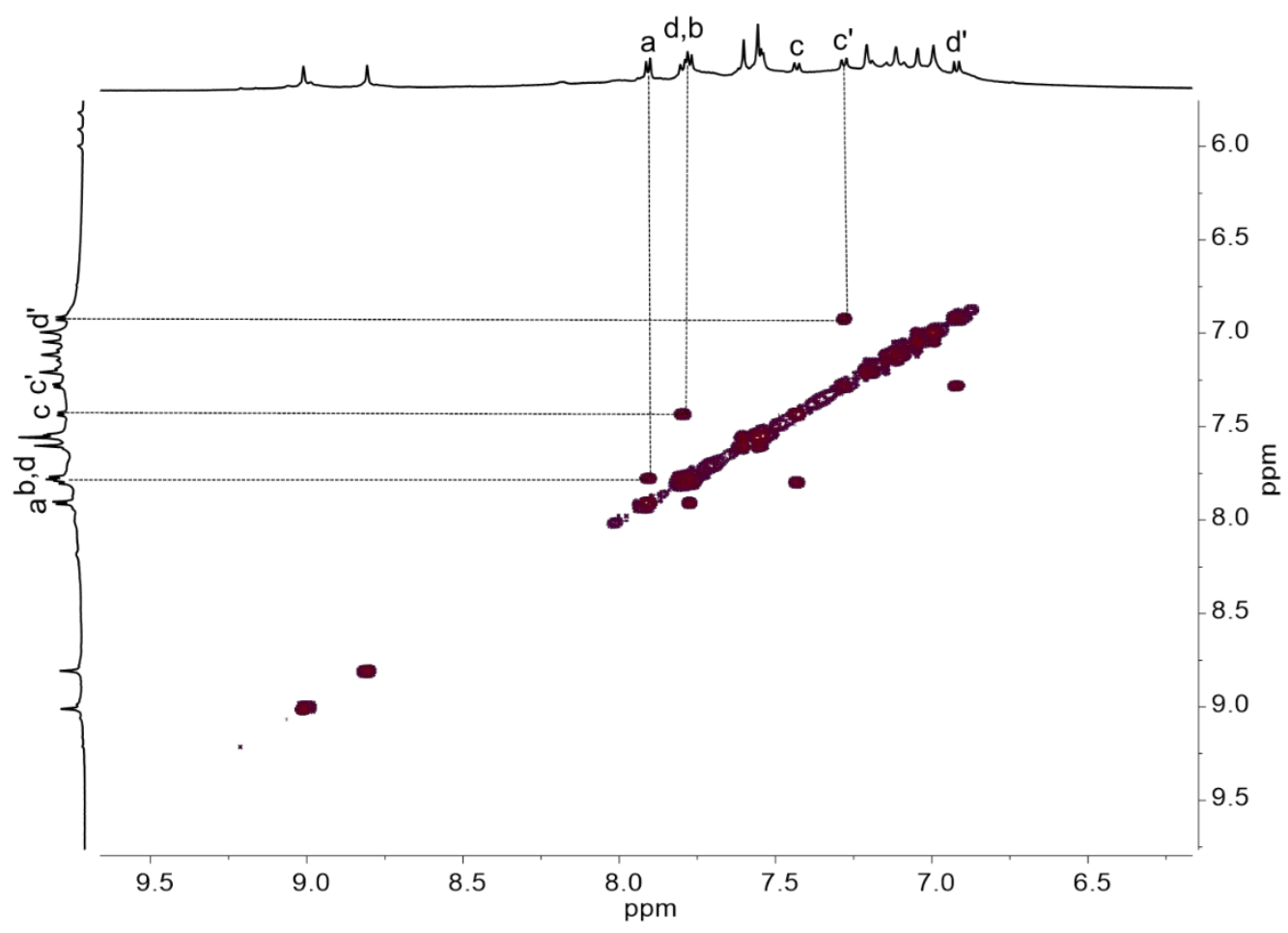

Figure S41. ${ }^{1} \mathrm{H}-{ }^{1} \mathrm{H}$ COSY spectrum $\left(600 \mathrm{MHz}, \mathrm{CD}_{3} \mathrm{CN}\right)$ of $\left[\mathrm{Au}_{3}(\mathbf{1 b})_{2}\right]_{2}\left(\mathrm{PF}_{6}\right)_{6}$.

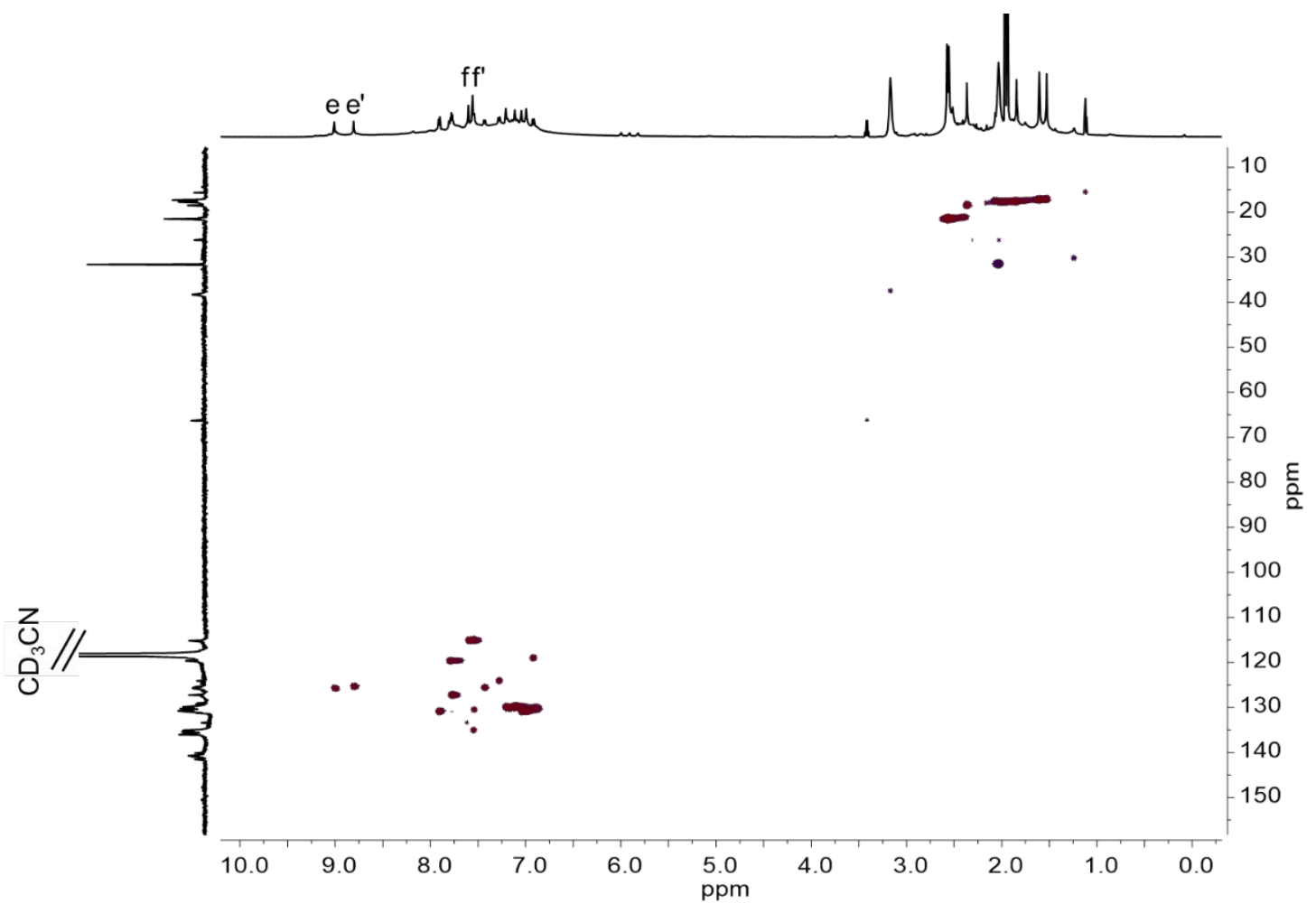

Figure $\mathbf{S 4 2 .}{ }^{1} \mathrm{H}-{ }^{13} \mathrm{C}$ HSQC spectrum $\left(600 \mathrm{MHz}, \mathrm{CD}_{3} \mathrm{CN}\right)$ of $\left[\mathrm{Au}_{3}(\mathbf{1 b})_{2}\right]_{2}\left(\mathrm{PF}_{6}\right)_{6}$. 


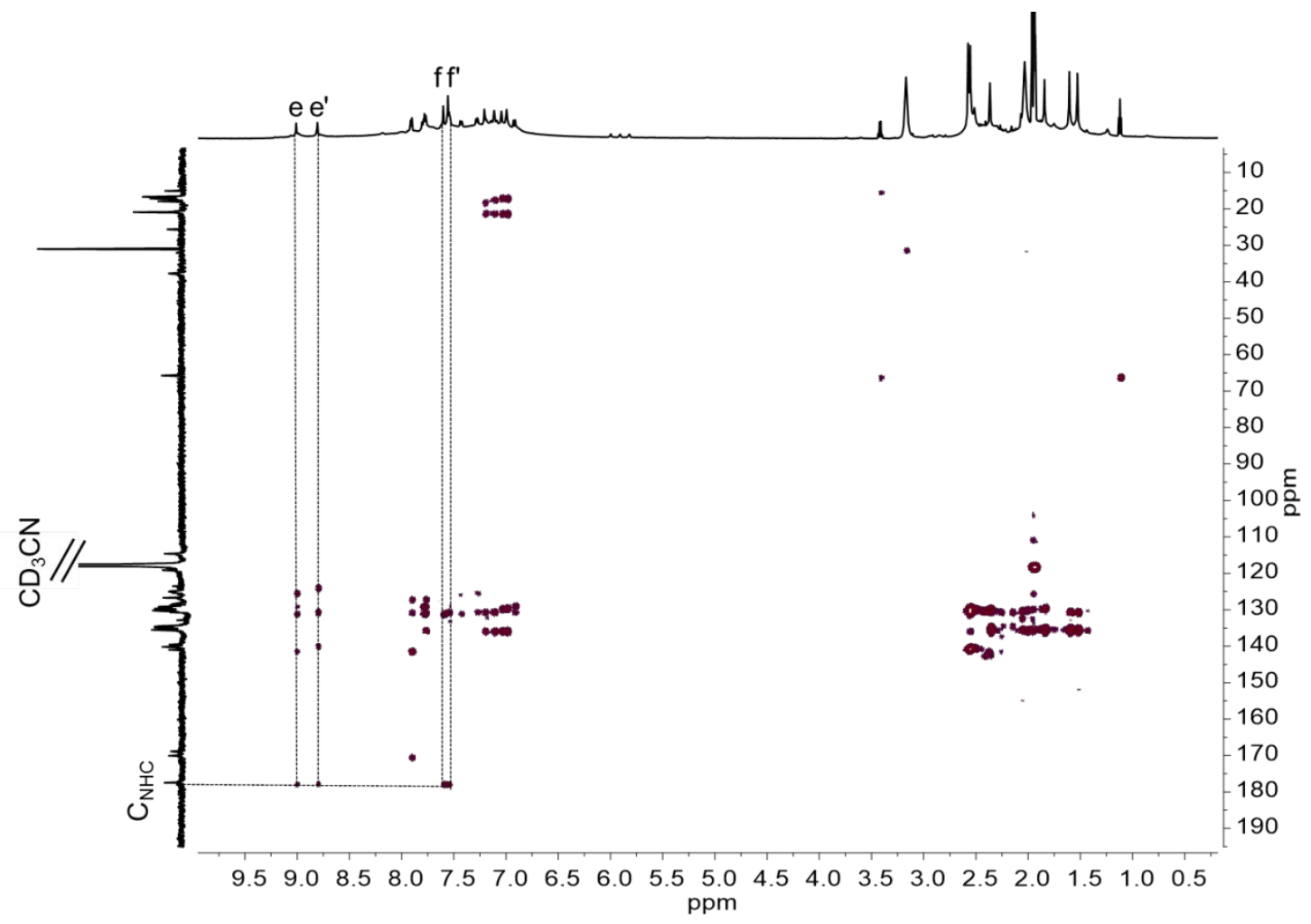

Figure S43. ${ }^{1} \mathrm{H}-{ }^{13} \mathrm{C}$ HMBC spectrum $\left(600 \mathrm{MHz}, \mathrm{CD}_{3} \mathrm{CN}\right)$ of $\left[\mathrm{Au}_{3}(\mathbf{1 b})_{2}\right]_{2}\left(\mathrm{PF}_{6}\right)_{6}$.

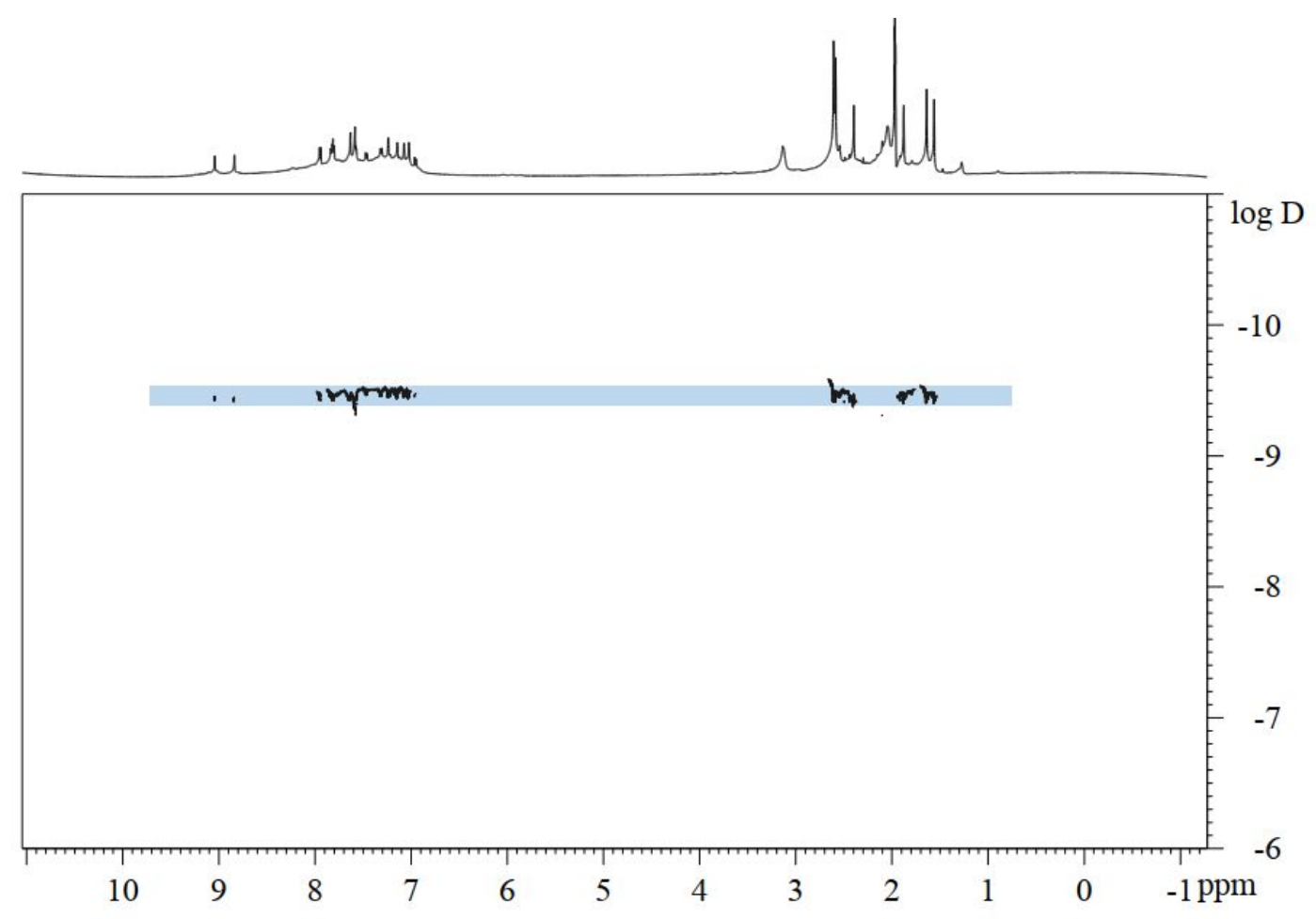

Figure S44. ${ }^{1} \mathrm{H}$ DOSY spectrum $\left(600 \mathrm{MHz}, \mathrm{CD}_{3} \mathrm{CN}\right)$ of $\left[\mathrm{Au}_{3}(\mathbf{1 b})_{2}\right]_{2}\left(\mathrm{PF}_{6}\right)_{6} \cdot[\mathrm{D}=3.498$

$\left.\times 10^{-10} \mathrm{~m}^{2} \mathrm{~s}^{-1}(\log \mathrm{D}=-9.46)\right]$. 


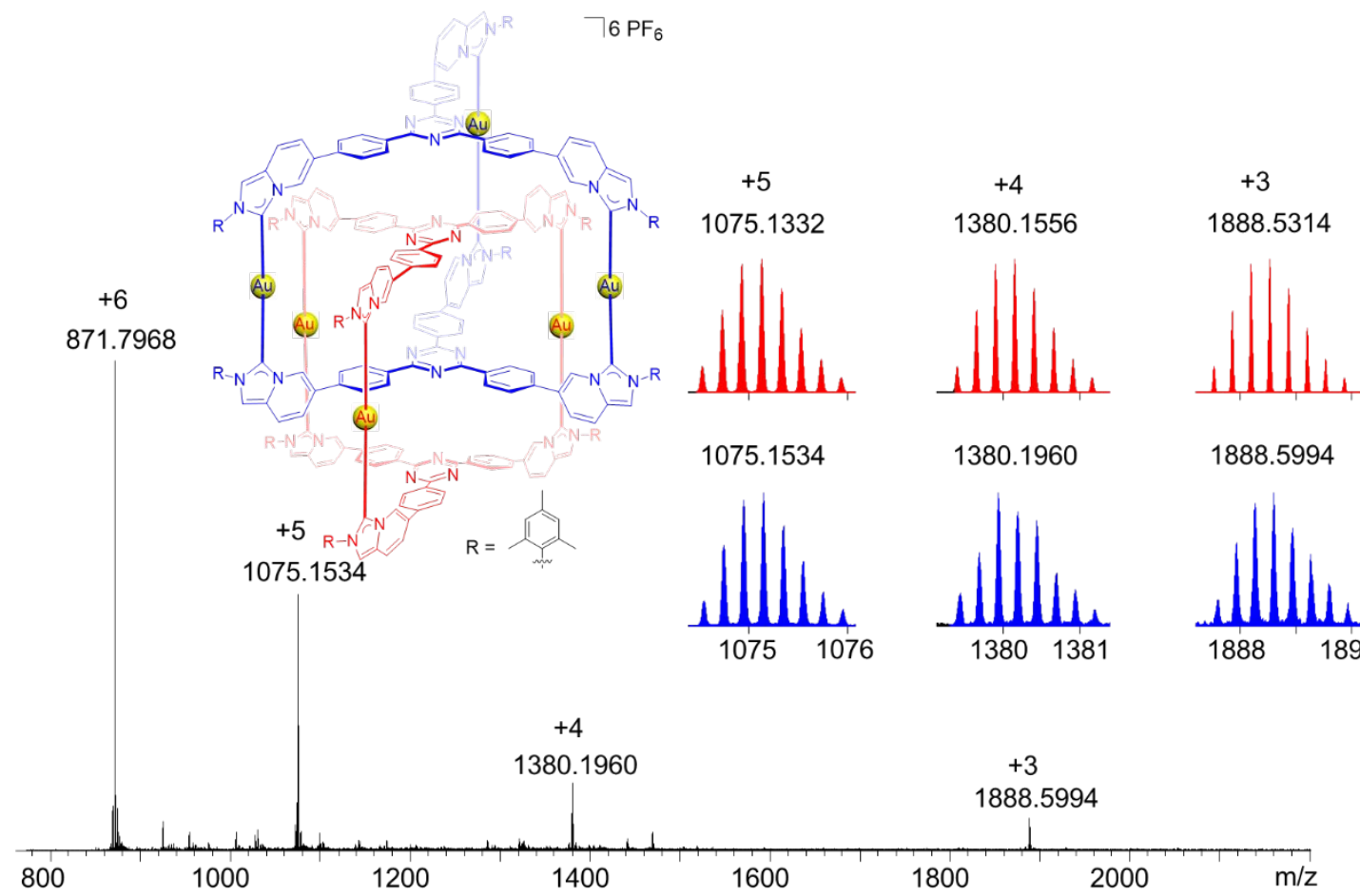

Figure S45. ESI-TOF mass spectrum of $\left[\mathrm{Au}_{3}(\mathbf{1 b})_{2}\right]_{2}\left(\mathrm{PF}_{6}\right)_{6}$ with isotope distribution for selected peaks (experimental in blue, calculated in red, $\mathrm{PF}_{6}^{-}$counterions).

\subsection{Synthesis of complex $\left[\mathrm{Au}_{3}(1 \mathrm{c})_{2}\right]_{2}\left(\mathrm{PF}_{6}\right)_{6}$}
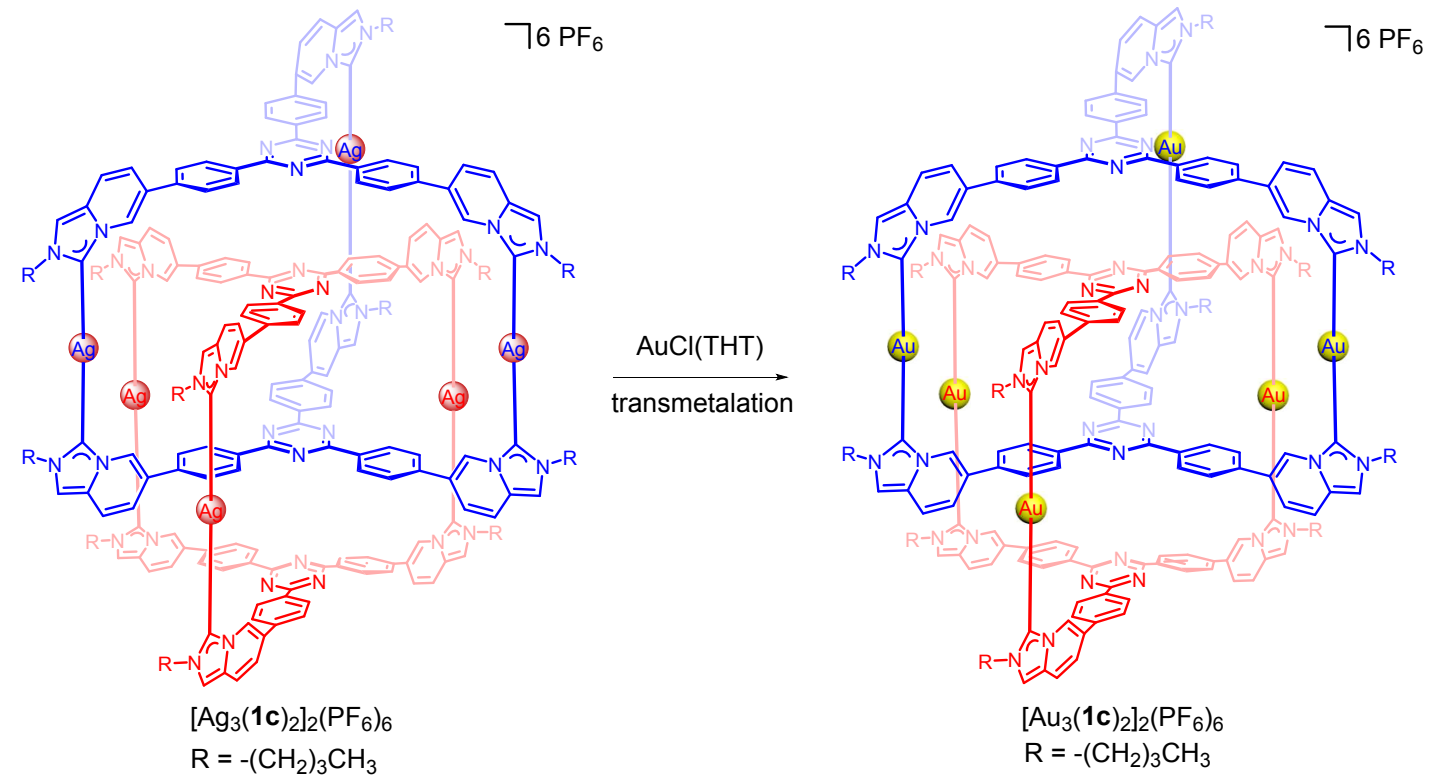

Scheme S6. Synthesis of Complex $\left[\mathrm{Au}_{3}(\mathbf{1 c})_{2}\right]_{2}\left(\mathrm{PF}_{6}\right)_{6}$.

Complex $\left[\mathrm{Au}_{3}(\mathbf{1 c})_{2}\right]_{2}\left(\mathrm{PF}_{6}\right)_{6}$ was prepared from $\left[\mathrm{Ag}_{3}(\mathbf{1 c})_{2}\right]_{2}\left(\mathrm{PF}_{6}\right)_{6}(30 \mathrm{mg}, 0.006 \mathrm{mmol})$ 
and $\mathrm{AuCl}(\mathrm{THT})(12 \mathrm{mg}, 0.037 \mathrm{mmol})$. Yield: $27 \mathrm{mg}$ (0.005 mmol, 83\%). ${ }^{1} \mathrm{H}$ NMR (600 $\left.\mathrm{MHz}, \mathrm{CD}_{3} \mathrm{CN}\right) \delta=8.79\left(\mathrm{~s}, 6 \mathrm{H}, \mathrm{H}_{\mathrm{e}}\right), 8.35\left(\mathrm{~s}, 6 \mathrm{H}, \mathrm{H}_{\mathrm{e}}\right), 8.14\left(\mathrm{br} \mathrm{s}, 12 \mathrm{H}, \mathrm{H}_{\mathrm{a}}\right), 7.60\left(\mathrm{~s}, \mathrm{H}_{\mathrm{f}}\right)$, $7.55\left(\mathrm{~d}, J=9.2 \mathrm{~Hz}, \mathrm{H}_{\mathrm{d}}\right), 7.46\left(\mathrm{~s}, \mathrm{H}_{\mathrm{f}^{\prime}}\right), 7.25\left(\mathrm{~m}, \mathrm{H}_{\mathrm{a}^{\prime}, \mathrm{b}, \mathrm{b}^{\prime}}\right), 7.19\left(\mathrm{~d}, J=9.2 \mathrm{~Hz}, \mathrm{H}_{\mathrm{d}^{\prime}}\right), 7.09$ $\left(\mathrm{d}, J=9.2 \mathrm{~Hz}, \mathrm{H}_{\mathrm{c}}\right), 6.83\left(\mathrm{~d}, J=9.2 \mathrm{~Hz}, \mathrm{H}_{\mathrm{c}^{\prime}}\right), 4.81-4.77\left(\mathrm{~m}, 6 \mathrm{H}, \mathrm{H}_{\mathrm{h} / \mathrm{h}^{\prime}}\right), 4.62-4.57(\mathrm{~m}$, $\left.6 \mathrm{H}, \mathrm{H}_{\mathrm{h} / \mathrm{h}^{\prime}}\right), 4.42-4.37\left(\mathrm{~m}, 6 \mathrm{H}, \mathrm{H}_{\mathrm{h} / \mathrm{h}}\right), 4.21-4.16\left(\mathrm{~m}, 6 \mathrm{H}, \mathrm{H}_{\mathrm{h} / \mathrm{h}}\right), 2.05-1.99\left(\mathrm{~m}, 24 \mathrm{H}, \mathrm{H}_{\mathrm{i}, \mathrm{i}^{\prime}}\right)$, 1.51-1.38 (m, 24H, $\left.\mathrm{H}_{\mathrm{j}, \mathrm{j}^{\mathrm{j}}}\right), 1.06-1.00\left(\mathrm{~m}, 36 \mathrm{H}, \mathrm{H}_{\mathrm{k}, \mathrm{k}^{\prime}}\right)$ ppm. ESI-TOF MS: $m / z=$ 1193.8618 (calcd for $\left\{\left[\mathrm{Au}_{3}(\mathbf{1} \mathbf{c})_{2}\right]_{2}\left(\mathrm{PF}_{6}\right)_{2}\right\}^{4+} 1193.8602$ ), 926.0953 (calcd for $\left\{\left[\mathrm{Au}_{3}(\mathbf{1 c})_{2}\right]_{2}\left(\mathrm{PF}_{6}\right)\right\}^{5+}$ 926.0953), $747.5853\left(\right.$ calcd for $\left.\left\{\left[\mathrm{Au}_{3}(\mathbf{1 c})_{2}\right]_{2}\right\}^{6+} 747.5854\right)$.
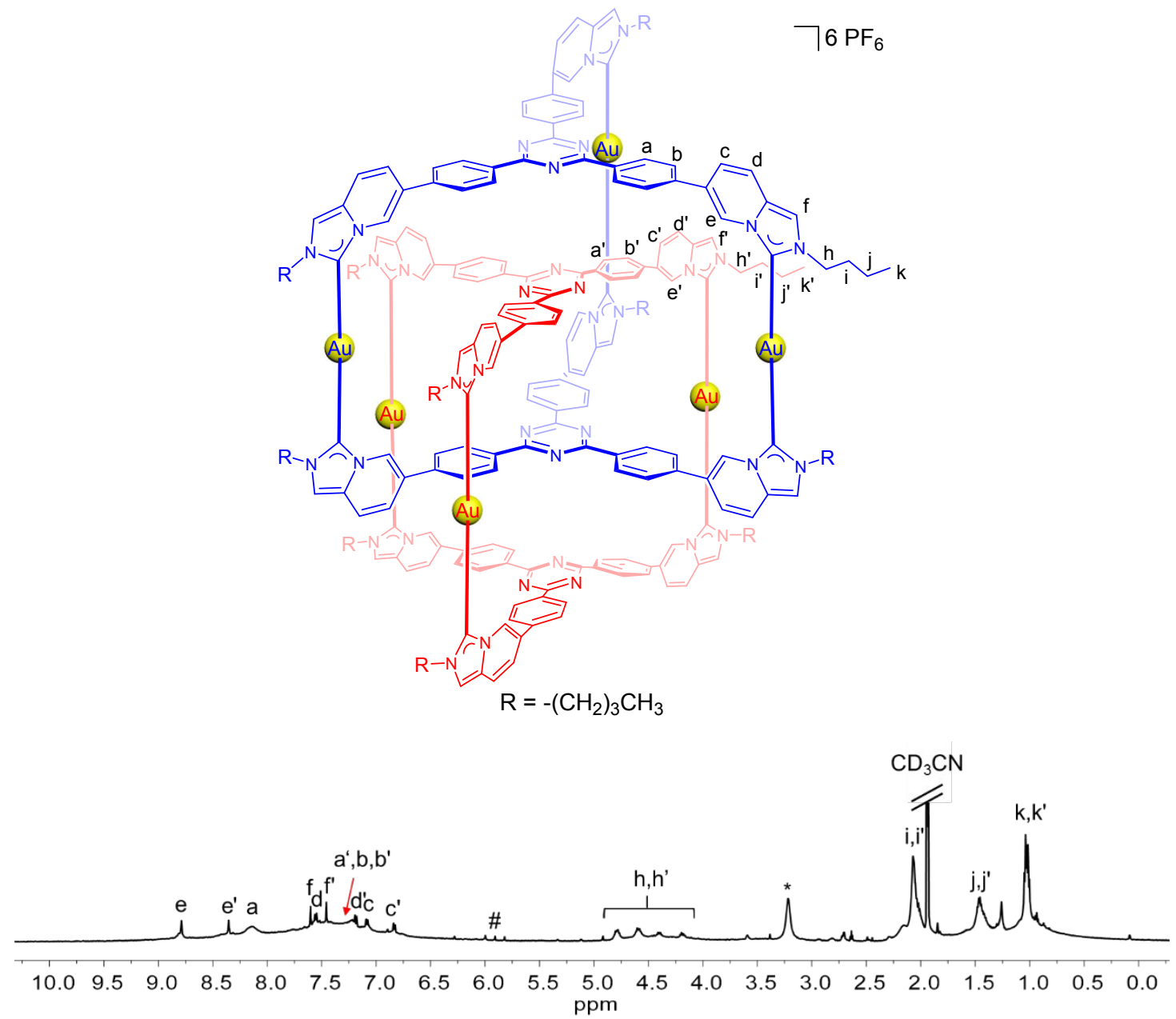

Figure S46. ${ }^{1} \mathrm{H} \mathrm{NMR}$ spectrum of $\left[\mathrm{Au}_{3}(\mathbf{1} \mathbf{c})_{2}\right]_{2}\left(\mathrm{PF}_{6}\right)_{6}\left(600 \mathrm{MHz}, \mathrm{CD}_{3} \mathrm{CN}\right.$; *, \# = residue THT and inorganic salt). 


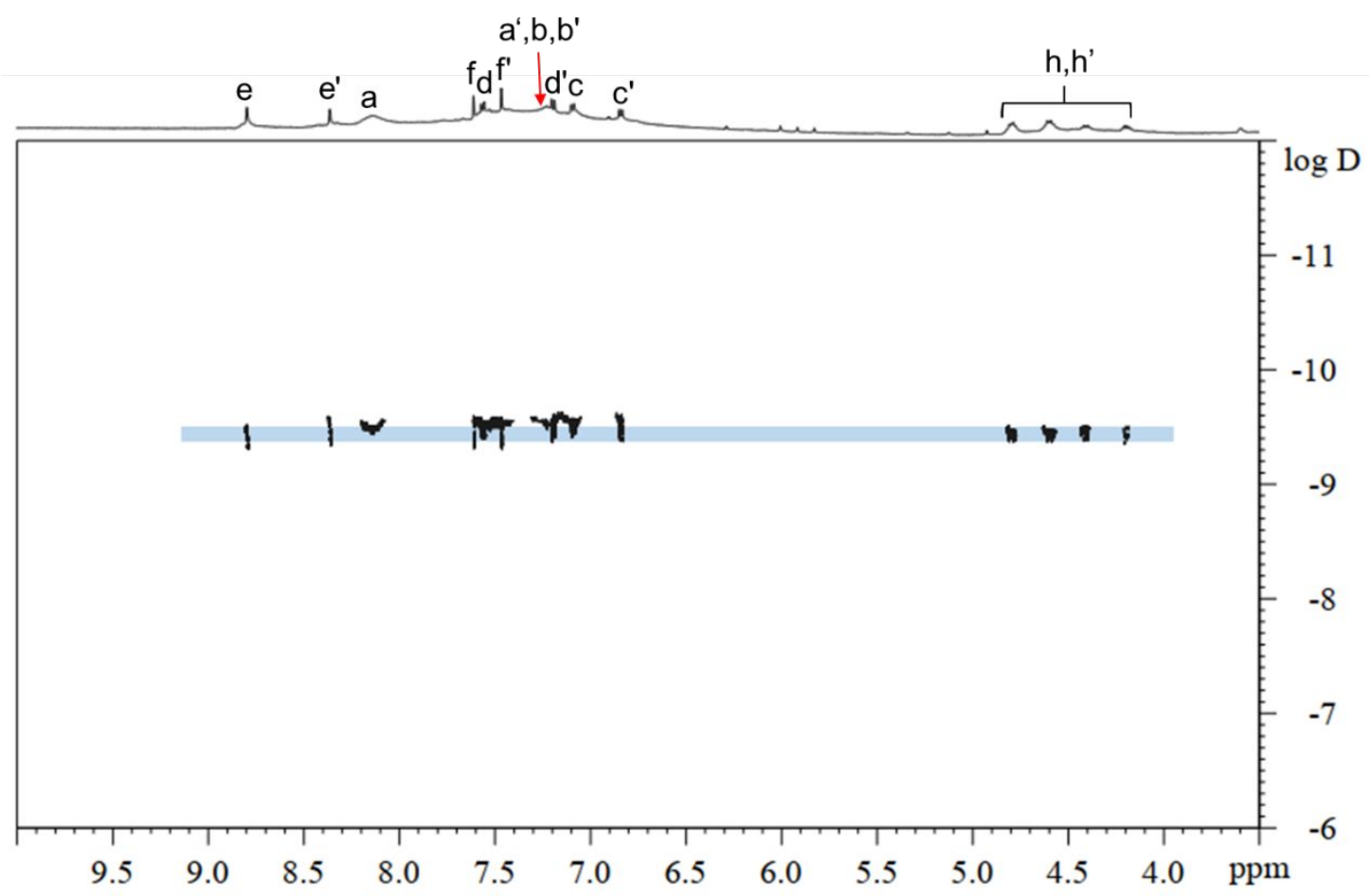

Figure S47. ${ }^{1} \mathrm{H}$ DOSY spectrum of $\left[\mathrm{Au}_{3}(\mathbf{1} \mathrm{c})_{2}\right]_{2}\left(\mathrm{PF}_{6}\right)_{6}\left(600 \mathrm{MHz}, \mathrm{CD}_{3} \mathrm{CN}\right) .[\mathrm{D}=3.576$ $\left.\times 10^{-10} \mathrm{~m}^{2} \mathrm{~s}^{-1}(\log \mathrm{D}=-9.45)\right]$.

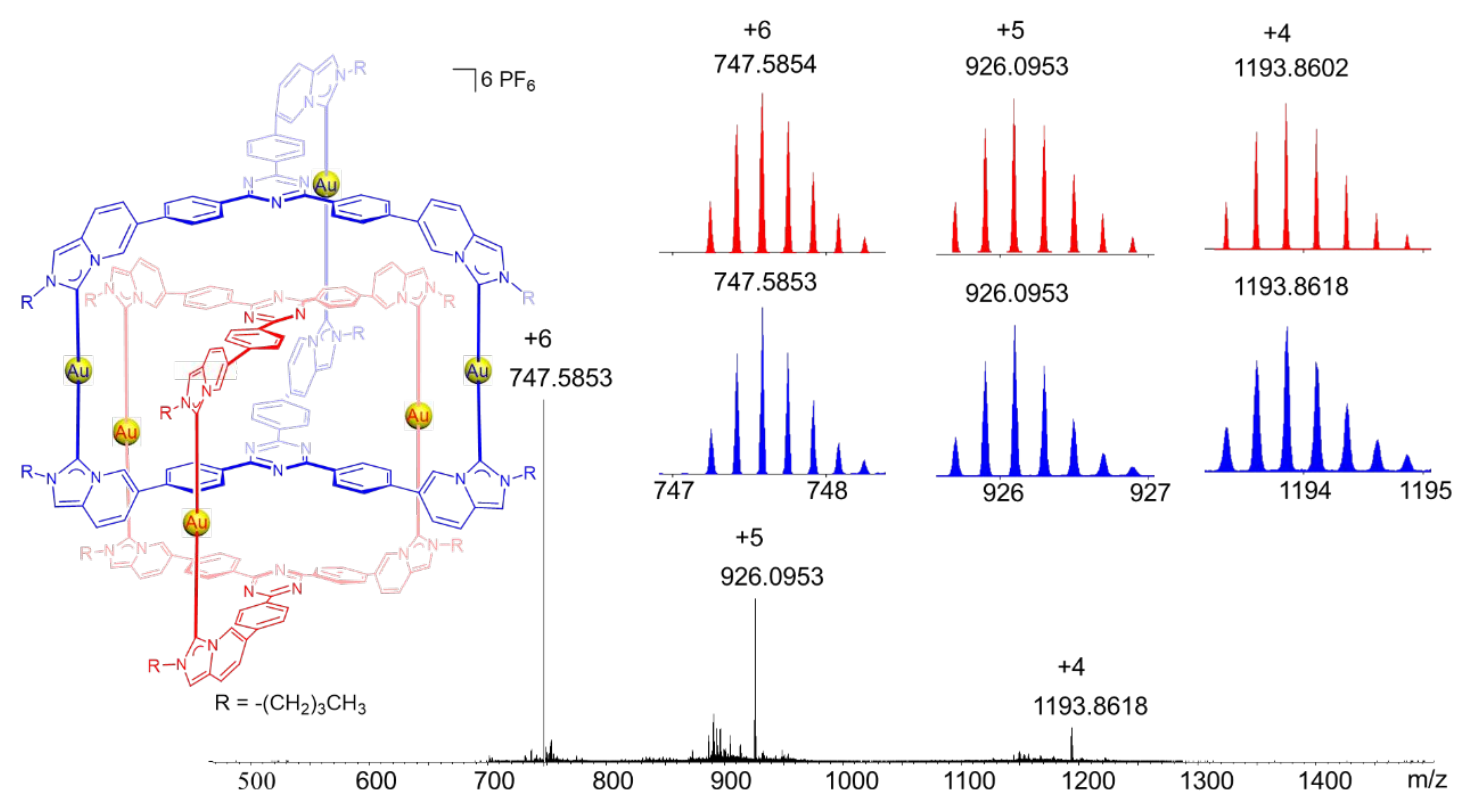

Figure S48. ESI-TOF mass spectrum of $\left[\mathrm{Au}_{3}(\mathbf{1 c})_{2}\right]_{2}\left(\mathrm{PF}_{6}\right)_{6}$ with isotope distribution for selected peaks (experimental in blue, calculated in red, $\mathrm{PF}_{6}{ }^{-}$counterions). 
5. UV-vis absorption and emission spectra of $\mathrm{H}_{3}-\mathrm{L}\left(\mathrm{PF}_{6}\right)_{3},\left[\mathrm{Ag}_{3}(\mathrm{~L})_{2}\right]_{2}\left(\mathrm{PF}_{6}\right)_{6}(\mathrm{~L}=$ $1 a-1 c)$ and $\left[\mathrm{Au}_{3}(\mathrm{~L})_{2}\right]_{2}\left(\mathrm{PF}_{6}\right)_{6}(\mathrm{~L}=1 \mathrm{~b}, 1 \mathrm{c})$.

(a)

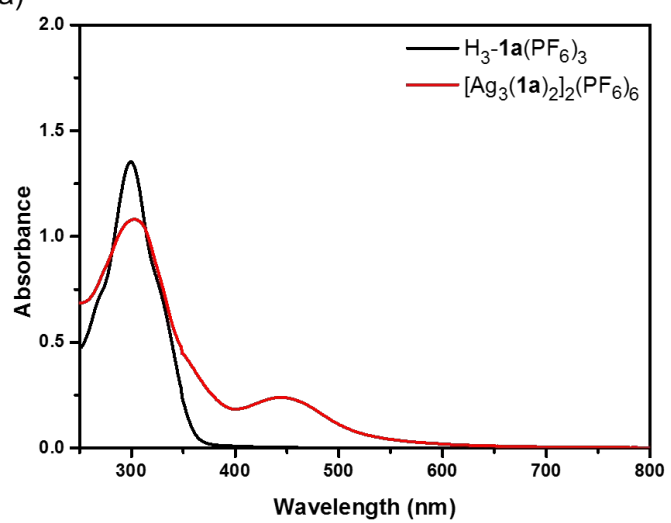

(b)

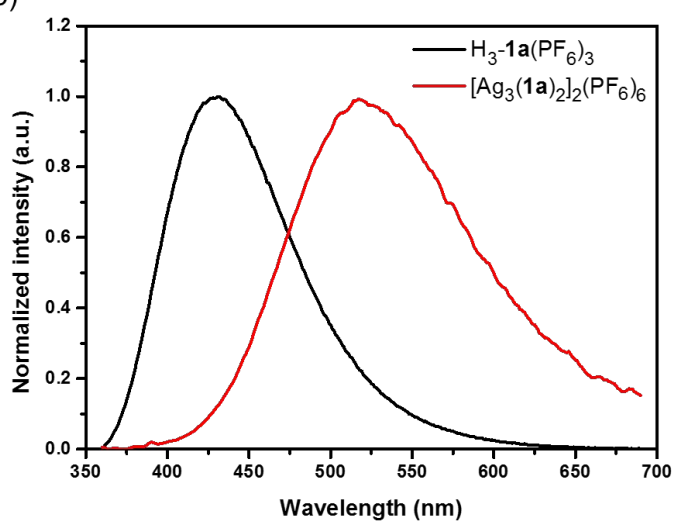

Figure S49. (a) UV-vis absorption spectra $\left(\mathrm{CH}_{3} \mathrm{CN}, c=1 \times 10^{-5} \mathrm{M}\right)$, (b) normalized emission spectra of $\mathrm{H}_{3}-\mathbf{1 a}\left(\mathrm{PF}_{6}\right)_{3}$ and $\left[\mathrm{Ag}_{3}\left(\mathbf{1 a}_{2}\right]_{2}\left(\mathrm{PF}_{6}\right)_{6}\left(\mathrm{CH}_{3} \mathrm{CN}, \lambda_{\mathrm{ex}}=350 \mathrm{~nm}, c=1 \mathrm{x}\right.\right.$ $\left.10^{-5} \mathrm{M}\right)$.

(a)

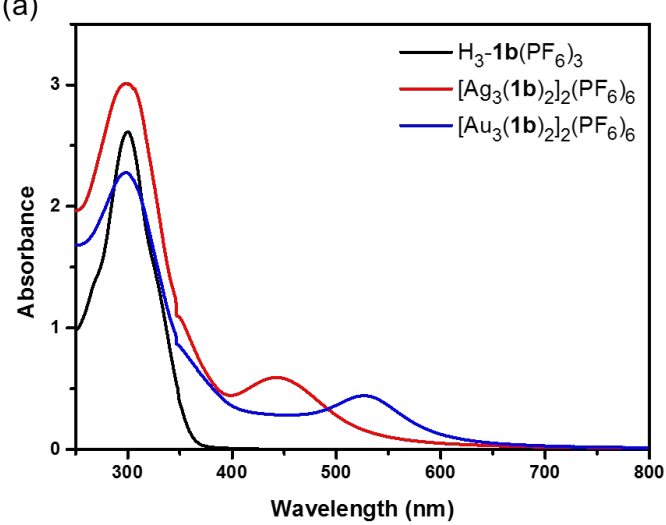

(b)

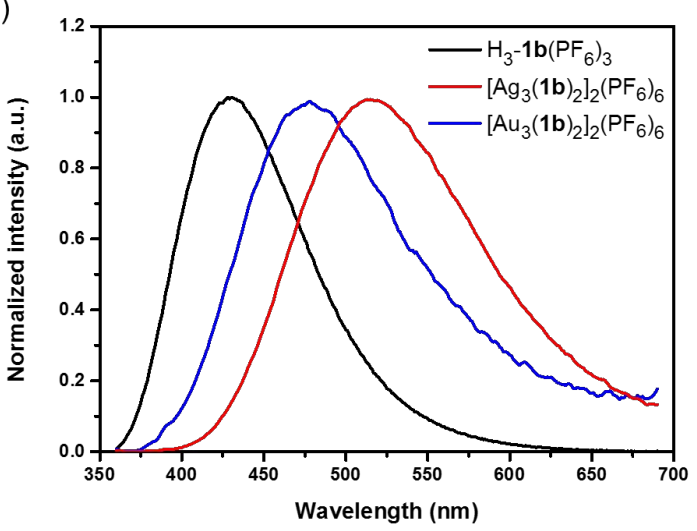

Figure S50. (a) UV-vis absorption spectra $\left(\mathrm{CH}_{3} \mathrm{CN}, c=1 \times 10^{-5} \mathrm{M}\right)$, (b) normalized emission spectra of $\mathrm{H}_{3}-\mathbf{1 b}\left(\mathrm{PF}_{6}\right)_{3},\left[\mathrm{Ag}_{3}(\mathbf{1 b})_{2}\right]_{2}\left(\mathrm{PF}_{6}\right)_{6}$ and $\left[\mathrm{Au}_{3}(\mathbf{1 b})_{2}\right]_{2}\left(\mathrm{PF}_{6}\right)_{6}\left(\mathrm{CH}_{3} \mathrm{CN}, \lambda_{\mathrm{ex}}\right.$ $\left.=350 \mathrm{~nm}, c=1 \times 10^{-5} \mathrm{M}\right)$. 
(a)

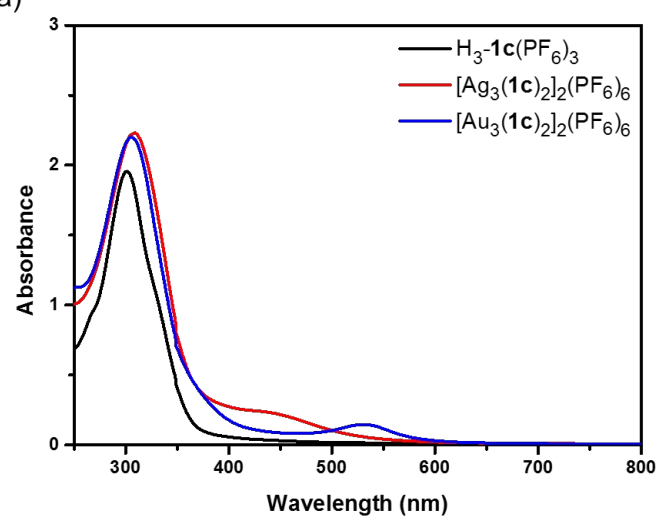

(b)

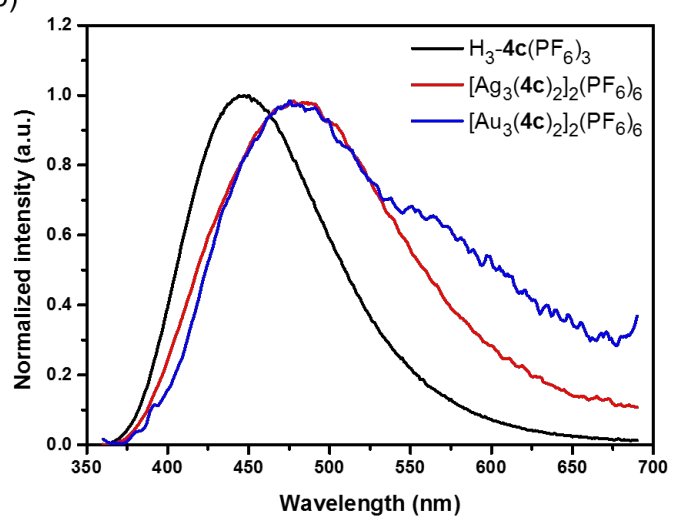

Figure S51. (a) UV-vis absorption spectra $\left(\mathrm{CH}_{3} \mathrm{CN}, c=1 \times 10^{-5} \mathrm{M}\right)$, (b) normalized emission spectra of $\mathrm{H}_{3}-\mathbf{1} \mathbf{c}\left(\mathrm{PF}_{6}\right)_{3},\left[\mathrm{Ag}_{3}(\mathbf{1} \mathbf{c})_{2}\right]_{2}\left(\mathrm{PF}_{6}\right)_{6}$ and $\left[\mathrm{Au}_{3}(\mathbf{1} \mathbf{c})_{2}\right]_{2}\left(\mathrm{PF}_{6}\right)_{6}\left(\mathrm{CH}_{3} \mathrm{CN}, \lambda_{\mathrm{ex}}\right.$ $\left.=350 \mathrm{~nm}, c=1 \times 10^{-5} \mathrm{M}\right)$.

6. Stability of Triply Interlocked $\left[\mathrm{M}_{3}(\mathrm{~L})_{2}\right]_{2}(\mathrm{M}=\mathrm{Ag}$, Au) Cages.

(a) Le Nollumbura

(b) dullumkn III

(c) Cllounh,

(d) Munt

Figure S52. ${ }^{1} \mathrm{H}$ NMR spectra $\left(600 \mathrm{MHz}, \mathrm{CD}_{3} \mathrm{CN}\right)$ of $\left[\mathrm{Ag}_{3}(\mathbf{1 a})_{2}\right]_{2}\left(\mathrm{PF}_{6}\right)_{6}$ at different temperature, a) $313 \mathrm{~K}$, b) $293 \mathrm{~K}$, c) $273 \mathrm{~K}$, and d) $253 \mathrm{~K}$. 


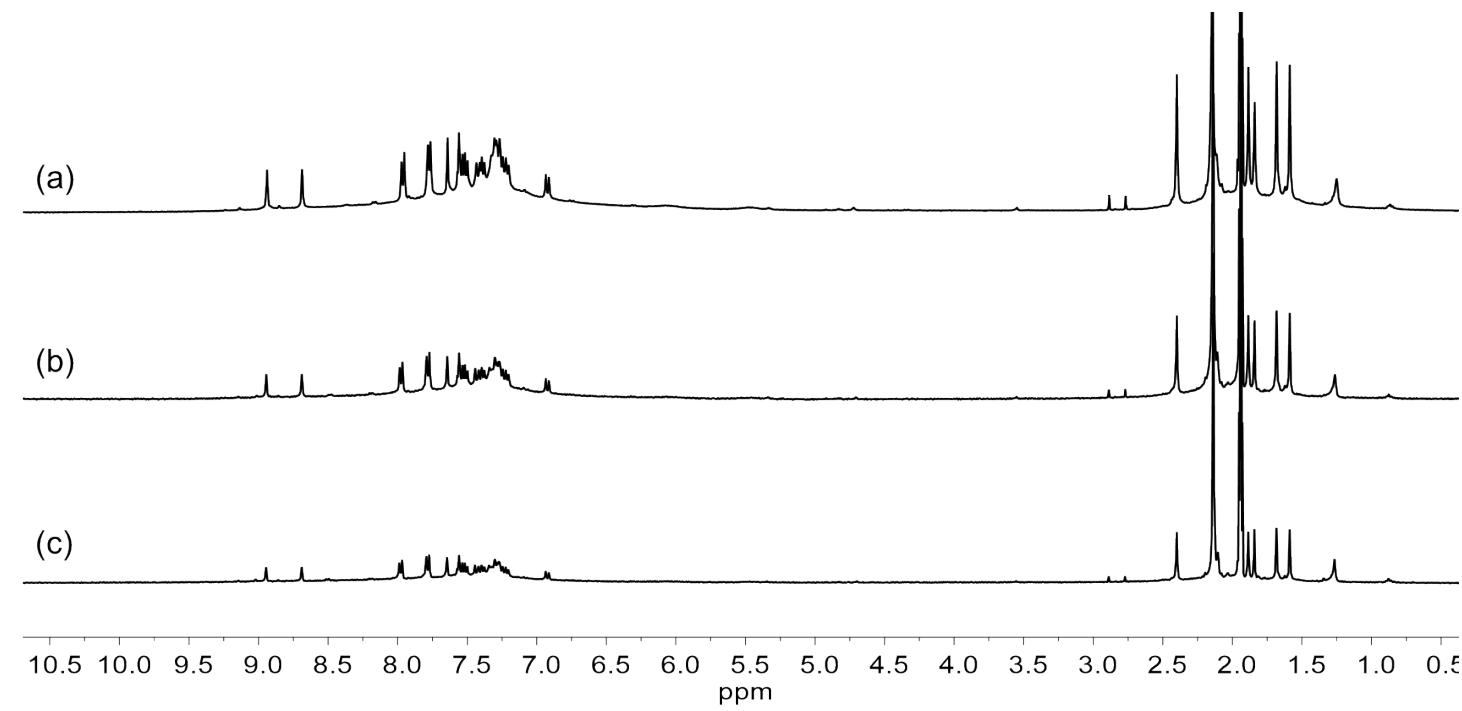

Figure S53. ${ }^{1} \mathrm{H}$ NMR spectra $\left(600 \mathrm{MHz}, \mathrm{CD}_{3} \mathrm{CN}\right)$ of $\left[\mathrm{Ag}_{3}(\mathbf{1 a})_{2}\right]_{2}\left(\mathrm{PF}_{6}\right)_{6}$ at different concentrations. a) $5.5 \mathrm{mmol} / \mathrm{L}$ (nearly saturated solution), b) $3.3 \mathrm{mmol} / \mathrm{L}$, c) 1.1 $\mathrm{mmol} / \mathrm{L}$.

(a) Uhendure

(b) Moldudure

(c) Mllowlde

(d) Mundulum

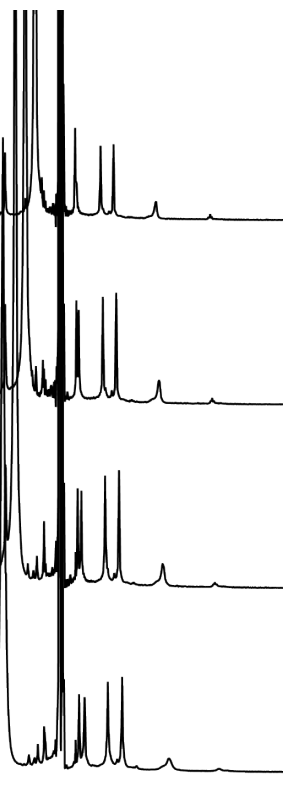

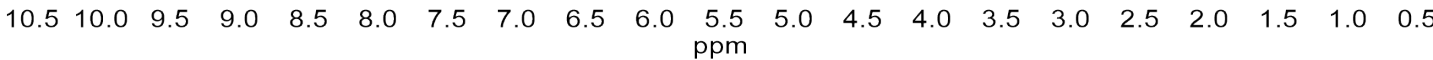

Figure S54. ${ }^{1} \mathrm{H}$ NMR spectra $\left(600 \mathrm{MHz}, \mathrm{CD}_{3} \mathrm{CN}\right)$ of $\left[\mathrm{Ag}_{3}(\mathbf{1 b})_{2}\right]_{2}\left(\mathrm{PF}_{6}\right)_{6}$ at different temperature, a) $313 \mathrm{~K}$, b) $293 \mathrm{~K}$, c) $273 \mathrm{~K}$, and d) $253 \mathrm{~K}$. 
(a) Murdun

(b)

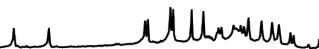

(c) Whllit.

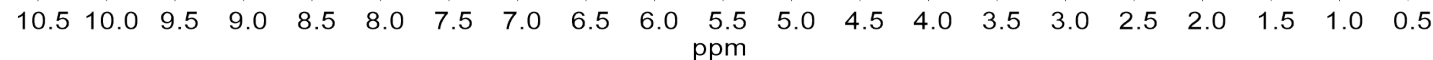

Figure S55. ${ }^{1} \mathrm{H}$ NMR spectra $\left(600 \mathrm{MHz}, \mathrm{CD}_{3} \mathrm{CN}\right)$ of $\left[\mathrm{Ag}_{3}(\mathbf{1 b})_{2}\right]_{2}\left(\mathrm{PF}_{6}\right)_{6}$ at different concentrations. a) $5.5 \mathrm{mmol} / \mathrm{L}$ (nearly saturated solution), b) $3.3 \mathrm{mmol} / \mathrm{L}$, c) 1.1 $\mathrm{mmol} / \mathrm{L}$.

(a) Lheloluen

(b) aln

(c)

(d)

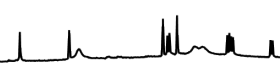
han rul

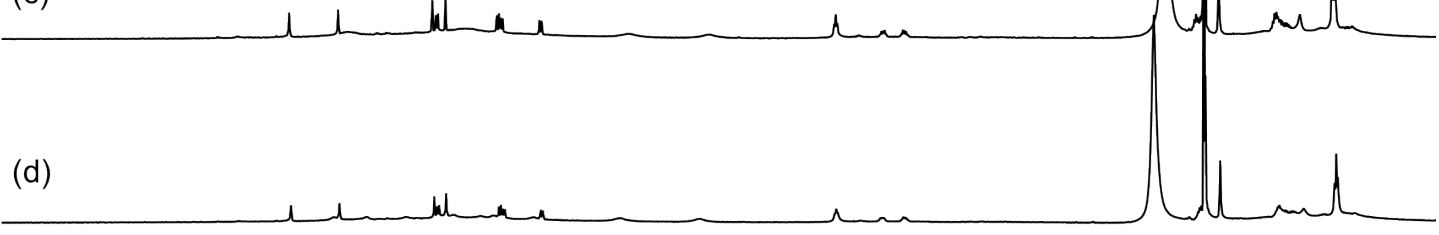

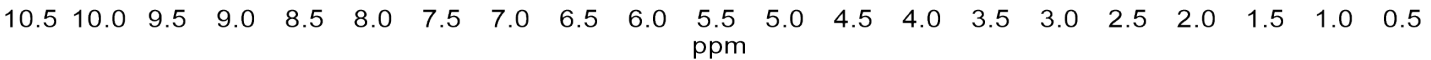

Figure S56. ${ }^{1} \mathrm{H}$ NMR spectra $\left(600 \mathrm{MHz}, \mathrm{CD}_{3} \mathrm{CN}\right)$ of $\left[\mathrm{Ag}_{3}(\mathbf{1 c})_{2}\right]_{2}\left(\mathrm{PF}_{6}\right)_{6}$ at different temperature, a) $313 \mathrm{~K}$, b) $293 \mathrm{~K}$, c) $273 \mathrm{~K}$, and d) $253 \mathrm{~K}$. 


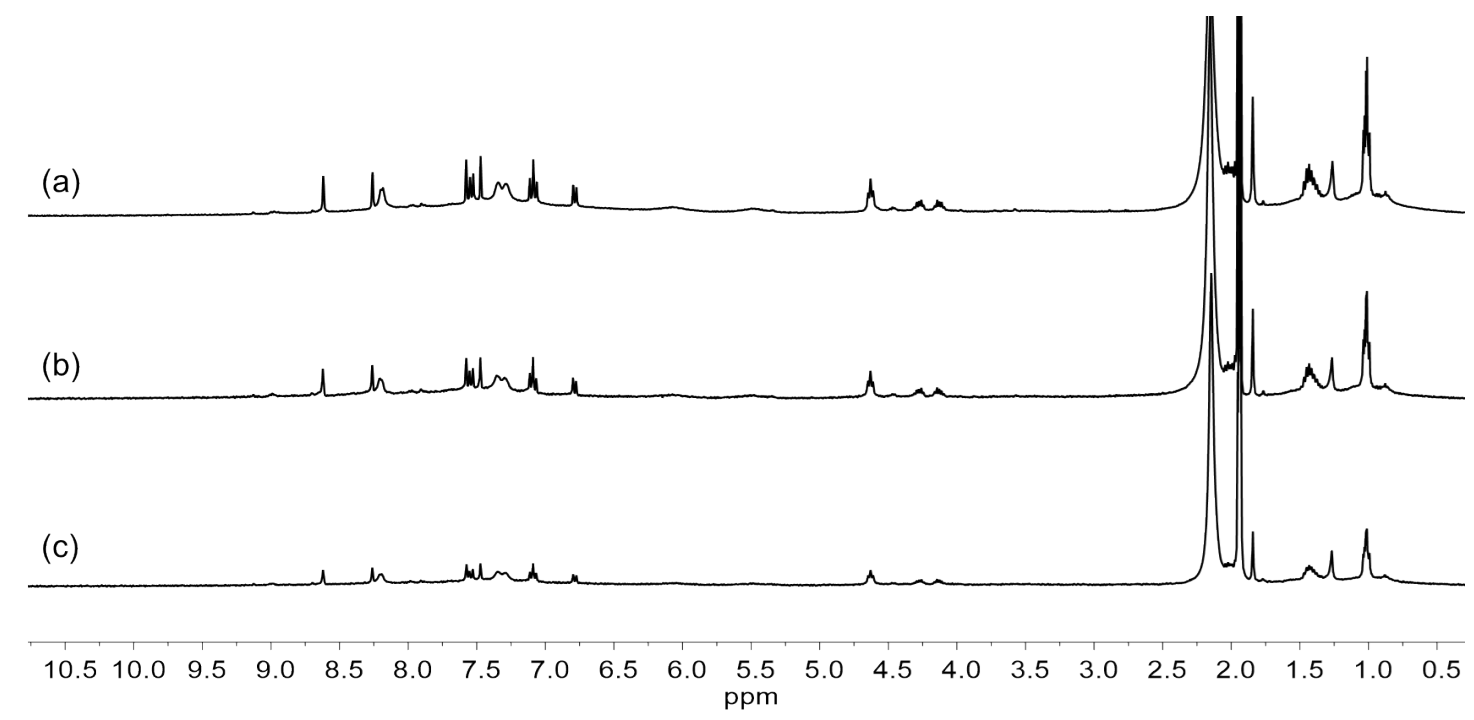

Figure S57. ${ }^{1} \mathrm{H}$ NMR spectra $\left(600 \mathrm{MHz}, \mathrm{CD}_{3} \mathrm{CN}\right)$ of $\left[\mathrm{Ag}_{3}(\mathbf{1 c})_{2}\right]_{2}\left(\mathrm{PF}_{6}\right)_{6}$ at different concentrations. a) $5.5 \mathrm{mmol} / \mathrm{L}$ (nearly saturated solution), b) $3.3 \mathrm{mmol} / \mathrm{L}$, c) 1.1 $\mathrm{mmol} / \mathrm{L}$.

(a)

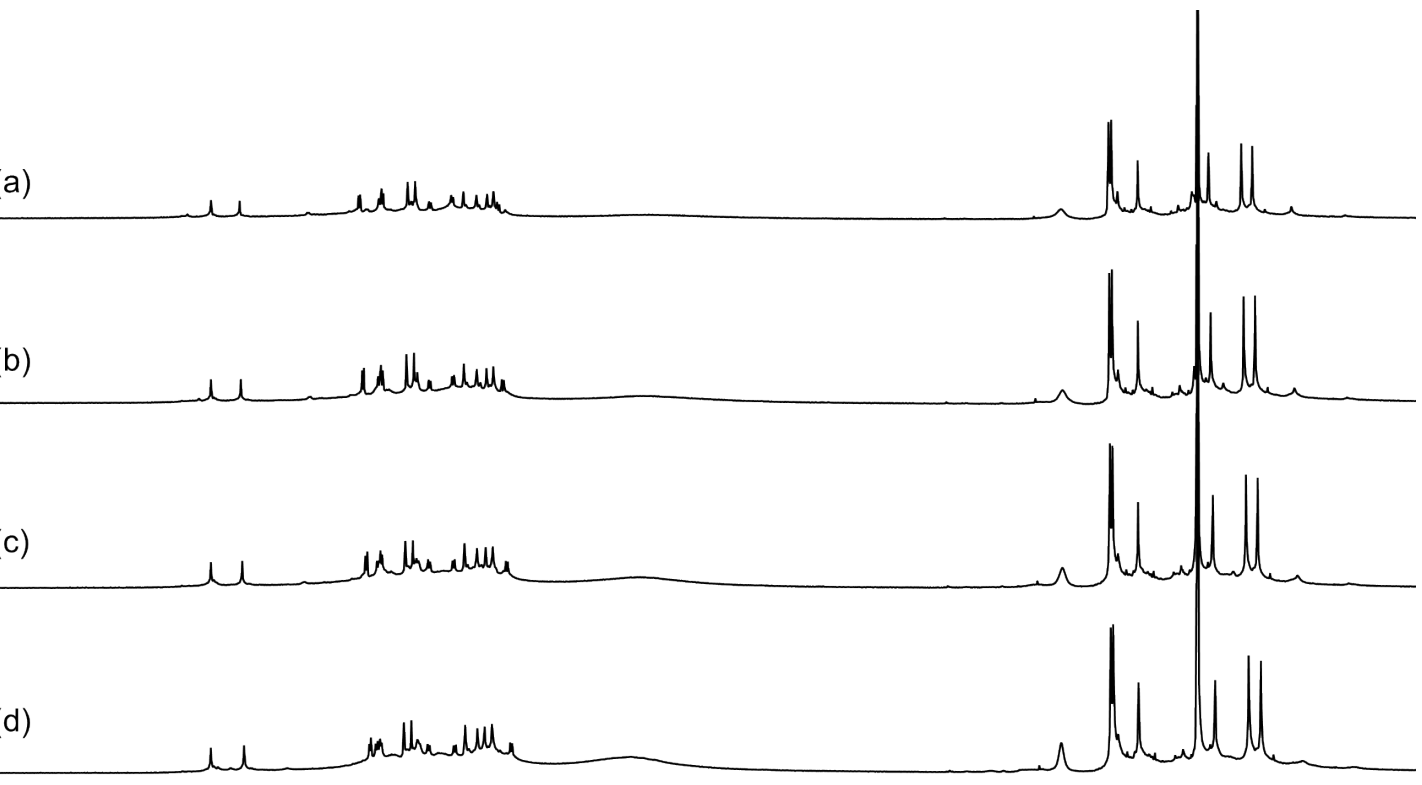

$\begin{array}{lllllllllllllllllllll}10.5 & 10.0 & 9.5 & 9.0 & 8.5 & 8.0 & 7.5 & 7.0 & 6.5 & 6.0 & \begin{array}{c}5.5 \\ \mathrm{ppm}\end{array} & 5.0 & 4.5 & 4.0 & 3.5 & 3.0 & 2.5 & 2.0 & 1.5 & 1.0 & 0.5\end{array}$

Figure S58. ${ }^{1} \mathrm{H} \mathrm{NMR}$ spectra $\left(600 \mathrm{MHz}, \mathrm{CD}_{3} \mathrm{CN}\right)$ of $\left[\mathrm{Au}_{3}(\mathbf{1 b})_{2}\right]_{2}\left(\mathrm{PF}_{6}\right)_{6}$ at different temperature, a) $313 \mathrm{~K}$, b) $293 \mathrm{~K}$, c) $273 \mathrm{~K}$, and d) $253 \mathrm{~K}$. 


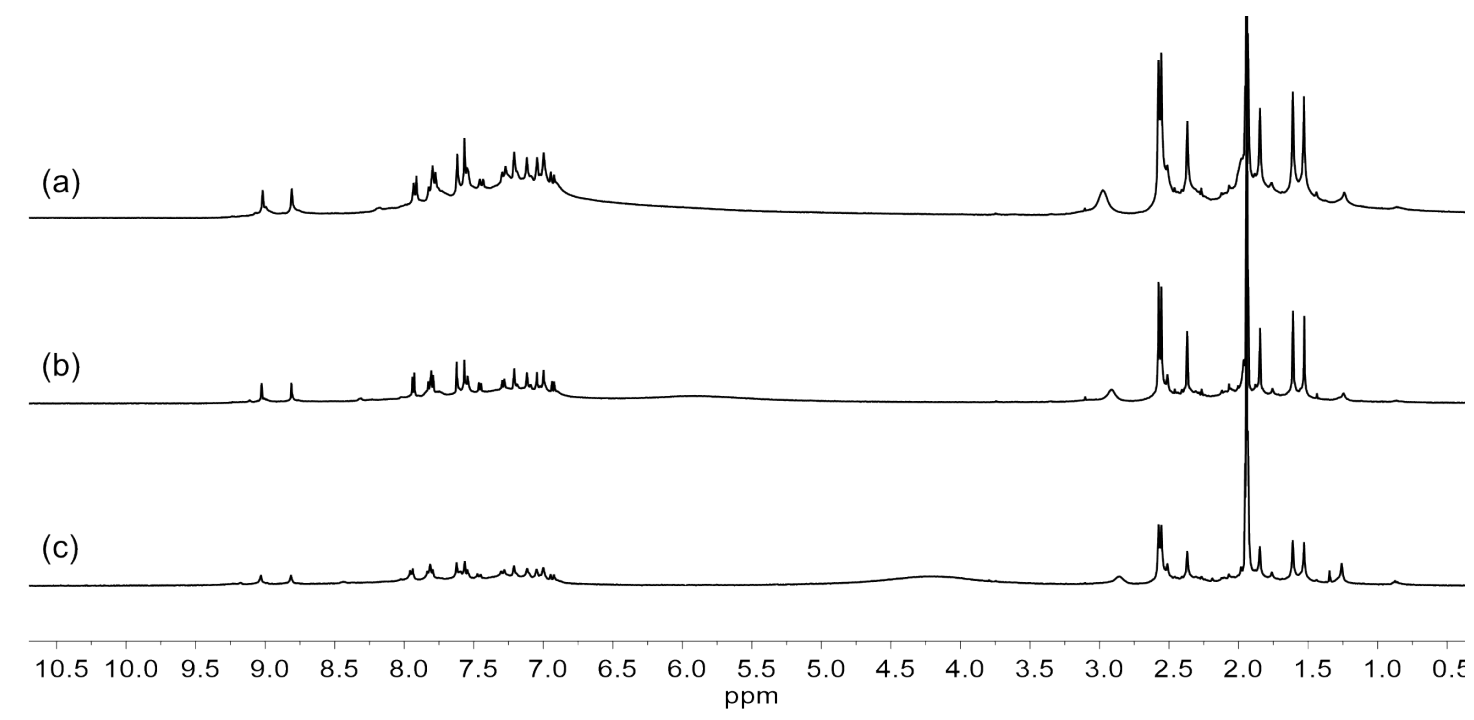

Figure S59. ${ }^{1} \mathrm{H}$ NMR spectra $\left(600 \mathrm{MHz}, \mathrm{CD}_{3} \mathrm{CN}\right)$ of $\left[\mathrm{Au}_{3}(\mathbf{1 b})_{2}\right]_{2}\left(\mathrm{PF}_{6}\right)_{6}$ at different concentrations. a) $5.5 \mathrm{mmol} / \mathrm{L}$ (nearly saturated solution), b) $3.3 \mathrm{mmol} / \mathrm{L}$, c) 1.1 $\mathrm{mmol} / \mathrm{L}$.

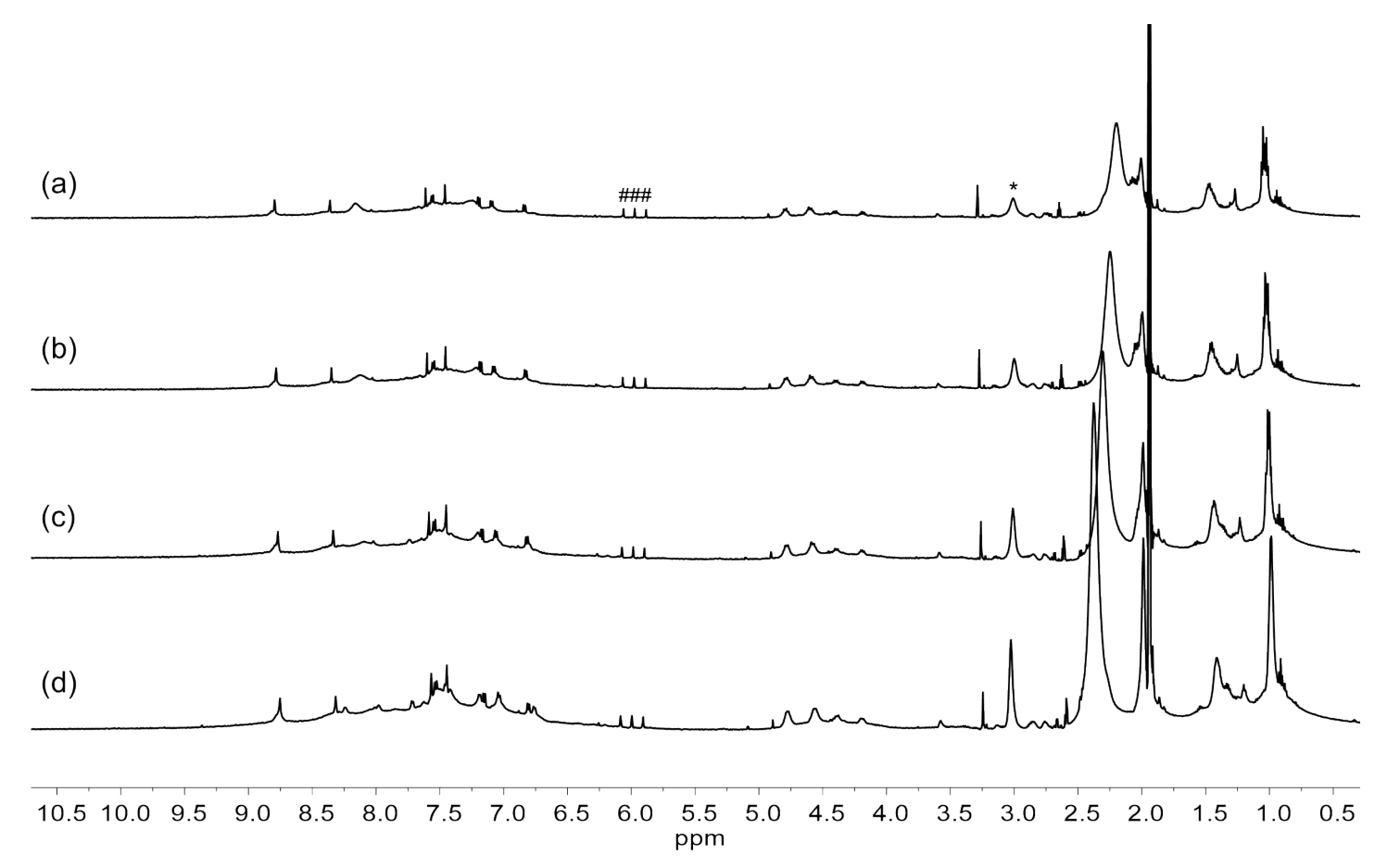

Figure S60. ${ }^{1} \mathrm{H} \mathrm{NMR}$ spectra $\left(600 \mathrm{MHz}, \mathrm{CD}_{3} \mathrm{CN}\right)$ of $\left[\mathrm{Au}_{3}(\mathbf{1 c})_{2}\right]_{2}\left(\mathrm{PF}_{6}\right)_{6}$ at different temperature, a) $313 \mathrm{~K}$, b) $293 \mathrm{~K}$, c) $273 \mathrm{~K}$, and d) $253 \mathrm{~K} .{ }^{*}$, \# = residue THT and inorganic salt. 


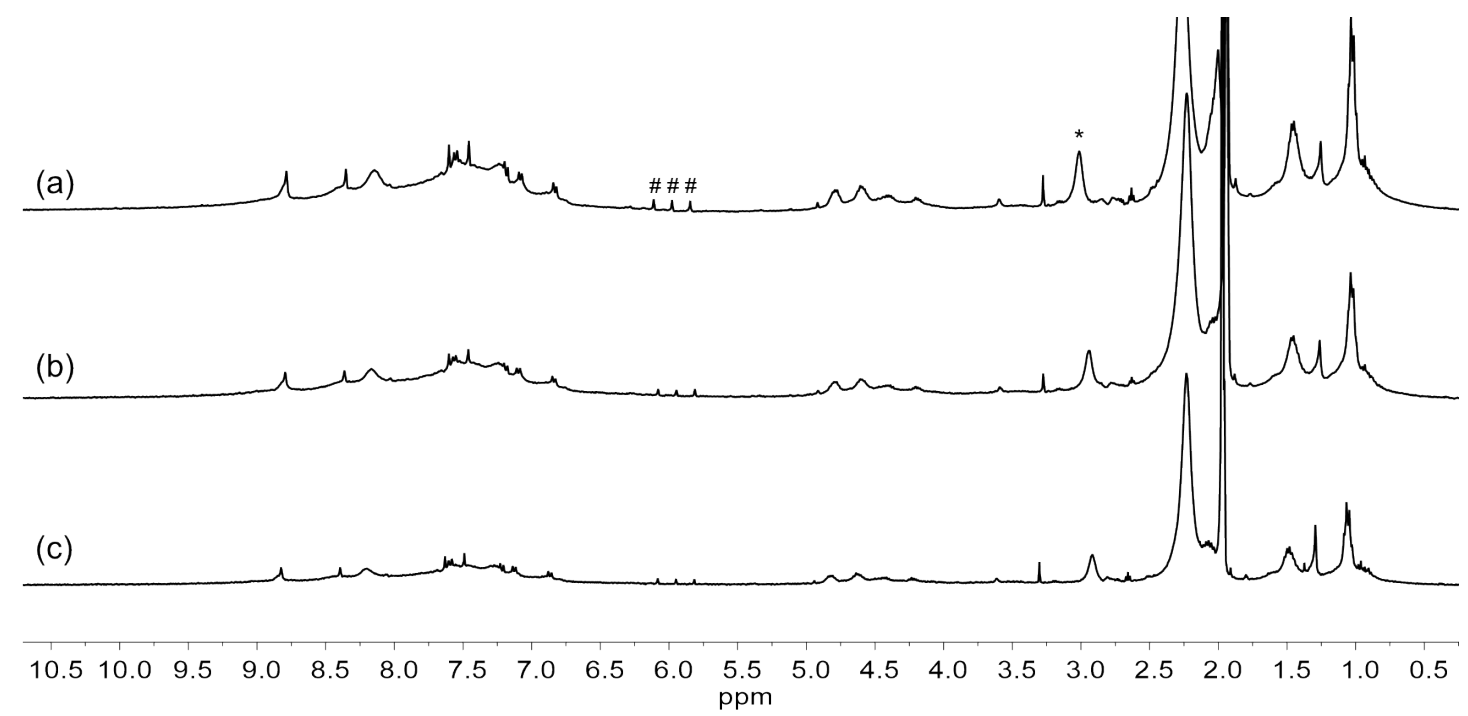

Figure S61. ${ }^{1} \mathrm{H}$ NMR spectra $\left(600 \mathrm{MHz}, \mathrm{CD}_{3} \mathrm{CN}\right)$ of $\left[\mathrm{Au}_{3}(\mathbf{1 c})_{2}\right]_{2}\left(\mathrm{PF}_{6}\right)_{6}$ at different concentrations. a) $5.5 \mathrm{mmol} / \mathrm{L}$ (nearly saturated solution), b) $3.3 \mathrm{mmol} / \mathrm{L}$, c) 1.1 mmol/L. *, \# = residue THT and inorganic salt.

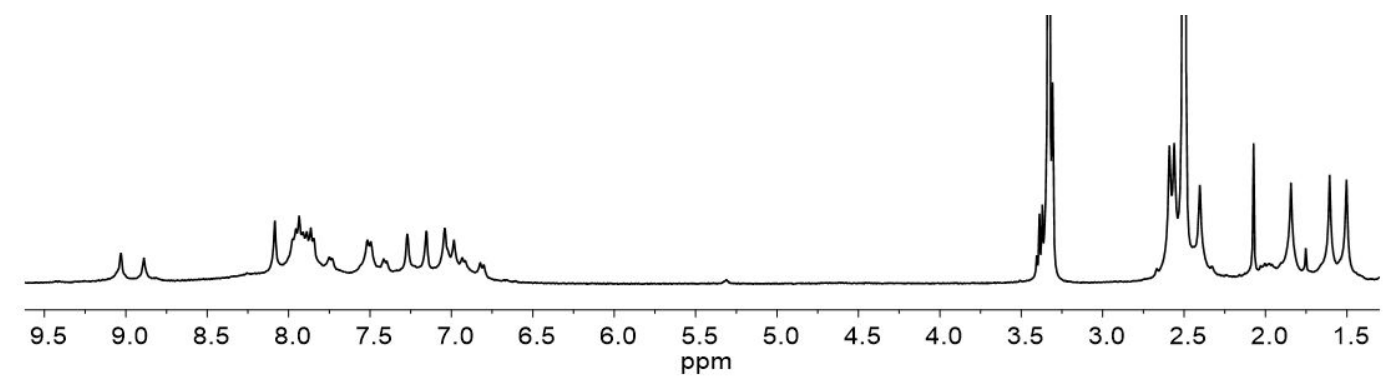

Figure S62. ${ }^{1} \mathrm{H}$ NMR spectrum $\left(400 \mathrm{MHz},\left[\mathrm{D}_{6}\right]-\mathrm{DMSO}\right)$ of $\left[\mathrm{Ag}_{3}(\mathbf{1 b})_{2}\right]_{2}\left(\mathrm{PF}_{6}\right)_{6}$.

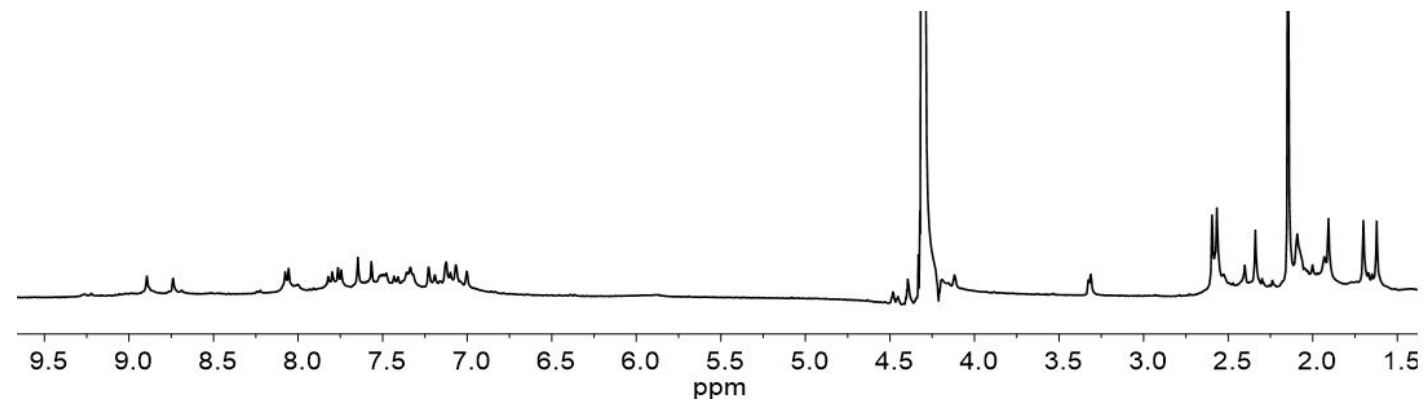

Figure S63. ${ }^{1} \mathrm{H}$ NMR spectrum $\left(400 \mathrm{MHz}, \mathrm{CD}_{3} \mathrm{NO}_{2}\right)$ of $\left[\mathrm{Ag}_{3}(\mathbf{1 b})_{2}\right]_{2}\left(\mathrm{PF}_{6}\right)_{6}$. 
(a)

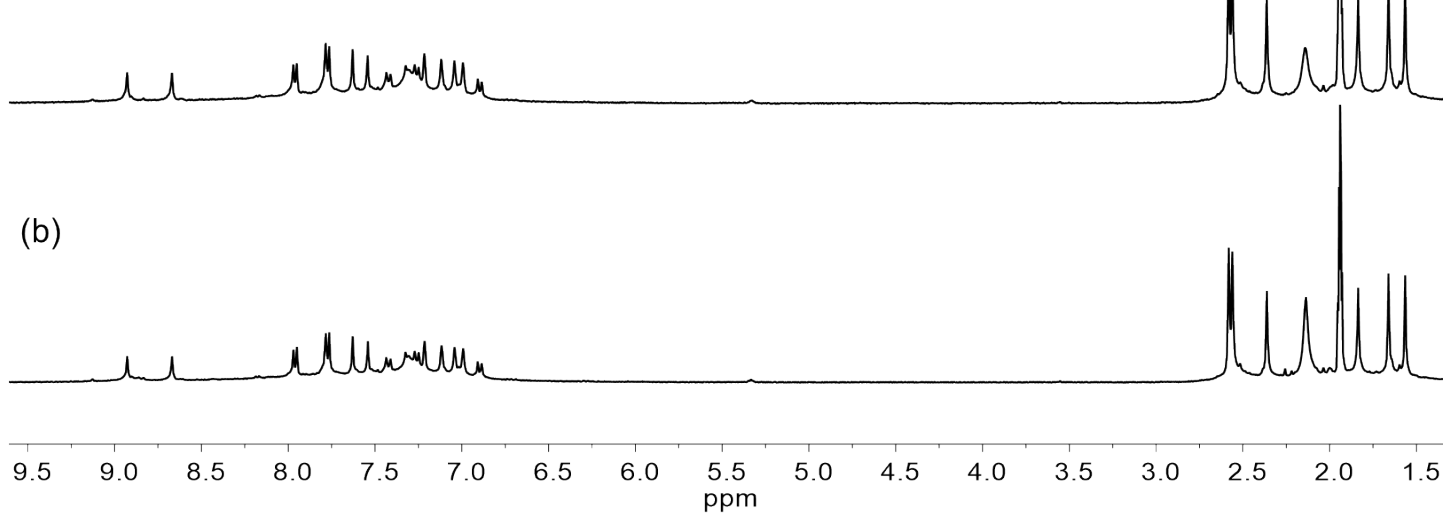

Figure S64. ${ }^{1} \mathrm{H}$ NMR spectra $\left(400 \mathrm{MHz}, \mathrm{CD}_{3} \mathrm{CN}\right)$ of $2.0 \mathrm{mM}\left[\mathrm{Ag}_{3}(\mathbf{1 b})_{2}\right]_{2}\left(\mathrm{PF}_{6}\right)_{6}$. (a) before and (b) after addition of excess coronene. (No coronene's signal was observed because it is insoluble).

(a)

(b)
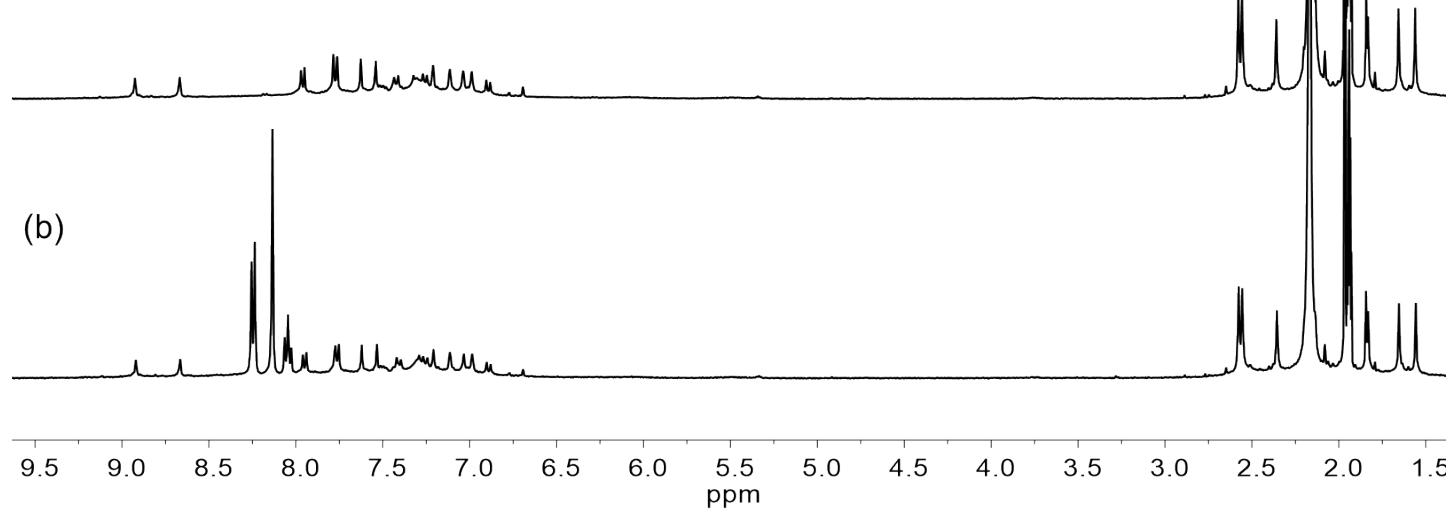

Figure S65. ${ }^{1} \mathrm{H}$ NMR spectra $\left(400 \mathrm{MHz}, \mathrm{CD}_{3} \mathrm{CN}\right)$ of $2.0 \mathrm{mM}\left[\mathrm{Ag}_{3}(\mathbf{1 b})_{2}\right]_{2}\left(\mathrm{PF}_{6}\right)_{6}$. (a) before and (b) after addition of excess pyrene. 


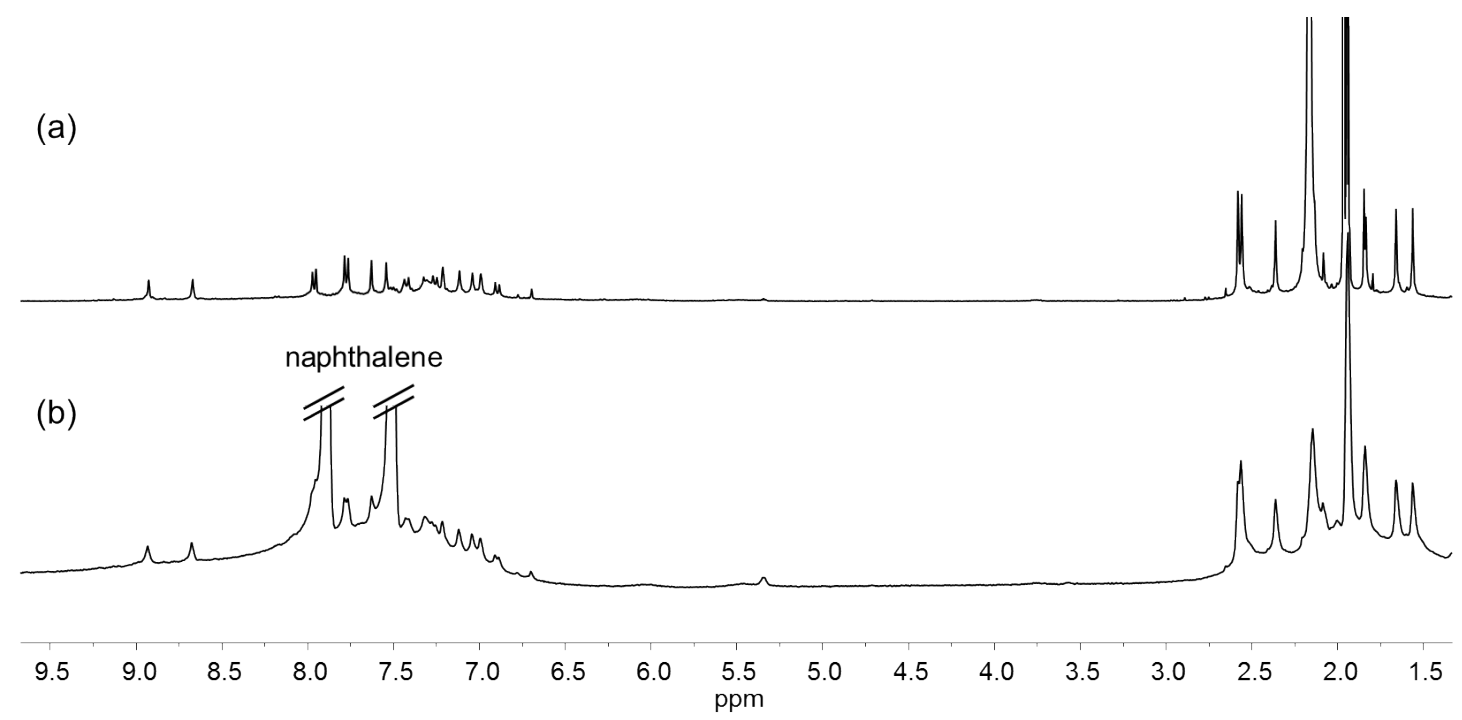

Figure S66. ${ }^{1} \mathrm{H}$ NMR spectra $\left(400 \mathrm{MHz}, \mathrm{CD}_{3} \mathrm{CN}\right)$ of $2.0 \mathrm{mM}\left[\mathrm{Ag}_{3}(\mathbf{1 b})_{2}\right]_{2}\left(\mathrm{PF}_{6}\right)_{6} \cdot(\mathrm{a})$ before and (b) after addition of excess naphthalene.

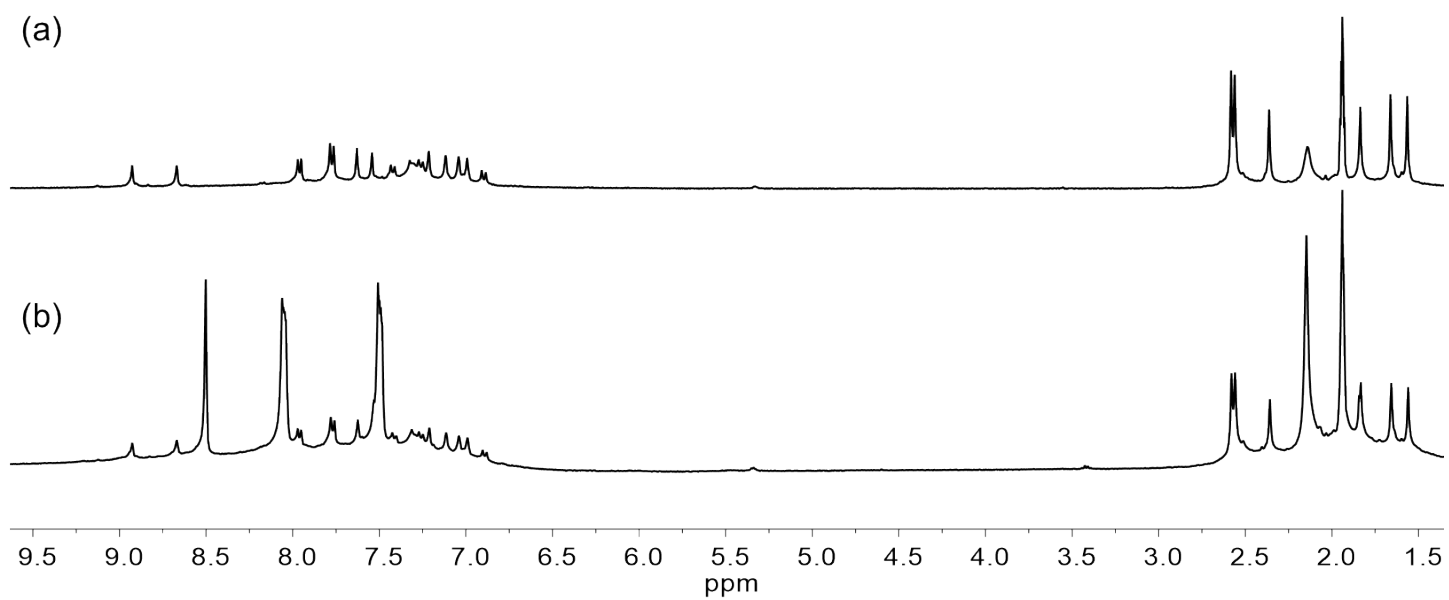

Figure S67. ${ }^{1} \mathrm{H}$ NMR spectra $\left(400 \mathrm{MHz}, \mathrm{CD}_{3} \mathrm{CN}\right)$ of $2.0 \mathrm{mM}\left[\mathrm{Ag}_{3}(\mathbf{1 b})_{2}\right]_{2}\left(\mathrm{PF}_{6}\right)_{6}$. (a) before and (b) after addition of excess anthracene. 
(a)

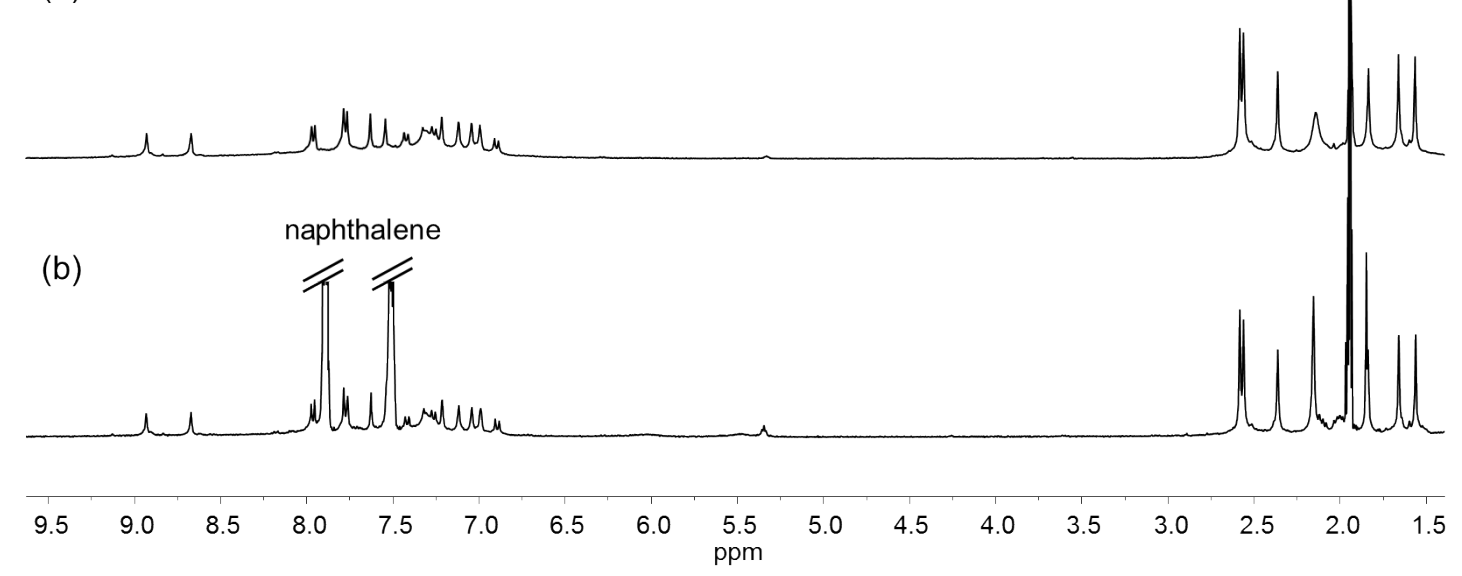

Figure S68. ${ }^{1} \mathrm{H} \mathrm{NMR}$ spectra $\left(400 \mathrm{MHz}, \mathrm{CD}_{3} \mathrm{CN}\right)$ of (a) $2.0 \mathrm{mM}\left[\mathrm{Ag}_{3}(\mathbf{1 b})_{2}\right]_{2}\left(\mathrm{PF}_{6}\right)_{6}$, (b) the product was synthesized by the addition of 10 equivalents naphthalene prior to the formation of the metal-carbene bonds under standard conditions.

(a)

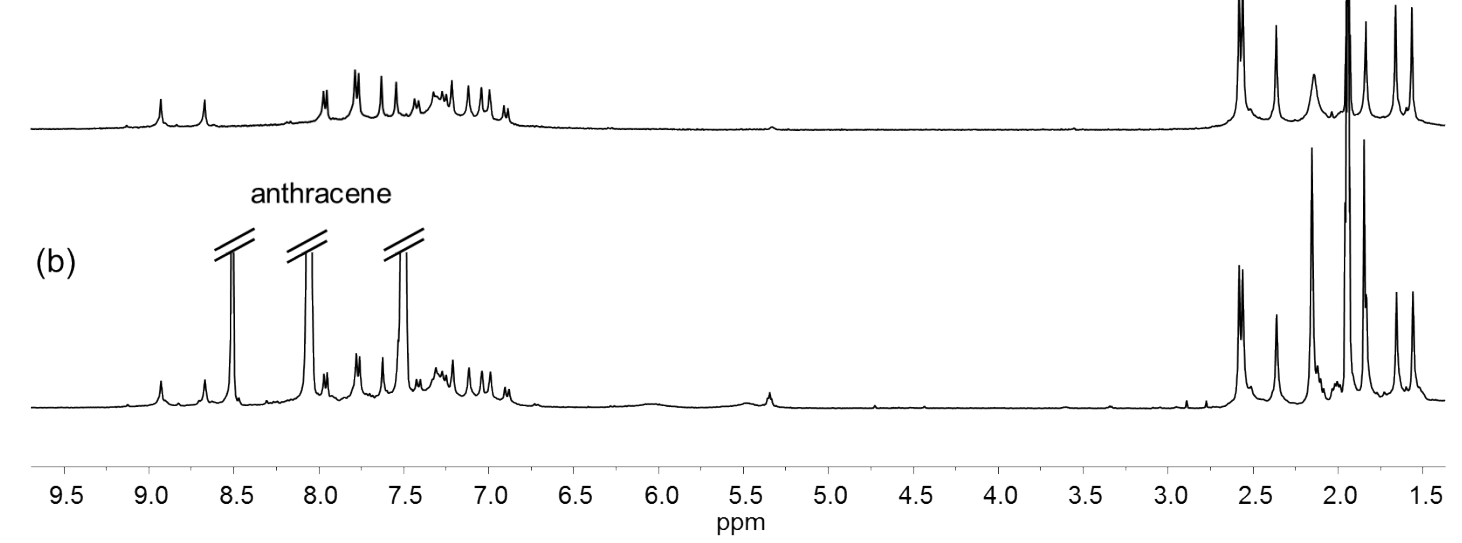

Figure S69. ${ }^{1} \mathrm{H} \mathrm{NMR}$ spectra (400 $\left.\mathrm{MHz}, \mathrm{CD}_{3} \mathrm{CN}\right)$ of (a) $2.0 \mathrm{mM}\left[\mathrm{Ag}_{3}(\mathbf{1 b})_{2}\right]_{2}\left(\mathrm{PF}_{6}\right)_{6}$, (b) the product was synthesized by the addition of 10 equivalents anthracene prior to the formation of the metal-carbene bonds under standard conditions. 
(a)

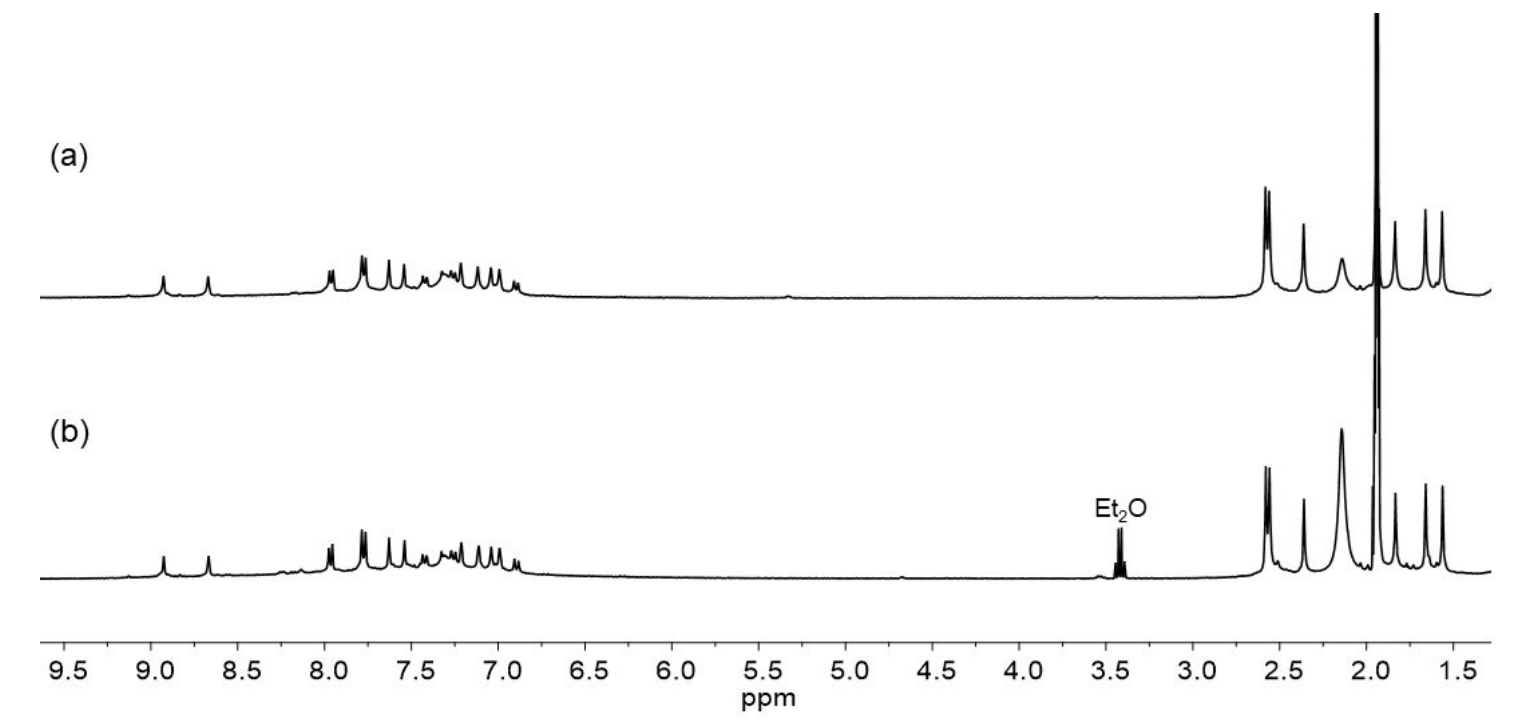

Figure S70. ${ }^{1} \mathrm{H} \mathrm{NMR}$ spectra $\left(400 \mathrm{MHz}, \mathrm{CD}_{3} \mathrm{CN}\right)$ of (a) $2.0 \mathrm{mM}\left[\mathrm{Ag}_{3}(\mathbf{1 b})_{2}\right]_{2}\left(\mathrm{PF}_{6}\right)_{6}$, (b)

the product was synthesized by the addition of 10 equivalents pyrene prior to the formation of the metal-carbene bonds under standard conditions. (Note: most of pyrene was removed after workup.) 


\section{X-ray diffraction analysis of $\left[\mathrm{Ag}_{3}(1 \mathrm{a})_{2}\right]_{2}\left(\mathrm{PF}_{6}\right)_{6}$}

\section{Crystallographic details.}

Diffraction data of $\left[\mathrm{Ag}_{3}(\mathbf{1 a})_{2}\right]_{2}\left(\mathrm{PF}_{6}\right)_{6}$ was collected with a Bruker D8 VENTURE system X-ray diffractometer at $150 \mathrm{~K}$. Raw data collection and processing were performed with APEX III software package. The data were corrected for absorption using the SADABS program. ${ }^{[\mathrm{S} 2]}$ The structure was solved with the olex2. Solve structure solution program using Charge Flipping and refined with the ShelXL refinement package using Least Squares minimisation. ${ }^{[\mathrm{S} 3, \mathrm{~S} 4]}$ The disordered solvent molecules and hexafluorophosphate anions could not be restrained properly was removed using the SQUEEZE route. The crystal data of $\left[\operatorname{Ag}_{3}(\mathbf{1 a})_{2}\right]_{2}\left(\mathrm{PF}_{6}\right)_{6}$ and selected structural parameters are summarized in Table S1. In asymmetric unit of $\left[\mathrm{Ag}_{3}(\mathbf{1 a})_{2}\right]_{2}\left(\mathrm{PF}_{6}\right)_{6}$, a Level-A alert was found due to limited resolution (1.1 $\AA$ ). Such a resolution is common in many large molecules with disordered anions and/or solvent molecules. Some Level-B alerts were found, they can be attributed to disordered moieties remained un-modelled due to the low data/parameter ratio. Given the large cell parameters and big structure, however, such is reasonable. 


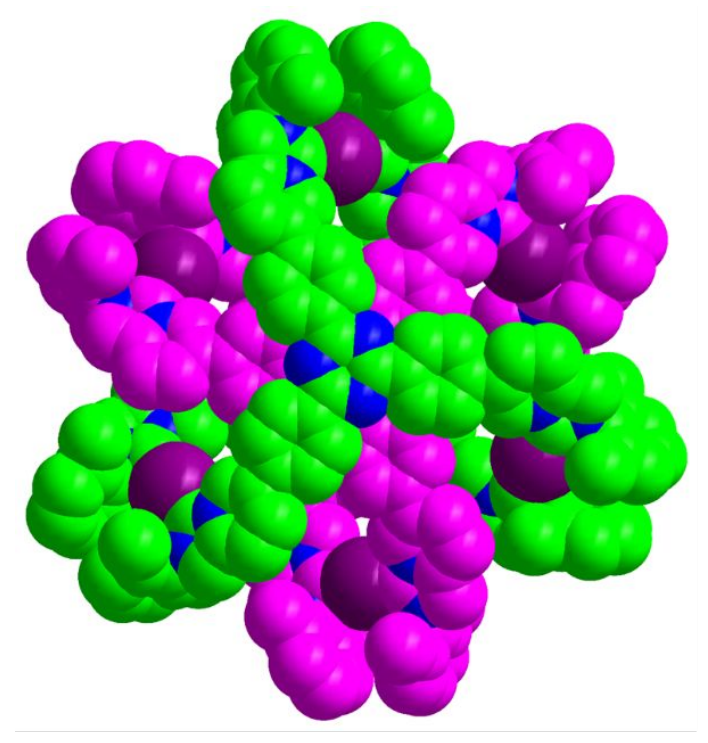

$P, \Lambda$

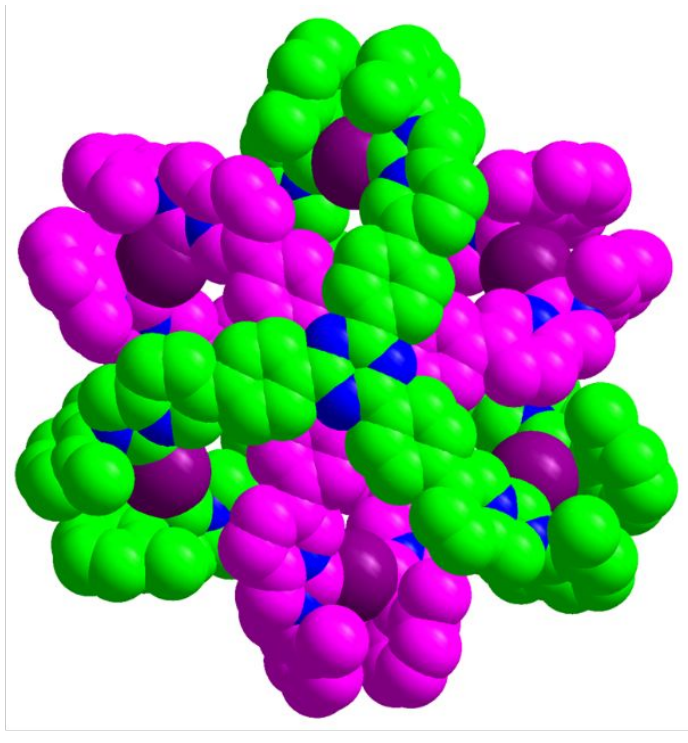

M,4

Figure S71. Van der Waals representations of the two $(P, \Lambda)$ and $(M, \triangle)$ enantiomers of $\left[\mathrm{Ag}_{3}(\mathbf{1 a})_{2}\right]_{2}\left(\mathrm{PF}_{6}\right)_{6}$. 
Table S1 Crystal data of $\left[\operatorname{Ag}_{3}\left(\mathbf{1 a}_{2}\right]_{2}\left(\mathrm{PF}_{6}\right)_{6}\right.$

\begin{tabular}{|c|c|}
\hline Identification code & 2009590 \\
\hline Empirical formula & $\mathrm{C}_{264} \mathrm{H}_{203} \mathrm{Ag}_{6} \mathrm{~F}_{18} \mathrm{~N}_{36} \mathrm{P}_{3}$ \\
\hline Formula weight & 4961.74 \\
\hline Temperature/K & 150.0 \\
\hline Crystal system & monoclinic \\
\hline Space group & $P 2_{1} / n$ \\
\hline$a / \AA ̊$ & $13.0886(14)$ \\
\hline$b / \AA$ & $48.463(5)$ \\
\hline$c / \AA$ & $52.039(6)$ \\
\hline$\alpha /^{\circ}$ & 90 \\
\hline$\beta /{ }^{\circ}$ & $95.911(5)$ \\
\hline$\gamma /{ }^{\circ}$ & 90 \\
\hline Volume $/ \AA^{3}$ & $32834(6)$ \\
\hline Z & 4 \\
\hline$\rho_{\text {calc }}\left(\mathrm{g} \cdot \mathrm{cm}^{-3}\right)$ & 1.004 \\
\hline$\mu\left(\mathrm{mm}^{-1}\right)$ & 2.270 \\
\hline$F(000)$ & 10112.0 \\
\hline Crystal size $/ \mathrm{mm}^{3}$ & $0.25 \times 0.22 \times 0.18$ \\
\hline Radiation & $\operatorname{MoK} \alpha(\lambda=1.34139)$ \\
\hline $2 \theta$ range for data collection ${ }^{\circ}$ & 3.502 to 75.376 \\
\hline Index ranges & $-11 \leq \mathrm{h} \leq 11,-41 \leq \mathrm{k} \leq 44,-47 \leq 1 \leq 47$ \\
\hline Reflections collected & 82477 \\
\hline Independent reflections & $25548\left[R_{\text {int }}=0.0823, R_{\text {sigma }}=0.0952\right]$ \\
\hline Data/restraints/parameters & $25548 / 671 / 2760$ \\
\hline Goodness-of-fit on $F^{2}$ & 1.398 \\
\hline Final $R$ indexes $[I \geq 2 \sigma(I)]$ & $\mathrm{R}_{1}=0.1226, w \mathrm{R}_{2}=0.3432$ \\
\hline Final $R$ indexes [all data] & $\mathrm{R}_{1}=0.1585, w \mathrm{R}_{2}=0.3822$ \\
\hline Largest diff. peak/hole / e $\AA^{-3}$ & $1.40 /-0.57$ \\
\hline
\end{tabular}




\section{References}

(1) Wang, Y.; Liu, W.; Deng, J.; Xie, G.; Liao, Y.; Qu, Z.; Tan, H.; Liu, Y.; Zhu, W.

Engineering the Interconnecting Position of Star - Shaped Donor- $\pi$-Acceptor Molecules Based on Triazine, Spirofluorene, and Triphenylamine Moieties for Color Tuning from Deep Blue to Green. Chem. Asian J. 2016, 11, 2555-2563.

(2) Dolomanov, O. V.; Bourhis, L. J.; Gildea, R. J.; Howard, J. A. K.; Puschmann, H. OLEX2: a Complete Structure Solution, Refinement and Analysis Program. J. Appl. Cryst. 2009, 42, 339-341.

(3) Bourhis, L. J.; Dolomanov, O. V.; Gildea, R. J.; Howard, J. A. K.; Puschmann, H. The Anatomy of a Comprehensive Constrained, Restrained Refinement Program for the Modern Computing Environment - Olex2 Dissected. Acta Cryst. 2015, A71, 5975.

(4) Sheldrick, G. M. Crystal Structure Refinement with SHELXL. Acta Cryst. 2015, C71, 3-8. 